ORNL/TM-2005/510

\title{
ASSESSMENT OF EXISTING ALLOY 617 DATA FOR GEN IV MATERIALS HANDBOOK
}

Weiju Ren and Robert W. Swindeman

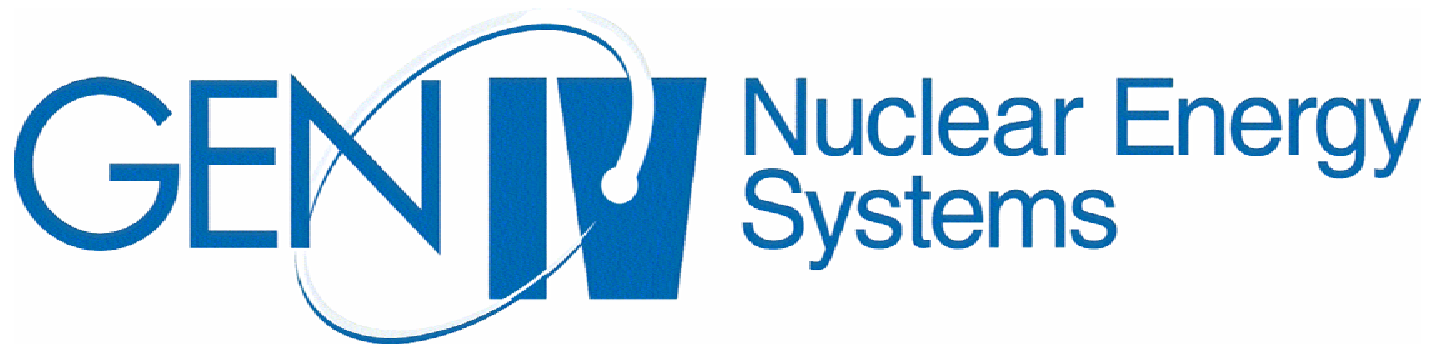

June 30, 2005 


\section{ASSESSMENT OF EXISTING ALLOY 617 DATA FOR GEN IV MATERIALS HANDBOOK}

Weiju Ren and Robert W. Swindeman

June 30, 2005

Prepared for

Office of Nuclear Energy Science and Technology

Prepared by

OAK RIDGE NATIONAL LABORATORY

Oak Ridge, Tennessee 37831 managed by

UT-BATTELLE, LLC

for the

U.S. DEPARTMENT OF ENERGY

Under DOE Contract No. DE-AC05-00OR22725 


\begin{abstract}
ABTRACT
Activities in preparing existing data on Alloy 617 for the Gen IV Materials Handbook through data mining and assessment are summarized. Status of existing data is reviewed and assessment approaches are discussed. Data classification is used to provide a reference for quality and reliability evaluation. A tracking system is developed so that all data elements can be traced back to their original source for background review whenever needed. To facilitate convenient data processing and future input into the Gen IV Materials Handbook, formats for data editing and compilation are established. Based on their priorities, existing data that are the most germane to the Gen IV nuclear reactor applications are evaluated for their data types, material status, testing conditions and other background information. Acquisition of European data on the alloy for nuclear applications is also reported.
\end{abstract}




\section{CONTENTS}

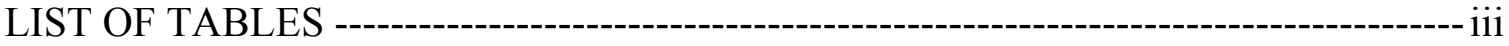

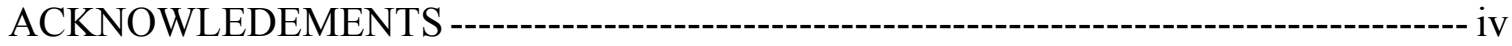

1. INTRODUCTION -- 1

1.1 Background and Motivation --- 1

1.2 Existing Data Status and Assessment Approaches ------------------------------2

2. MANAGEMENT OF ASSESSED DATA --

2.1 Classification-----------------------------------------------------------------------------------4

2.2 Tacking System-------o---on

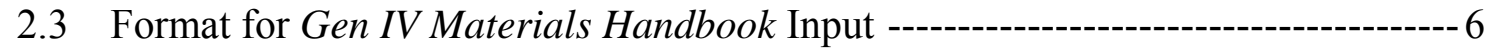

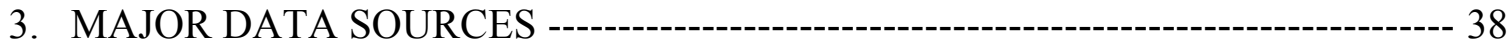

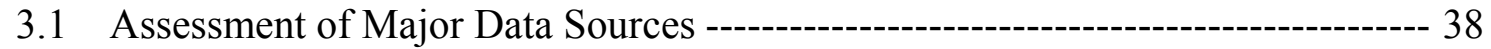

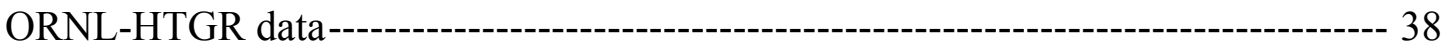

GE-HTGR data -------------------------------------------------------------------- 50

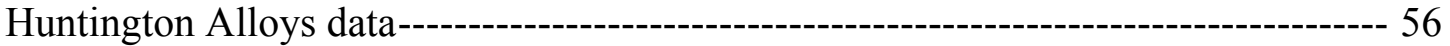

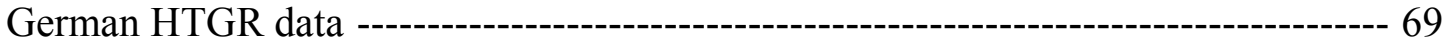

3.2 Major Data Sources in Acquisition--- 75

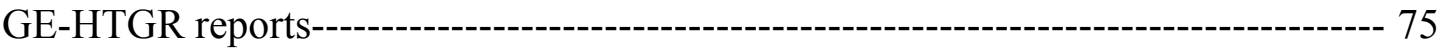

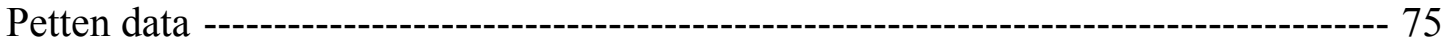

4. OTHER DATA SOURCES AND FUTURE WORK -- 79

5. REFERENCES AND DATA SOURCES --_- 80 


\section{LIST OF TABLES}

Table 1: Existing tensile property data under various testing conditions

Table 2: Existing weld tensile property data under various testing conditions

Table 3: Existing creep property data under various testing conditions

Table 4: Existing weld creep property data under various testing conditions

Table 5: Existing fatigue property data under various testing conditions

Table 6: Existing toughness property data under various testing conditions

Table 7: Number of existing tensile property data and testing conditions

Table 8: Number of existing weld tensile property data and testing conditions

Table 9: Number of existing creep property data and testing conditions

Table 10: Number of existing weld creep property data and testing conditions

Table 11: Number of existing toughness property data and testing conditions

Table 12: Summary of ORNL-HTGR data on Alloy 617

Table 13: Compositions (wt. \%) of the Alloy 617 heats used for the ORNL-HTGR data generation [McCoy285T1]

Table 14: Product characterization of the Alloy 617 heats used for the ORNL-HTGR data generation [McCoy85T2]

Table 15: Summary of GE-HTGR data on Alloy 617 (no weld data)

Table 16: Compositions (wt. \%) of the Alloy 617 heats produced for the GE-HTGR data [Baldwin486T3]

Table 17: Product characterization of the Alloy 617 heats produced by Huntington Alloy Product Division for the GE-HTGR data [Baldwin486T2]

Table 18: Helium composition in the environmental test chamber for the GE-HTGR data [Baldwin486P5]

Table 19: Estimated local gas compositions at the position of the test specimens for the GE-HTGR creep tests in simulated HTGR helium [Baldwin486]

Table 20: Summary of the Huntington Alloys data of Alloy 617

Table 21: Compositions (wt. \%) of the Alloy 617 heats for the Huntington Alloys data

Table 22: Product forms of the Alloy 617 for the Huntington Alloys data

Table 23: Chemical compositions of Alloy 617 welding electrodes used for generating the Huntington Alloys data

Table 24: Summary of the assessed German HTGR data of Alloy 617

Table 25: A summary of Mat-DB entities

Table 26: Mat-DB test result entity

Table 27: European mechanical properties data on Alloy 617 in Mat-DB 


\section{ACKNOWLEDEMENTS}

Bill Corwin, Tim McGreevy, Phil MacDonald and George Hayner participated in the review of the report. Their comments are greatly appreciated. This research is sponsored by Office of Nuclear Energy Science and Technology, U. S. Department of Energy, under contract DE-AC05-00OR22725 with UT-Battelle, LLC. 


\section{INTRODUCTION}

\subsection{Background and Motivation}

In preparing structural materials information for design and analysis of the Generation IV (Gen IV) Nuclear Reactor Systems, a considerable effort has been initiated at the Oak Ridge National Laboratory (ORNL) to develop a Gen IV Materials Handbook [Rittenhouse, 2005]. Structural materials for all the Gen IV nuclear reactor concepts supported by the Department of Energy (DOE) will be included in this Handbook. The Handbook will provide an authoritative single source of highly qualified structural materials information and offer an extensive presentation of the mechanical and physical properties data including consideration of the effects of temperature, irradiation, and environment. Necessary descriptive information such as chemical compositions and technical specifications etc. will also be incorporated for each material.

To prepare data for the Gen IV Materials Handbook, various plans and tasks have been developed covering both metallic and non metallic structural materials. Alloy 617 has been identified by the Handbook task group as well as the Gen IV Materials Program management as the first material to be collected into the Handbook through existing data mining. In "High Temperature Metallic Materials Test Plan for Generation IV Nuclear Reactors" [Ren 2004], the alloy has also been listed as the first candidate material for the data collection through material testing.

Alloy 617, also designated as Inconel 617, UNS N06617, or W. Nr. 2.4663a, was developed in the early 1970 's for high-temperature applications above $850^{\circ} \mathrm{C}\left(1562^{\circ} \mathrm{F}\right)$. It is a nickel-chromium-cobalt-molybdenum alloy with a good combination of hightemperature strength and oxidation resistance. The alloy also has excellent resistance to a wide range of corrosive environments, and it is readily formed and welded by conventional techniques. Since its development, its properties have made it an attractive material and often considered for use in aircraft and land-based gas turbines, chemical manufacturing components, metallurgical processing facilities, and fossil and nuclear power generation structures. Significant amount of data on the alloy has been generated domestically and internationally over the past more than 30 years for these applications. This existing data provides a wealth of information sources for the Gen IV Materials Handbook.

However, because the existing data were produced by different domestic and international sources, they may have been generated under different standards, using different testing techniques, through different data reduction procedures, and for different application purposes. Before these data can be accepted into the Gen IV Materials Handbook, their quality, suitability, testing conditions and other background information must be carefully collected, reviewed, classified, edited, compiled, and even verified by experiments when necessary. All this has necessitated the task of existing data assessment. 
On the other hand, because the thermal, environmental, and service life conditions of the Gen IV nuclear reactors are unprecedented with respect to previous application experiences of the alloy, a large database is required to evaluate the alloy's viability and qualification for the intended service conditions. For example, in the leading Gen IV nuclear reactor candidate - the Very High Temperature Reactor (VHTR), helium coolant at an outlet temperature of $900 \sim 1000^{\circ} \mathrm{C}\left(1652 \sim 1932^{\circ} \mathrm{F}\right)$ and a design life of up to 60 years are required. Material properties data for some of such conditions may not exist, and must be generated through experiments under simulated reactor working conditions. Generating data under such severe testing conditions entails a considerable amount of cost, time and manpower. Needless to say, an assessment of the existing data can help efficiently identify the data gaps (data-unavailable) and pinpoint experimental and testing needs, and thus significantly reduce cost, save time, and minimize manpower in data generation. Therefore, in the "High Temperature Metallic Materials Test Plan for Generation IV Nuclear Reactors" [Ren 2004], assessment of existing data has been identified as one of the very important early steps in the Gen IV materials testing program.

\subsection{Existing Data Status and Assessment Approaches}

Information on Alloy 617 can be found in many sources including ASME and ASTM documents, recognized international codes and standards, various materials handbooks, manufacturer records as well as open literature. Early in this data assessment effort, sources containing information on Alloy 617 were searched through the CSA Materials Research Database with METADEX. A title search has yielded more than 100 documents; and a key word search has resulted in more than 700. Unpublished/internal documents have also been identified and located through conventional searching methods such as file digging and personal communications. As a matter of fact, much information germane to the Gen IV nuclear reactor applications on the alloy has been found in unpublished/internal documents, for example: the reports on investigation of Alloy 617 for DOE's High Temperature Gas-Cooled Reactors (HTGR) Program generated in the 1980's.

The data assessment process has included collecting, assembling, reviewing, analyzing, accepting or rejecting, compiling, and documenting the existing data. To manage and process such a large quantity of information, an early preliminary review was conducted to evaluate the status of the existing data and determine the assessment approaches. It was decided that to ensure efficiency of the entire assessment process, the identified documents must be evaluated on a priority basis. Therefore, documents that apparently contain data relevant and important to the Gen IV nuclear reactor service conditions have been evaluated first. Those with relatively lower relevancy to the Gen IV materials data needs have been left for future review due to the limited time frame given to deliver the present report. Some documents have been excluded from the assessment after the preliminary review indicated that the information was of little use or not germane to the Gen IV materials data needs. It has also been realized in the preliminary review that although a lot of documents exist, many of them contain data that 


\section{ASSESSMENT OF EXISTING ALLOY 617 DATA FOR GEN IV NUCLEAR REACTOR APPLICATIONS}

are either duplicated or processed from data in other documents. For example, some ASME Code Cases and the draft Code Case for the design of Alloy 617 nuclear components contain data duplicated and/or processed from data in some other documents. There are also sources that have been identified to contain information of great interest to the Gen IV materials data needs, but have been unable to be evaluated for the present report due to availability limitations from factors such as proprietary rights and commercial interests. Communication and negotiations have been conducted and will be continued to gain access to those sources.

The early preliminary review has also revealed that most of the existing data are presented in various hard copy forms such as tables, figures and texts. To prepare these data for future input into the Gen IV Materials Handbook as well as the analyses during the assessment, appropriate formats for convenient data maneuvering should first be developed, and then the hard copy data must be digitized into electronic form and converted into the developed formats for future use. Moreover, to facilitate data analyses and future applications, a tracking system is also needed so that every data element, i. e., point, micrograph, quoted text statement etc., can be traced back to its original source when desired. To help evaluate the data quality and relevancy to the Gen IV nuclear reactor applications, classification criteria are also needed to categorize the existing data. To assist in conveniently identifying data gaps, the digitized and formatted data should be used to extract their testing conditions and matrices from which they have been generated.

The present report summarizes the efforts and progress in the collection and evaluation of the existing data on Alloy 617 over the period from October 2004 to June 2005. There is no doubt that new data are being generated somewhere around the world while the present assessment is in progress. Therefore, although the present report may have collected existing data on Alloy 617 most germane to the Gen IV nuclear reactors, it should not be considered a comprehensive and conclusive assessment. Efforts will be continued to collect and evaluate useful existing data on the alloy for the Gen IV materials program. 


\section{MANAGEMENT OF EXISTING DATA}

\subsection{Classification}

Because the existing data are collected from various sources, decisions must be constantly made about their quality, reliability, relevancy, and acceptability. Without specific guidelines, these decisions will largely be left to personal judgment of the individuals who are processing the data. In this case, conflicts and inconsistencies would become inevitable. Therefore, it is necessary that some commonly agreed guidelines are provided for making such judgment. However, existing data from various sources may have been generated under different testing standards, processed with different procedures, and produced with different error allowances. It is very difficult to establish an absolute standard that can be used for judging their relative quality. Since the assessed data will eventually be input into the Gen IV Materials Handbook, the criteria for classification and identification proposed for the Gen IV Materials Handbook will be followed. Based mainly on the origin of the sources, the existing data will be categorized into five classes as follows [Rittenhouse 2005]:

Class 1 These materials data meet all DOE Gen IV Reactor Programs and NRC QA requirements (i.e., these are data generated in documented $\mathrm{R} \& \mathrm{D}$ programs that meet all of the requirements of 10CFR50 Appendix B and DOE/NRC agreed versions of NQA-1). It is expected that the new data generated in Gen IV materials programs will be of this category. Data with this pedigree, it is assumed, would be entirely acceptable to DOE, NRC, and reactor vendors for use in final design and design analyses, especially if they are submitted to and approved by appropriate codes and standards bodies.

Class 2 Materials data and data correlations provided in various sections of wellrecognized U.S. codes and standards (e.g., ASME and ASTM) will be designated as Class 2. In many cases the raw data (i.e., individual data points) will not be available from these sources. Thus, the results of peerapproved analyses and resulting data correlations contained in these codes and standards may be the major Class 2 input to the Handbook. Further, although materials and materials data approved by codes and standards bodies are generally a necessary condition for acceptance of designs by State and Federal regulatory bodies, there may in some cases be additional requirements.

Class 3 Materials data provided in well-recognized international codes and standards will be categorized at present as Class 3. This may be revisited as the result of any international agreements reached relative to cooperation on the Handbook. For example, such agreements might result in Classes 2 and 3 being combined into a single class. 
Class 4 Materials data obtained from materials handbooks such as the Nuclear Systems Materials Handbook and the AFCI Materials Handbook will be identified as Class 4. The data contained in these two examples have had careful and extensive analysis and peer review (equivalent to Class 2) but the data were generated under quality conditions ranging from "unknown" to equivalent to Class 1. In the latter case, the data from such handbooks would be listed as Class 1 .

Class 5 Materials data obtained from sources such as manufacturers brochures and the open literature will be categorized as Class 5. Such data will, of course, be reviewed and approved before it is incorporated into the Handbook.

Although the classifications described above are not an absolute measure of the quality of existing data, they do provide a common basis for guidance on data acceptability and assurance. Further, questions relative to data uncertainties and ranges will be addressed in the Gen IV Materials Handbook by providing individual data points (whenever possible), including a characterization of each data point in terms of material and test parameters, source, and incorporating software packages for statistical analysis. An additional consideration for an attribute to determine data quality beyond test results and test condition is test technique. How the test was conducted can be a major indicator of the quality of the resulting data. If an existing data set appears obviously questionable, testing may also be conducted to verify the reliability before the data are rejected or accepted.

\subsection{Tracking System}

Because the existing data were generated by various sources, their detailed background information such as the testing conditions, standards, intended applications, data processing methods etcetera may vary from one another. In the present assessment and even when the data are used for the analysis and design of the Gen IV nuclear reactor components, such information may become very important for understanding the material behavior exhibited by the data, especially when questions arise about their background. Therefore, the origin of the data should not be lost or clouded when data points are assembled, edited, formatted, and compiled for the Handbook input or when they are used for design and analysis after they have been stored in the Handbook. It is apparent that a tracking system must be established and applied to all the data elements early in the assessment stage. When the data are installed into the Handbook, a user should be able to easily track down the origin of each data element. Because so many data elements need to be tracked, the system must be kept very simple but reasonably accurate. An efficient way to do this is to attach a short ID tag to each data element.

To clearly indicate the origin of a data element with a short ID tag, the tag must include the identification of the source, usually a document or an established electronic database that has originally contained the data element. The shortest identification for a source document in most cases is the last name of the author plus date of publication. For 
some corporate documents without an author identified, an acronym composed of the first letters of the company name, or its well known nickname may be used. The same method can be applied to tag data elements from established electronic databases. To keep the publication date short, only the month in numeric number and the last two digits of the year should be used. Furthermore, since in most cases the source document is large, the location of the data element in the source document should be identifiable from the tag. The location identification may not be that crucial for data elements from an established electronic database since electronic search functions are always available for such databases and electronic databases are usually dynamic and subject to changes. For hard copy source documents, the data element location can be indicated with Tx for Table x, Fy for Figure y, and Pz for page z. The tag for a data point of creep rupture stress from Table 4 of a document authored by John Doe published in July, 1978, for example, can be created as "Doe0778T4". Whenever the data point is listed in a compiled new table in the assessment, a Source Column is always dedicated for the tag. If the data element is a figure, the tag should be attached to the end of the caption; and for a text statement, the tag goes after the sentence. When the Handbook is developed, the tag may be used as a hypertext link to the source document if the document is installed in the electronic Handbook database. An easy click of finger on the tag should bring up the source document and display all the available background information for the user to review.

\subsection{Formats for Gen IV Materials Handbook Input}

Because the main purpose of the present assessment is to prepare qualified existing data for the Gen IV Materials Handbook, which will be constructed as a web-accessible electronic database with analytical and processing power, the assessed data should be edited and compiled into electronic files, and appropriate formats must be developed for data storage and presentation. The data that are originally presented in forms of numerical numbers or plots in hard copy source documents must first be converted into digitized electronic numbers that can be mathematically operated by computer.

The use of Microsoft Excel spread sheets has been selected for storing the evaluated data for future input into the Gen IV Materials Handbook. The spread sheet format provides a great advantage of being able to add multiple columns as needed without being limited by the width of document paper or computer screen. Its "Freeze Panes" function also allows the column and row titles to remain visible during scrolling along multiple columns and rows. Most importantly, because spread sheet is designed for data processing, many functions needed for data manipulation are available, and these functions may mostly be required by the Gen IV Materials Handbook.

For future convenient data input into the Handbook, the evaluated data should be categorized based on the types of property in the same manner as will be categorized in the Handbook [Rittenhouse 2005]. Therefore, appropriate data presentation formats have been developed for each type of property. Examples of the formats developed for tensile properties, creep properties, and toughness properties are shown as Table 1 for base metal 
tensile properties, Table 2 for weld tensile properties, Table 3 for base metal creep properties, Table 4 for weld creep properties, Table 5 for fatigue properties, and Table 6 for toughness properties, respectively. The specimen material conditions such as heat treatment and aging parameters are intentionally separated into columns of unaged treatment, aging time, aging temperature etc. so that the data can be conveniently sorted based on these parameters by using the "Sort" function of the spread sheet. Due to the limited page width of the present report, rows of the example tables are continuously presented on consecutive pages. Alphabet letters are used to indicate continuation of the rows on different pages. It should be stressed that the alphabet letters will neither be needed nor present in the full electronic version of the formatted data because the electronic tables can be continuously scrolled regardless of the width of computer screen. The explanations for acronyms and symbols in the examples will not present in the electronic Handbook in the fashion shown here but may be installed in a drop down dialogue window. User can click on the button to review them whenever he/she desires. The full version of the formatted data is not presented in this report partly due to its large size and electronic form. More importantly it is because converting hard copy numbers, plot points and curves into electronic data is a tedious, time consuming task that requires great care and accuracy, all the converted and formatted data must be double checked for error. Further, more existing data sources are still being obtained, and the spread sheets with formatted electronic data are subjected to continuous changes and expansions. Therefore, before the Gen IV Materials Handbook is ready to accept data input, release of the assessed, formatted existing data should be limited to avoid unnecessary multiversion confusion in the future.

To facilitate identifying the data gaps, the evaluated data tables have been further processed to extract the testing conditions under which the existing data were generated. The results of the extraction can be formatted into test matrices that were used to generate the existing data; and the conditions under which the material has not been tested can then be identified by comparing the test matrices with the data needs for analysis and design. Examples of the extracted test conditions and numbers of the existing data for each condition are given in Table 7 for tensile tests on base metal, Table 8 for tensile tests on weld metal, Table 9 for creep tests on base metal, Table 10 for creep tests on weld metal, and Table 11 for toughness tests on base metal. The extraction of testing conditions presented in the present report is only a preparation exercise for such practice. When the web-accessible Gen IV Materials Handbook is constructed, extraction of testing conditions for test matrices will be required as one of the important functionalities of the Handbook. With a click of finger, dedicated servers in the Handbook Application Tier will quickly browse through the entire database, extract the testing conditions of existing data, convert the conditions into test matrices that were used to generate the existing data, compare them with the data needs for analysis and design, and recommend new test matrices for generating new data to fill data gaps. 
Table 1: Existing tensile property data under various testing conditions

\begin{tabular}{|c|c|c|c|c|c|c|c|}
\hline Heat & Form & $\begin{array}{c}\text { Test } \\
\text { ID }\end{array}$ & $\begin{array}{c}\text { T-Test } \\
{ }^{\circ} \mathrm{C}\end{array}$ & $\begin{array}{c}\text { UnAged } \\
\text { Treat }\end{array}$ & AgeEnv & $\begin{array}{c}\text { T-Age } \\
{ }^{\circ} \mathrm{C}\end{array}$ & $\bar{a}$ \\
\hline XX01A3US & $13 \mathrm{~mm}$ plate & 16545 & 22 & $\begin{array}{l}\text { solution } \\
\text { annealed }\end{array}$ & N/A & N/A & $\mathrm{b}$ \\
\hline XX01A3US & $13 \mathrm{~mm}$ plate & 16546 & 22 & $\begin{array}{c}\text { solution } \\
\text { annealed }\end{array}$ & $\mathrm{N} / \mathrm{A}$ & $\mathrm{N} / \mathrm{A}$ & $\mathrm{c}$ \\
\hline XX01A3US & $13 \mathrm{~mm}$ plate & 16547 & 22 & $\begin{array}{l}\text { solution } \\
\text { annealed }\end{array}$ & N/A & N/A & $\mathrm{d}$ \\
\hline XX01A3US & $13 \mathrm{~mm}$ plate & 17897 & 22 & $\mathrm{~N} / \mathrm{A}$ & inert & 538 & $\mathrm{e}$ \\
\hline XX01A3US & $13 \mathrm{~mm}$ plate & 17898 & 22 & $\mathrm{~N} / \mathrm{A}$ & inert & 538 & $\mathrm{f}$ \\
\hline XX01A3US & $13 \mathrm{~mm}$ plate & 17905 & 22 & N/A & inert & 871 & $\mathrm{~g}$ \\
\hline XX01A3US & $13 \mathrm{~mm}$ plate & 17906 & 22 & N/A & inert & 871 & $\mathrm{~h}$ \\
\hline XX01A3US & $13 \mathrm{~mm}$ plate & IC43 & 22 & $\mathrm{~N} / \mathrm{A}$ & inert & 538 & $\mathrm{i}$ \\
\hline XX01A3US & $13 \mathrm{~mm}$ plate & 16657 & 538 & $\begin{array}{c}\text { solution } \\
\text { annealed }\end{array}$ & $\mathrm{N} / \mathrm{A}$ & N/A & $\mathrm{j}$ \\
\hline XX01A3US & $13 \mathrm{~mm}$ plate & 17899 & 538 & $\mathrm{~N} / \mathrm{A}$ & inert & 538 & $\mathrm{k}$ \\
\hline XX01A3US & $13 \mathrm{~mm}$ plate & 16664 & 704 & $\begin{array}{c}\text { solution } \\
\text { annealed }\end{array}$ & N/A & N/A & 1 \\
\hline XX01A3US & $13 \mathrm{~mm}$ plate & 16665 & 704 & $\begin{array}{c}\text { solution } \\
\text { annealed }\end{array}$ & $\mathrm{N} / \mathrm{A}$ & $\mathrm{N} / \mathrm{A}$ & $\mathrm{m}$ \\
\hline XX01A3US & $13 \mathrm{~mm}$ plate & 17903 & 704 & N/A & inert & 704 & $\mathrm{n}$ \\
\hline XX01A3US & $13 \mathrm{~mm}$ plate & 179040 & 704 & N/A & inert & 704 & o \\
\hline XX01A3US & $13 \mathrm{~mm}$ plate & IC27 & 704 & $\mathrm{~N} / \mathrm{A}$ & inert & 704 & $\mathrm{p}$ \\
\hline XX01A3US & $13 \mathrm{~mm}$ plate & IC8 & 871 & N/A & inert & 871 & $q$ \\
\hline XX01A3US & $13 \mathrm{~mm}$ plate & $\mathrm{I}-105$ & 704 & $\mathrm{~N} / \mathrm{A}$ & impure $\mathrm{He}$ & 704 & $\mathrm{r}$ \\
\hline XX01A3US & $13 \mathrm{~mm}$ plate & $\mathrm{I}-120$ & 871 & $\mathrm{~N} / \mathrm{A}$ & impure $\mathrm{He}$ & 871 & $\mathrm{~S}$ \\
\hline XX09A4UK & $9.5 \mathrm{~mm}$ plate & 203 & 704 & unknown & $\mathrm{N} / \mathrm{A}$ & $\mathrm{N} / \mathrm{A}$ & $\mathrm{t}$ \\
\hline XX09A4UK & $9.5 \mathrm{~mm}$ plate & 204 & 760 & unknown & $\mathrm{N} / \mathrm{A}$ & $\mathrm{N} / \mathrm{A}$ & $\mathrm{u}$ \\
\hline XX01A3US & $13 \mathrm{~mm}$ plate & - & 25 & N/A & inert & 482 & $\mathrm{v}$ \\
\hline$\downarrow$ & $\downarrow$ & $\downarrow$ & $\downarrow$ & $\downarrow$ & $\downarrow$ & $\downarrow$ & $\downarrow$ \\
\hline
\end{tabular}

Heat $=$ heat identification

Form $=$ product form

Test ID $=$ identification of the test that generated the data

T-Test $=$ test temperature

UnAged Treat $=$ heat treatment for sample without aging

AgeEnv = aging environment for aged sample

T-Age $=$ aging temperature 
ASSESSMENT OF EXISTING ALLOY 617 DATA FOR GEN IV NUCLEAR REACTOR APPLICATIONS

Table 1 (cont'd): Existing tensile property data under various testing conditions

\begin{tabular}{|c|c|c|c|c|c|c|c|}
\hline $\bar{a}$ & $\begin{array}{c}\text { t-Age } \\
\text { h }\end{array}$ & $\begin{array}{c}0.2 \% Y S \\
\text { MPa }\end{array}$ & $\begin{array}{l}\text { UTS } \\
\text { MPa }\end{array}$ & $\begin{array}{c}\text { UnifStn } \\
\%\end{array}$ & $\begin{array}{c}\text { TotalStn } \\
\%\end{array}$ & $\begin{array}{c}\text { RA } \\
\%\end{array}$ & $\overline{\mathbf{a}}$ \\
\hline $\mathrm{b}$ & 0 & 303 & 747 & 54.1 & 54.3 & 36.2 & $\mathrm{~b}$ \\
\hline $\mathrm{c}$ & 0 & 301 & 757 & 59.6 & 60.9 & 40.7 & $\mathrm{c}$ \\
\hline $\mathrm{d}$ & 0 & 305 & 754 & 57.4 & 57.9 & 32.4 & $\mathrm{~d}$ \\
\hline $\mathrm{e}$ & 2,500 & 358 & 794 & 66.0 & 68.1 & 49.3 & $\mathrm{e}$ \\
\hline $\mathrm{f}$ & 2,500 & 360 & 796 & 67.6 & 69.8 & 48.6 & $\mathrm{f}$ \\
\hline $\mathrm{g}$ & 2,500 & 331 & 797 & 29.9 & 29.9 & 25.0 & $\mathrm{~g}$ \\
\hline $\mathrm{h}$ & 2,500 & 334 & 822 & 34.6 & 34.6 & 29.3 & $\mathrm{~h}$ \\
\hline $\mathrm{i}$ & 10,000 & 392 & 805 & 53.3 & 53.4 & 43.8 & $\mathrm{i}$ \\
\hline $\mathrm{j}$ & 0 & 216 & 610 & 64.7 & 67.3 & 49.8 & $\mathrm{j}$ \\
\hline $\mathrm{k}$ & 2,500 & 260 & 638 & 63.4 & 68.5 & 51.7 & $\mathrm{k}$ \\
\hline 1 & 0 & 199 & 466 & 29.3 & 68.0 & 49.3 & 1 \\
\hline $\mathrm{m}$ & 0 & 196 & 443 & 25.9 & 69.9 & 53.1 & $\mathrm{~m}$ \\
\hline $\mathrm{n}$ & 2,500 & 257 & 502 & 16.2 & 75.3 & 57.6 & $\mathrm{n}$ \\
\hline o & 2,500 & 256 & 475 & 14.4 & - & - & $\mathrm{o}$ \\
\hline $\mathrm{p}$ & 10,000 & 211 & 461 & 16.9 & 68.8 & 56.3 & $\mathrm{p}$ \\
\hline$q$ & 10,000 & 170 & 181 & 8.0 & 90.9 & 79.2 & $\mathrm{q}$ \\
\hline $\mathrm{r}$ & 10,000 & 387 & 706 & 22.0 & 25.2 & 26.6 & $\mathrm{r}$ \\
\hline $\mathrm{s}$ & 20,000 & 171 & 280 & 5.4 & 63.4 & 54.4 & $\mathrm{~S}$ \\
\hline $\mathrm{t}$ & 0 & 290 & 502 & 32.0 & 34.0 & 39.8 & $\mathrm{t}$ \\
\hline $\mathrm{u}$ & 0 & 331 & 556 & 15.4 & 19.9 & 17.4 & $\mathrm{u}$ \\
\hline V & 28300 & 401 & 859 & 52.2 & 52.8 & 48.9 & $\mathrm{~V}$ \\
\hline$\downarrow$ & $\downarrow$ & $\downarrow$ & $\downarrow$ & $\downarrow$ & $\downarrow$ & $\downarrow$ & $\downarrow$ \\
\hline
\end{tabular}

t-Age $=$ aging time

$0.2 \% \mathrm{YS}=0.2 \%$ yield stress

UTS $=$ ultimate tensile stress

UnifStn = uniform strain, the strain prior to necking

TotalStn $=$ total strain, the strain at rupture

$\mathbf{R A}=$ area of reduction 
Table 1 (cont'd): Existing tensile property data under various testing conditions

\begin{tabular}{|c|c|c|c|}
\hline $\mathbf{a}$ & Source & $\overline{D C}$ & Note \\
\hline $\mathrm{b}$ & McCoy0285T3 & 5 & \\
\hline $\mathrm{c}$ & McCoy0285T3 & 5 & \\
\hline d & McCoy0285T3 & 5 & \\
\hline $\mathrm{e}$ & McCoy0285T3 & 5 & \\
\hline $\mathrm{f}$ & McCoy0285T3 & 5 & \\
\hline $\mathrm{g}$ & McCoy0285T3 & 5 & Broke at gage mark \\
\hline $\mathrm{h}$ & McCoy0285T3 & 5 & \\
\hline $\mathrm{i}$ & McCoy0285T3 & 5 & \\
\hline $\mathrm{j}$ & McCoy0285T3 & 5 & \\
\hline $\mathrm{k}$ & McCoy0285T3 & 5 & \\
\hline 1 & McCoy0285T3 & 5 & \\
\hline $\mathrm{m}$ & McCoy0285T3 & 5 & \\
\hline $\mathrm{n}$ & McCoy0285T3 & 5 & \\
\hline $\mathrm{o}$ & McCoy0285T3 & 5 & Pull rod broke before sample did \\
\hline $\mathrm{p}$ & McCoy0285T3 & 5 & \\
\hline $\mathrm{q}$ & McCoy0285T3 & 5 & \\
\hline $\mathrm{r}$ & McCoy0285T4 & 5 & \\
\hline $\mathrm{S}$ & McCoy0285T4 & 5 & \\
\hline $\mathrm{t}$ & McCoy0285T5 & 5 & \\
\hline $\mathrm{u}$ & McCoy0285T5 & 5 & \\
\hline $\mathrm{V}$ & McCoy0285T7 & 5 & $\begin{array}{l}\text { sample made after } \\
\text { block aged in steam }\end{array}$ \\
\hline$\downarrow$ & $\downarrow$ & $\downarrow$ & $\downarrow$ \\
\hline
\end{tabular}

Source $=$ the tag indicating data source as discussed in Section 2.2

DC $=$ data classification as discussed in Section 2.1

Note $=$ comments or explanations for the data point of the test 
Table 2: Existing weld tensile property data under various testing conditions

\begin{tabular}{|c|c|c|c|c|c|}
\hline BMID & BM Form & BM Treat & WMID & WM Form & $\overline{\mathbf{a}}$ \\
\hline XX14A6UK & $13 \mathrm{~mm}$ plate & $\begin{array}{l}\text { solution } \\
\text { annealed }\end{array}$ & XX09A9UK & $1.1 \mathrm{~mm}$ dia. wire & $\mathrm{b}$ \\
\hline XX14A6UK & $13 \mathrm{~mm}$ plate & $\begin{array}{l}\text { solution } \\
\text { annealed }\end{array}$ & XX09A9UK & $1.1 \mathrm{~mm}$ dia. wire & $\mathrm{c}$ \\
\hline XX14A6UK & $13 \mathrm{~mm}$ plate & $\begin{array}{l}\text { solution } \\
\text { annealed }\end{array}$ & XX09A9UK & $1.1 \mathrm{~mm}$ dia. wire & d \\
\hline XX14A6UK & $13 \mathrm{~mm}$ plate & $\begin{array}{l}\text { solution } \\
\text { annealed }\end{array}$ & XX09A9UK & $1.1 \mathrm{~mm}$ dia. wire & $\mathrm{e}$ \\
\hline XX14A6UK & $13 \mathrm{~mm}$ plate & $\begin{array}{l}\text { solution } \\
\text { annealed }\end{array}$ & XX09A9UK & $1.1 \mathrm{~mm}$ dia. wire & $\mathrm{f}$ \\
\hline XX14A6UK & $13 \mathrm{~mm}$ plate & $\begin{array}{l}\text { solution } \\
\text { annealed }\end{array}$ & XX09A9UK & $1.1 \mathrm{~mm}$ dia. wire & g \\
\hline XX14A6UK & $13 \mathrm{~mm}$ plate & $\begin{array}{l}\text { solution } \\
\text { annealed }\end{array}$ & XX09A9UK & $1.1 \mathrm{~mm}$ dia. wire & $\mathrm{h}$ \\
\hline XX14A6UK & $13 \mathrm{~mm}$ plate & $\begin{array}{l}\text { solution } \\
\text { annealed }\end{array}$ & XX09A9UK & $1.1 \mathrm{~mm}$ dia. wire & $\mathrm{i}$ \\
\hline XX14A6UK & $13 \mathrm{~mm}$ plate & $\begin{array}{l}\text { solution } \\
\text { annealed }\end{array}$ & XX09A9UK & $1.1 \mathrm{~mm}$ dia. wire & $\mathrm{j}$ \\
\hline XX14A6UK & $13 \mathrm{~mm}$ plate & $\begin{array}{c}\text { solution } \\
\text { annealed }\end{array}$ & XX09A9UK & $1.1 \mathrm{~mm}$ dia. wire & $\mathrm{k}$ \\
\hline XX14A6UK & $13 \mathrm{~mm}$ plate & $\begin{array}{c}\text { solution } \\
\text { annealed }\end{array}$ & XX09A9UK & $1.1 \mathrm{~mm}$ dia. wire & 1 \\
\hline XX14A6UK & $13 \mathrm{~mm}$ plate & $\begin{array}{l}\text { solution } \\
\text { annealed }\end{array}$ & XX09A9UK & $1.1 \mathrm{~mm}$ dia. wire & $\mathrm{m}$ \\
\hline XX14A6UK & $13 \mathrm{~mm}$ plate & $\begin{array}{l}\text { solution } \\
\text { annealed }\end{array}$ & XX09A9UK & $1.1 \mathrm{~mm}$ dia. wire & $\mathrm{n}$ \\
\hline XX14A6UK & $13 \mathrm{~mm}$ plate & $\begin{array}{l}\text { solution } \\
\text { annealed }\end{array}$ & XX09A9UK & $1.1 \mathrm{~mm}$ dia. wire & $\mathrm{o}$ \\
\hline$\downarrow$ & $\downarrow$ & $\downarrow$ & $\downarrow$ & $\downarrow$ & $\downarrow$ \\
\hline
\end{tabular}

BMID = heat identification of the base metal before welding

BM Form = product form of the base metal before welding

BM Treat $=$ heat treatment of the base metal

WMID = heat identification of the weld metal

WM Form = product form of the weld metal 
Table 2 (cont'd): Existing weld tensile property data under various testing conditions

\begin{tabular}{|c|c|c|c|c|c|c|c|}
\hline $\mathbf{a}$ & $\mathbf{W M}$ & $\begin{array}{l}\text { Sample } \\
\text { Content }\end{array}$ & $\begin{array}{c}\text { SampleNoAge } \\
\text { Treat }\end{array}$ & $\begin{array}{l}\text { Sample } \\
\text { AgeEnv }\end{array}$ & $\begin{array}{c}\text { T-SampleAge } \\
{ }^{\circ} \mathrm{C}\end{array}$ & $\begin{array}{c}\text { t-SampleAge } \\
\mathrm{h}\end{array}$ & $\bar{a}$ \\
\hline $\mathrm{b}$ & GTA & W & as welded & N/A & N/A & 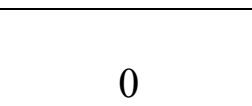 & $\mathrm{b}$ \\
\hline $\mathrm{c}$ & GTA & W & as welded & N/A & N/A & 0 & $\mathrm{c}$ \\
\hline$d$ & GTA & W & as welded & N/A & N/A & 0 & $d$ \\
\hline $\mathrm{e}$ & GTA & W & as welded & N/A & N/A & 0 & $\mathrm{e}$ \\
\hline $\mathrm{f}$ & GTA & W & as welded & $\mathrm{N} / \mathrm{A}$ & N/A & 0 & $\mathrm{f}$ \\
\hline $\mathrm{g}$ & GTA & BWB & N/A & impure $\mathrm{He}$ & 593 & 20,000 & $\mathrm{~g}$ \\
\hline $\mathrm{h}$ & GTA & BWB & $\mathrm{N} / \mathrm{A}$ & impure $\mathrm{He}$ & 704 & 20,000 & $\mathrm{~h}$ \\
\hline $\mathrm{i}$ & GTA & BWB & N/A & impure $\mathrm{He}$ & 871 & 10,000 & $\mathrm{i}$ \\
\hline $\mathrm{j}$ & GTA & BWB & $\mathrm{N} / \mathrm{A}$ & impure $\mathrm{He}$ & 871 & 20,000 & $\mathrm{j}$ \\
\hline $\mathrm{k}$ & GTA & BWB & $\mathrm{N} / \mathrm{A}$ & impure $\mathrm{He}$ & 593 & 10,000 & $\mathrm{k}$ \\
\hline 1 & GTA & BWB & N/A & impure He & 593 & 20,000 & 1 \\
\hline $\mathrm{m}$ & GTA & BWB & N/A & impure $\mathrm{He}$ & 704 & 10,000 & $\mathrm{~m}$ \\
\hline $\mathrm{n}$ & GTA & BWB & N/A & impure He & 704 & 20,000 & $\mathrm{n}$ \\
\hline 0 & GTA & BWB & $\mathrm{N} / \mathrm{A}$ & impure $\mathrm{He}$ & 871 & 10,000 & $\mathrm{O}$ \\
\hline$\downarrow$ & $\downarrow$ & $\downarrow$ & $\downarrow$ & $\downarrow$ & $\downarrow$ & $\downarrow$ & $\downarrow$ \\
\hline
\end{tabular}

$\mathbf{W M}=$ welding method to produce the sample weld: GTA = gas tungsten arc welding Sample Content $=$ composition of the sample: $\mathrm{W}=$ weld metal, $\mathrm{B}=$ base metal, $\mathrm{BWB}=$ weldment, the sample was machined across the weld to include the weld in the middle and base metal on both sides

SampleNoAge Treat $=$ heat treatment of sample that has not been aged

Sample AgeEnv = aging environment of aged sample

T-SampleAge $=$ sample aging temperature

t-SampleAge $=$ sample aging time 
ASSESSMENT OF EXISTING ALLOY 617 DATA FOR GEN IV NUCLEAR REACTOR APPLICATIONS

Table 2 (cont'd): Existing weld tensile property data under various testing conditions

\begin{tabular}{|c|c|c|c|c|c|c|c|c|}
\hline $\mathbf{a}$ & Test ID & $\begin{array}{c}\text { T-Test } \\
{ }^{\circ} \mathrm{C}\end{array}$ & $\begin{array}{c}0.2 \% Y S \\
\text { MPa }\end{array}$ & $\begin{array}{l}\text { UTS } \\
\text { MPa }\end{array}$ & $\begin{array}{c}\text { UnifStn } \\
\%\end{array}$ & $\begin{array}{c}\text { TotalStn } \\
\%\end{array}$ & $\begin{array}{c}\text { RA } \\
\%\end{array}$ & $\mathbf{a}$ \\
\hline $\mathrm{b}$ & - & 25 & 537 & 813 & 32.4 & 35.6 & 54.6 & $\mathrm{~b}$ \\
\hline $\mathrm{c}$ & - & 593 & 393 & 601 & 31.3 & 32.2 & 45.6 & $\mathrm{c}$ \\
\hline $\mathrm{d}$ & - & 649 & 367 & 567 & 35.7 & 36.8 & 38.4 & $\mathrm{~d}$ \\
\hline $\mathrm{e}$ & - & 760 & 412 & 547 & 10.3 & 25.7 & 28.8 & $\mathrm{e}$ \\
\hline $\mathrm{f}$ & - & 871 & 272 & 288 & 6.0 & 42.8 & 71.1 & $\mathrm{f}$ \\
\hline $\mathrm{g}$ & $\mathrm{I}-161$ & 24 & 676 & 1020 & 19.5 & 19.9 & 10.6 & $\mathrm{~g}$ \\
\hline $\mathrm{h}$ & $\mathrm{I}-165$ & 24 & 626 & 867 & 5.0 & 5.2 & 8.3 & $\mathrm{~h}$ \\
\hline $\mathrm{i}$ & I-156 & 24 & 394 & 450 & 0.7 & 0.7 & 3.2 & $\mathrm{i}$ \\
\hline $\mathrm{j}$ & $\mathrm{I}-169$ & 24 & 356 & 476 & 0.8 & 0.8 & 3.3 & $\mathrm{j}$ \\
\hline $\mathrm{k}$ & $\mathrm{I}-168$ & 593 & 259 & 514 & 29.0 & 29.4 & 33.3 & $\mathrm{k}$ \\
\hline 1 & $\mathrm{I}-160$ & 593 & 623 & 800 & 15.2 & 16.2 & 26.6 & 1 \\
\hline $\mathrm{m}$ & $\mathrm{I}-153$ & 704 & 460 & 756 & 15.7 & 19.9 & 35.0 & $\mathrm{~m}$ \\
\hline $\mathrm{n}$ & $\mathrm{I}-164$ & 704 & 445 & 740 & 12.2 & 15.6 & 8.5 & $\mathrm{n}$ \\
\hline $\mathrm{O}$ & $\mathrm{I}-157$ & 871 & 223 & 270 & 6.0 & 26.8 & 56.0 & o \\
\hline$\downarrow$ & $\downarrow$ & $\downarrow$ & $\downarrow$ & $\downarrow$ & $\downarrow$ & $\downarrow$ & $\downarrow$ & $\downarrow$ \\
\hline
\end{tabular}

Test ID $=$ identification of the test that generated the data

T-Test $=$ test temperature

$\mathbf{0 . 2} \% \mathrm{YS}=0.2 \%$ yield stress

UTS $=$ ultimate tensile stress

UnifStn = uniform strain, the strain prior to necking

TotalStn $=$ total strain, the strain at rupture

$\mathbf{R A}=$ area of reduction 
Table 2 (cont'd). Existing weld tensile property data under various testing conditions

\begin{tabular}{|c|c|c|c|c|}
\hline $\bar{a}$ & Source & $\overline{\text { FL }}$ & $\overline{D C}$ & Note \\
\hline $\mathrm{b}$ & McCoy0285T8 & N/A & 5 & \\
\hline $\mathrm{c}$ & МcCoy0285T8 & N/A & 5 & \\
\hline $\mathrm{d}$ & McCoy0285T8 & N/A & 5 & \\
\hline $\mathrm{e}$ & МcCoy0285T8 & N/A & 5 & \\
\hline $\mathrm{f}$ & МcCoy0285T8 & $\mathrm{N} / \mathrm{A}$ & 5 & \\
\hline g & МcCoy0285T9 & B & 5 & \\
\hline $\mathrm{h}$ & МcСoy0285T9 & B & 5 & \\
\hline $\mathrm{i}$ & МcСoy0285T9 & B & 5 & \\
\hline $\mathrm{j}$ & МcСoy0285T9 & B & 5 & \\
\hline $\mathrm{k}$ & МcСoy0285T9 & W & 5 & \\
\hline 1 & МcСoy0285T9 & B & 5 & \\
\hline $\mathrm{m}$ & МcCoy0285T9 & W & 5 & \\
\hline $\mathrm{n}$ & МcСoy0285T9 & B & 5 & \\
\hline o & МcCoy0285T9 & $\mathrm{W}$ & 5 & \\
\hline$\downarrow$ & $\downarrow$ & $\downarrow$ & $\downarrow$ & $\downarrow$ \\
\hline
\end{tabular}

Source $=$ the tag indicating data source as discussed in Section 2.2

$\mathbf{F L}=$ failure location in the sample: $\mathrm{W}=$ weld, $\mathrm{B}=$ base metal

DC $=$ data classification as discussed in Section 2.1

Note $=$ comments or explanations for the data of the test 
Table 3: Existing creep property data under various testing conditions

\begin{tabular}{|c|c|c|c|c|c|c|c|c|}
\hline Heat & Form & $\begin{array}{c}\text { Test } \\
\text { ID }\end{array}$ & $\begin{array}{c}\text { T-Test } \\
{ }^{\circ} \mathrm{C}\end{array}$ & $\begin{array}{c}\text { TestSts } \\
\text { MPa }\end{array}$ & $\begin{array}{l}\text { Test } \\
\text { Env }\end{array}$ & $\begin{array}{c}\text { UnAged } \\
\text { Treat }\end{array}$ & AgeEnv & $\mathbf{a}$ \\
\hline XX01A3US & $13 \mathrm{~mm}$ plate & 22188 & 593 & 414 & Air & $\begin{array}{c}\text { solution } \\
\text { annealed }\end{array}$ & N/A & $b$ \\
\hline XX01A3US & $13 \mathrm{~mm}$ plate & 20531 & 593 & 414 & $\mathrm{He}$ & $\begin{array}{l}\text { solution } \\
\text { annealed }\end{array}$ & N/A & $\mathrm{c}$ \\
\hline XX01A3US & $13 \mathrm{~mm}$ plate & 20521 & 593 & 414 & $\mathrm{He}$ & N/A & $\begin{array}{c}\text { impure } \\
\mathrm{He}\end{array}$ & $\mathrm{d}$ \\
\hline XX01A3US & $13 \mathrm{~mm}$ plate & 22645 & 593 & 414 & $\mathrm{He}$ & N/A & $\begin{array}{c}\text { impure } \\
\mathrm{He}\end{array}$ & $\mathrm{e}$ \\
\hline XX01A3US & $13 \mathrm{~mm}$ plate & 22200 & 593 & 345 & $\mathrm{He}$ & $\mathrm{N} / \mathrm{A}$ & $\begin{array}{c}\text { impure } \\
\mathrm{He}\end{array}$ & $\mathrm{f}$ \\
\hline XX09A4UK & $9.5 \mathrm{~mm}$ plate & 23088 & 593 & 345 & Air & $\begin{array}{c}\text { solution } \\
\text { annealed }\end{array}$ & $\mathrm{N} / \mathrm{A}$ & $\mathrm{g}$ \\
\hline XX01A3US & $13 \mathrm{~mm}$ plate & 19106 & 649 & 414 & $\mathrm{He}$ & $\begin{array}{c}\text { solution } \\
\text { annealed }\end{array}$ & $\mathrm{N} / \mathrm{A}$ & $\mathrm{h}$ \\
\hline XX01A3US & $13 \mathrm{~mm}$ plate & 21589 & 649 & 345 & Air & $\mathrm{N} / \mathrm{A}$ & inert & $\mathrm{i}$ \\
\hline XX01A3US & $13 \mathrm{~mm}$ plate & 21610 & 649 & 345 & Air & $\mathrm{N} / \mathrm{A}$ & inert & $\mathrm{j}$ \\
\hline XX01A3US & $13 \mathrm{~mm}$ plate & 19182 & 649 & 276 & $\mathrm{He}$ & $\begin{array}{c}\text { solution } \\
\text { annealed }\end{array}$ & $\mathrm{N} / \mathrm{A}$ & $\mathrm{k}$ \\
\hline XX01A3US & $13 \mathrm{~mm}$ plate & 18483 & 649 & 276 & $\mathrm{He}$ & $\begin{array}{c}\text { solution } \\
\text { annealed }\end{array}$ & $\mathrm{N} / \mathrm{A}$ & 1 \\
\hline XX01A3US & $13 \mathrm{~mm}$ plate & 18440 & 649 & 276 & Air & $\begin{array}{c}\text { solution } \\
\text { annealed }\end{array}$ & $\mathrm{N} / \mathrm{A}$ & $\mathrm{m}$ \\
\hline XX01A3US & $13 \mathrm{~mm}$ plate & 19182 & 649 & 207 & $\mathrm{He}$ & $\begin{array}{c}\text { solution } \\
\text { annealed }\end{array}$ & N/A & $\mathrm{n}$ \\
\hline XX09A4UK & $9.5 \mathrm{~mm}$ plate & 23085 & 649 & 276 & Air & $\begin{array}{l}\text { solution } \\
\text { annealed }\end{array}$ & N/A & $\mathrm{o}$ \\
\hline$\downarrow$ & $\downarrow$ & $\downarrow$ & $\downarrow$ & $\downarrow$ & $\downarrow$ & $\downarrow$ & $\downarrow$ & $\downarrow$ \\
\hline
\end{tabular}

Heat $=$ heat identification

Form $=$ product form

Test ID $=$ identification of the test that generated the data

T-Test $=$ test temperature

TestSts $=$ test stress

TestEnv $=$ test environment

UnAged Treat $=$ heat treatment for sample without aging

AgeEnv = aging environment for aged sample 
ASSESSMENT OF EXISTING ALLOY 617 DATA FOR GEN IV NUCLEAR REACTOR APPLICATIONS

Table 3 (cont'd): Existing creep property data under various testing conditions

\begin{tabular}{|c|c|c|c|c|c|c|c|c|c|}
\hline $\mathbf{a}$ & $\begin{array}{c}\text { T-Age } \\
{ }^{\circ} \mathrm{C}\end{array}$ & $\begin{array}{c}\text { t-Age } \\
\text { h }\end{array}$ & $\begin{array}{c}\text { t-1\%Stn } \\
\text { h }\end{array}$ & $\begin{array}{c}\text { t-2\%Stn } \\
h\end{array}$ & $\begin{array}{c}\text { t-5\%Stn } \\
\text { h }\end{array}$ & $\begin{array}{c}\text { t-TerStn } \\
\text { h }\end{array}$ & $\begin{array}{c}\text { t-Rpt } \\
\text { H }\end{array}$ & $\begin{array}{c}\text { MCR } \\
\text { 1/h }\end{array}$ & $\mathbf{a}$ \\
\hline $\mathrm{b}$ & N/A & N/A & 20 & - & 3,350 & 3,445 & 3,570 & $4.8 \mathrm{E}-6$ & $\mathrm{~b}$ \\
\hline $\mathrm{c}$ & N/A & N/A & 380 & 1,700 & - & 4,534 & 4,534 & 4.9 E-6 & $\mathrm{c}$ \\
\hline $\mathrm{d}$ & 593 & 10,000 & 500 & - & - & - & 548 & $1.0 \mathrm{E}-5$ & $\mathrm{~d}$ \\
\hline $\mathrm{e}$ & 593 & 20,000 & 2,000 & - & - & 2,600 & 2,676 & 2.4 E-6 & $\mathrm{e}$ \\
\hline $\mathrm{f}$ & 593 & 10,000 & - & - & - & - & 5,629 & $5.5 \mathrm{E}-7$ & $\mathrm{f}$ \\
\hline $\mathrm{g}$ & N/A & $\mathrm{N} / \mathrm{A}$ & - & - & - & - & 2,107 & $1.4 \mathrm{E}-6$ & $\mathrm{~g}$ \\
\hline $\mathrm{h}$ & N/A & $\mathrm{N} / \mathrm{A}$ & 0.5 & 5 & 145 & 168 & 186 & $1.6 \mathrm{E}-4$ & $\mathrm{~h}$ \\
\hline $\mathrm{i}$ & 482 & 28,300 & 280 & 1,100 & - & 1,700 & 1,813 & $1.3 \mathrm{E}-5$ & $\mathrm{i}$ \\
\hline $\mathrm{j}$ & 538 & 28,300 & 189 & 837 & - & - & 1,063 & 2.4 E-5 & $\mathrm{j}$ \\
\hline $\mathrm{k}$ & N/A & N/A & 5,000 & 8,600 & 15,100 & 8,000 & 16,718 & 2.0 E-6 & $\mathrm{k}$ \\
\hline 1 & N/A & $\mathrm{N} / \mathrm{A}$ & 7,600 & - & - & - & 15,850 & $1.1 \mathrm{E}-6$ & 1 \\
\hline $\mathrm{m}$ & N/A & N/A & 8,850 & 16,480 & 25,510 & 16,000 & 25,566 & $9.3 \mathrm{E}-7$ & $\mathrm{~m}$ \\
\hline $\mathrm{n}$ & N/A & $\mathrm{N} / \mathrm{A}$ & - & - & - & - & 26,515 & 9.9 E-8 & $\mathrm{n}$ \\
\hline 0 & N/A & $\mathrm{N} / \mathrm{A}$ & - & - & - & - & 1,121 & 1.9 E-6 & $\mathrm{o}$ \\
\hline$\downarrow$ & $\downarrow$ & $\downarrow$ & $\downarrow$ & $\downarrow$ & $\downarrow$ & $\downarrow$ & $\downarrow$ & $\downarrow$ & $\downarrow$ \\
\hline
\end{tabular}

T-Age $=$ aging temperature

t-Age $=$ aging time

t-1\%Stn $=$ time for reaching $1 \%$ creep strain

$\mathbf{t}-2 \%$ Stn $=$ time for reaching $2 \%$ creep strain

$\mathbf{t}-\mathbf{5 \%} \mathbf{S t n}=$ time for reaching $5 \%$ creep strain

t-TerStn $=$ time for reaching tertiary creep strain

$\mathbf{t}-\mathbf{R p t}=$ time for creep rupture

$\mathbf{M C R}=$ minimum creep rate 
Table 3 (cont'd): Existing creep property data under various testing conditions

\begin{tabular}{c|c|c|c|l|l|l|}
\hline a & $\begin{array}{c}\text { LdgStn } \\
\text { \% }\end{array}$ & $\begin{array}{c}\text { CpStn } \\
\text { \% }\end{array}$ & $\begin{array}{c}\text { RA } \\
\text { \% }\end{array}$ & Source & DC & Note \\
\hline $\mathrm{b}$ & 16 & 6.9 & 18.2 & McCoy0285T14 & 5 & \\
\hline $\mathrm{c}$ & 18 & 3.7 & 21.3 & McCoy0285T14 & 5 & \\
\hline $\mathrm{d}$ & & 2.2 & 22.0 & McCoy0285T14 & 5 & \\
\hline $\mathrm{e}$ & 1.3 & 3.9 & 12.0 & McCoy0285T14 & 5 & \\
\hline $\mathrm{f}$ & 4.4 & 5.5 & 14.4 & McCoy0285T14 & 5 & \\
\hline $\mathrm{g}$ & & & & & & \\
\hline $\mathrm{h}$ & 9.3 & 3.4 & 15.2 & McCoy0285T14 & 5 & \\
\hline $\mathrm{i}$ & 8.4 & 5.6 & 13.5 & McCoy0285T14 & 5 & \\
\hline $\mathrm{j}$ & 6.8 & 6.5 & 13.5 & McCoy0285T14 & 5 & \\
\hline $\mathrm{k}$ & & & & & & \\
\hline $\mathrm{n}$ & 0.03 & 9.3 & 14.9 & McCoy0285T14 & 5 & \\
\hline $\mathrm{l}$ & & & & & & \\
\hline $\mathrm{m}$ & 2.5 & 6.1 & 8.3 & McCoy0285T14 & 5 & \\
\hline & 1.5 & 6.4 & 15.7 & McCoy0285T14 & 5 & \\
\hline & 0.3 & 0.3 & - & McCoy0285T14 & 5 & test reloaded at a higher stress \\
\hline & 1.5 & 1.4 & 4.5 & McCoy0285T14 & 5 & \\
\hline & $\downarrow$ & $\downarrow$ & & $\downarrow$ & \\
\hline
\end{tabular}

LdgStn $=$ loading strain

CpStn $=$ creep rupture strain

$\mathbf{R A}=$ area of reduction

Source $=$ the tag indicating data source as discussed in Section 2.2

DC = data classification as discussed in Section 2.1

Note $=$ comments or explanations for the data point of the test 
Table 4: Existing weld creep property data under various testing conditions

\begin{tabular}{|c|c|c|c|c|c|c|}
\hline BMID & BM Form & BM Treat & WMID & WM Form & WM & $\mathbf{a}$ \\
\hline XX14A6UK & $13 \mathrm{~mm}$ plate & $\begin{array}{c}\text { solution } \\
\text { annealed }\end{array}$ & XX09A9UK & $1.1 \mathrm{~mm}$ dia. wire & GTA & b \\
\hline XX14A6UK & $13 \mathrm{~mm}$ plate & $\begin{array}{l}\text { solution } \\
\text { annealed }\end{array}$ & XX09A9UK & $1.1 \mathrm{~mm}$ dia. wire & GTA & c \\
\hline XX14A6UK & $13 \mathrm{~mm}$ plate & $\begin{array}{c}\text { solution } \\
\text { annealed }\end{array}$ & XX09A9UK & $1.1 \mathrm{~mm}$ dia. wire & GTA & $\mathrm{d}$ \\
\hline XX14A6UK & $13 \mathrm{~mm}$ plate & $\begin{array}{l}\text { solution } \\
\text { annealed }\end{array}$ & XX09A9UK & $1.1 \mathrm{~mm}$ dia. wire & GTA & e \\
\hline XX14A6UK & $13 \mathrm{~mm}$ plate & $\begin{array}{c}\text { solution } \\
\text { annealed }\end{array}$ & XX09A9UK & $1.1 \mathrm{~mm}$ dia. wire & GTA & $\mathrm{f}$ \\
\hline XX14A6UK & $13 \mathrm{~mm}$ plate & $\begin{array}{c}\text { solution } \\
\text { annealed }\end{array}$ & XX09A9UK & $1.1 \mathrm{~mm}$ dia. wire & GTA & $\mathrm{g}$ \\
\hline XX14A6UK & $13 \mathrm{~mm}$ plate & $\begin{array}{c}\text { solution } \\
\text { annealed }\end{array}$ & XX09A9UK & $1.1 \mathrm{~mm}$ dia. wire & GTA & $\mathrm{h}$ \\
\hline XX14A6UK & $13 \mathrm{~mm}$ plate & $\begin{array}{c}\text { solution } \\
\text { annealed }\end{array}$ & XX09A9UK & $1.1 \mathrm{~mm}$ dia. wire & GTA & $\mathrm{i}$ \\
\hline XX14A6UK & $13 \mathrm{~mm}$ plate & $\begin{array}{c}\text { solution } \\
\text { annealed }\end{array}$ & XX09A9UK & $1.1 \mathrm{~mm}$ dia. wire & GTA & $\mathrm{j}$ \\
\hline XX14A6UK & $13 \mathrm{~mm}$ plate & $\begin{array}{c}\text { solution } \\
\text { annealed }\end{array}$ & XX09A9UK & $1.1 \mathrm{~mm}$ dia. wire & GTA & $\mathrm{k}$ \\
\hline XX14A6UK & $13 \mathrm{~mm}$ plate & $\begin{array}{c}\text { solution } \\
\text { annealed }\end{array}$ & XX09A9UK & $1.1 \mathrm{~mm}$ dia. wire & GTA & 1 \\
\hline XX14A6UK & $13 \mathrm{~mm}$ plate & $\begin{array}{c}\text { solution } \\
\text { annealed }\end{array}$ & XX09A9UK & $1.1 \mathrm{~mm}$ dia. wire & GTA & $\mathrm{m}$ \\
\hline XX14A6UK & $13 \mathrm{~mm}$ plate & $\begin{array}{l}\text { solution } \\
\text { annealed }\end{array}$ & XX09A9UK & $1.1 \mathrm{~mm}$ dia. wire & GTA & $\mathrm{n}$ \\
\hline$\downarrow$ & $\downarrow$ & $\downarrow$ & $\downarrow$ & $\downarrow$ & $\downarrow$ & $\downarrow$ \\
\hline
\end{tabular}

BMID $=$ heat identification of the base metal before welding

BM Form = product form of the base metal before welding

BM Treat $=$ heat treatment of the base metal

WMID = heat identification of the weld metal

WM Form = product form of the weld metal

$\mathbf{W M}=$ welding method to produce the sample weld: GTA = gas tungsten arc welding 
Table 4 (cont'd): Existing weld creep property data under various testing conditions

\begin{tabular}{|c|c|c|c|c|c|c|c|}
\hline $\mathbf{a}$ & $\begin{array}{l}\text { Sample } \\
\text { Content }\end{array}$ & $\begin{array}{c}\text { SampleNoAge } \\
\text { Treat }\end{array}$ & $\begin{array}{l}\text { Sample } \\
\text { AgeEnv }\end{array}$ & $\begin{array}{l}\text { T-SampleAge } \\
{ }^{\circ} \mathrm{C}\end{array}$ & $\begin{array}{c}\text { t-SampleAge } \\
\text { h }\end{array}$ & $\begin{array}{c}\text { Test } \\
\text { ID }\end{array}$ & $\mathbf{a}$ \\
\hline $\mathrm{b}$ & W & as welded & $\mathrm{N} / \mathrm{A}$ & $\mathrm{N} / \mathrm{A}$ & 0 & 20515 & $\mathrm{~b}$ \\
\hline $\mathrm{c}$ & W & as welded & $\mathrm{N} / \mathrm{A}$ & $\mathrm{N} / \mathrm{A}$ & 0 & none & $\mathrm{c}$ \\
\hline $\mathrm{d}$ & W & as welded & N/A & N/A & 0 & 23071 & $\mathrm{~d}$ \\
\hline $\mathrm{e}$ & W & as welded & $\mathrm{N} / \mathrm{A}$ & N/A & 0 & 21399 & $\mathrm{e}$ \\
\hline $\mathrm{f}$ & W & as welded & N/A & N/A & 0 & 20513 & $\mathrm{f}$ \\
\hline $\mathrm{g}$ & W & as welded & $\mathrm{N} / \mathrm{A}$ & $\mathrm{N} / \mathrm{A}$ & 0 & 20553 & $\mathrm{~g}$ \\
\hline $\mathrm{h}$ & W & as welded & $\mathrm{N} / \mathrm{A}$ & $\mathrm{N} / \mathrm{A}$ & 0 & 22741 & $\mathrm{~h}$ \\
\hline $\mathrm{i}$ & W & as welded & $\mathrm{N} / \mathrm{A}$ & $\mathrm{N} / \mathrm{A}$ & 0 & 22763 & $\mathrm{i}$ \\
\hline $\mathrm{j}$ & W & as welded & $\mathrm{N} / \mathrm{A}$ & $\mathrm{N} / \mathrm{A}$ & 0 & 20735 & $\mathrm{j}$ \\
\hline $\mathrm{k}$ & W & as welded & $\mathrm{N} / \mathrm{A}$ & $\mathrm{N} / \mathrm{A}$ & 0 & 20533 & $\mathrm{k}$ \\
\hline 1 & BWB & $\mathrm{N} / \mathrm{A}$ & Impure $\mathrm{He}$ & 593 & 10,000 & 20522 & 1 \\
\hline $\mathrm{m}$ & BWB & N/A & Impure He & 704 & 10,000 & 20528 & $\mathrm{~m}$ \\
\hline $\mathrm{n}$ & BWB & N/A & Impure $\mathrm{He}$ & 704 & 20,000 & 21612 & $\mathrm{n}$ \\
\hline$\downarrow$ & $\downarrow$ & $\downarrow$ & $\downarrow$ & $\downarrow$ & $\downarrow$ & $\downarrow$ & $\downarrow$ \\
\hline
\end{tabular}

Sample Content $=$ composition of the sample: $\mathrm{W}=$ weld metal, $\mathrm{B}=$ base metal, $\mathrm{BWB}=$ weldment, the sample was machined across the weld to include the weld in the middle and base metal on both sides

SampleNoAge Treat $=$ heat treatment of sample that has not been aged

Sample AgeEnv = aging environment of aged sample

T-SampleAge $=$ sample aging temperature

t-SampleAge $=$ sample aging time

Test ID $=$ identification of the test that generated the data 
ASSESSMENT OF EXISTING ALLOY 617 DATA FOR GEN IV NUCLEAR REACTOR APPLICATIONS

Table 4 (cont'd): Existing weld creep property data under various testing conditions

\begin{tabular}{|c|c|c|c|c|c|c|c|c|c|}
\hline $\mathbf{a}$ & $\begin{array}{c}\text { T-Test } \\
{ }^{\circ} \mathrm{C}\end{array}$ & $\begin{array}{c}\text { Sts-Test } \\
\text { MPa }\end{array}$ & $\begin{array}{l}\text { Test } \\
\text { Env }\end{array}$ & $\begin{array}{c}\text { t-1\%Stn } \\
\text { H }\end{array}$ & $\begin{array}{c}\text { t-2\%Stn } \\
\text { h }\end{array}$ & $\begin{array}{c}\text { t-5\%Stn } \\
h\end{array}$ & $\begin{array}{c}\text { t-TerStn } \\
\text { h }\end{array}$ & $\begin{array}{c}\text { t-Rpt } \\
\text { h }\end{array}$ & $\mathbf{a}$ \\
\hline $\mathrm{b}$ & 593 & 345 & $\mathrm{He}$ & - & - & - & - & $>13,726$ & $\mathrm{~b}$ \\
\hline $\mathrm{c}$ & 593 & 414 & $\mathrm{He}$ & - & - & - & - & 1,296 & $\mathrm{c}$ \\
\hline $\mathrm{d}$ & 593 & 414 & $\mathrm{He}$ & - & - & - & - & 2,034 & $\mathrm{~d}$ \\
\hline $\mathrm{e}$ & 649 & 345 & $\mathrm{He}$ & 525 & - & - & - & 526 & $\mathrm{e}$ \\
\hline $\mathrm{f}$ & 649 & 276 & $\mathrm{He}$ & - & - & - & - & 3,467 & $\mathrm{f}$ \\
\hline $\mathrm{g}$ & 649 & 242 & $\mathrm{He}$ & 15,400 & - & - & - & 18,182 & $\mathrm{~g}$ \\
\hline $\mathrm{h}$ & 704 & 276 & $\mathrm{He}$ & 775 & - & - & 785 & 790 & $\mathrm{~h}$ \\
\hline $\mathrm{i}$ & 704 & 207 & $\mathrm{He}$ & 4,250 & 10,500 & 14,900 & 6,000 & 14,978 & $\mathrm{i}$ \\
\hline $\mathrm{j}$ & 760 & 138 & $\mathrm{He}$ & 2,900 & 3,750 & 4,750 & 2,600 & 5,190 & $\mathrm{j}$ \\
\hline $\mathrm{k}$ & 871 & 69 & $\mathrm{He}$ & 1,467 & 1,570 & - & - & 1,623 & $\mathrm{k}$ \\
\hline 1 & 593 & 414 & $\mathrm{He}$ & - & - & - & - & 645 & 1 \\
\hline $\mathrm{m}$ & 704 & 207 & $\mathrm{He}$ & 4,830 & 6,380 & - & 5,100 & 6,932 & $\mathrm{~m}$ \\
\hline $\mathrm{n}$ & 704 & 207 & $\mathrm{He}$ & 1,100 & 3,250 & 5,470 & 3,025 & 5,496 & $\mathrm{n}$ \\
\hline$\downarrow$ & $\downarrow$ & $\downarrow$ & $\downarrow$ & $\downarrow$ & $\downarrow$ & $\downarrow$ & $\downarrow$ & $\downarrow$ & $\downarrow$ \\
\hline
\end{tabular}

T-Test $=$ test temperature

Sts-Test $=$ test stress

$\mathbf{t}-\mathbf{1 \%}$ Stn $=$ time for reaching $1 \%$ creep strain

$\mathbf{t}-2 \%$ Stn $=$ time for reaching $2 \%$ creep strain

$\mathbf{t}-\mathbf{5 \%}$ Stn $=$ time for reaching $5 \%$ creep strain

$\mathbf{t}$-TerStn $=$ time for reaching tertiary creep strain

$\mathbf{t}-\mathbf{R p t}=$ creep rupture time 
ASSESSMENT OF EXISTING ALLOY 617 DATA FOR GEN IV NUCLEAR REACTOR APPLICATIONS

Table 4 (cont'd): Existing weld creep property data under various testing conditions

\begin{tabular}{|c|c|c|c|c|c|c|c|c|}
\hline $\mathbf{a}$ & $\begin{array}{c}\text { MCR } \\
\text { 1/h }\end{array}$ & $\begin{array}{c}\text { LdgStn } \\
\% \\
\end{array}$ & $\begin{array}{c}\text { CpStn } \\
\% \\
\end{array}$ & $\begin{array}{c}\text { RA } \\
\%\end{array}$ & Source & FL & DC & Note \\
\hline $\mathrm{b}$ & $1.2 \mathrm{E}-7$ & 0.6 & 0.4 & - & McСoy0285T15 & N/A & 5 & $\begin{array}{l}\text { Discontinued } \\
\text { before failure }\end{array}$ \\
\hline $\mathrm{c}$ & - & 0.09 & 0.1 & - & McCoy0285T15 & N/A & 5 & \\
\hline d & $1.3 \mathrm{E}-6$ & 0.72 & 2 & 5.1 & МcСoy0285T15 & N/A & 5 & \\
\hline $\mathrm{e}$ & $8.1 \mathrm{E}-6$ & 0.1 & 2.6 & 3.3 & МcСoy0285T15 & N/A & 5 & \\
\hline $\mathrm{f}$ & $5.4 \mathrm{E}-7$ & 0.1 & 2.3 & 1.4 & МcСoy0285T15 & N/A & 5 & \\
\hline $\mathrm{g}$ & $4.7 \mathrm{E}-7$ & 0.2 & 1.8 & 2.9 & МcСoy0285T15 & N/A & 5 & \\
\hline $\mathrm{h}$ & $8.0 \mathrm{E}-6$ & 0.5 & 4.4 & 5.9 & McСoy0285T15 & N/A & 5 & \\
\hline $\mathrm{i}$ & $5.5 \mathrm{E}-7$ & 0.04 & 7.7 & 14.1 & МcСoy0285T15 & N/A & 5 & \\
\hline $\mathrm{j}$ & $1.8 \mathrm{E}-6$ & - & 17.1 & 43.5 & МcСoy0285T15 & N/A & 5 & \\
\hline $\mathrm{k}$ & - & 0.07 & 10.7 & 21 & МcСoy0285T15 & N/A & 5 & \\
\hline 1 & - & - & 1.7 & 5.2 & McCoy0285T16 & $\mathrm{W}$ & 5 & \\
\hline $\mathrm{m}$ & 2.6 E-6 & 0.3 & 3.7 & 17.1 & МcСоу0285T16 & W & 5 & \\
\hline $\mathrm{n}$ & 3.7 E-6 & 0 & 6.5 & 17.6 & МcСoy0285T16 & W & 5 & \\
\hline$\downarrow$ & $\downarrow$ & $\downarrow$ & $\downarrow$ & $\downarrow$ & $\downarrow$ & $\downarrow$ & $\downarrow$ & $\downarrow$ \\
\hline
\end{tabular}

$\mathbf{M C R}=$ minimum creep rate

LdgStn $=$ loading strain

CpStn $=$ creep rupture strain

$\mathbf{R A}=$ area of reduction

Source $=$ the tag indicating data source as discussed in Section 2.2

$\mathbf{F L}=$ failure location in the sample: $\mathrm{W}=$ weld, $\mathrm{B}=$ base metal

DC $=$ data classification as discussed in Section 2.1

Note $=$ comments or explanations for the data of the test 
Table 5: Existing fatigue property data under various testing conditions

\begin{tabular}{|c|c|c|c|c|c|c|c|c|}
\hline Heat & Form & $\begin{array}{c}\text { Test } \\
\text { ID }\end{array}$ & $\begin{array}{c}\text { NoneAge } \\
\text { Treat } \\
\end{array}$ & AgeEnv & $\begin{array}{c}\text { T-Age } \\
{ }^{\circ} \mathrm{C}\end{array}$ & $\begin{array}{c}\text { t-Age } \\
\text { h }\end{array}$ & $\begin{array}{l}\text { Test } \\
\text { Env }\end{array}$ & $\mathbf{a}$ \\
\hline XX14A6UK & $16 \mathrm{~mm}$ plate & & $\begin{array}{l}\text { Solution } \\
\text { Annealed }\end{array}$ & N/A & N/A & N/A & Air & $\mathrm{b}$ \\
\hline XX14A6UK & $16 \mathrm{~mm}$ plate & & $\begin{array}{c}\text { Solution } \\
\text { Annealed }\end{array}$ & N/A & $\mathrm{N} / \mathrm{A}$ & $\mathrm{N} / \mathrm{A}$ & $\mathrm{He}$ & $\mathrm{c}$ \\
\hline XX14A6UK & $16 \mathrm{~mm}$ plate & & $\begin{array}{c}\text { Solution } \\
\text { Annealed }\end{array}$ & N/A & N/A & $\mathrm{N} / \mathrm{A}$ & $\mathrm{He}$ & $\mathrm{d}$ \\
\hline XX14A6UK & $16 \mathrm{~mm}$ plate & & N/A & $\mathrm{He}$ & 850 & 6000 & $\mathrm{He}$ & $\mathrm{e}$ \\
\hline XX14A6UK & $16 \mathrm{~mm}$ plate & & $\mathrm{N} / \mathrm{A}$ & $\mathrm{He}$ & 950 & 6000 & $\mathrm{He}$ & $\mathrm{f}$ \\
\hline XX14A6UK & $16 \mathrm{~mm}$ plate & & $\begin{array}{c}\text { Solution } \\
\text { Annealed }\end{array}$ & N/A & $\mathrm{N} / \mathrm{A}$ & $\mathrm{N} / \mathrm{A}$ & Air & $\mathrm{g}$ \\
\hline XX14A6UK & $16 \mathrm{~mm}$ plate & & $\begin{array}{c}\text { Solution } \\
\text { Annealed }\end{array}$ & N/A & $\mathrm{N} / \mathrm{A}$ & $\mathrm{N} / \mathrm{A}$ & $\mathrm{He}$ & $\mathrm{h}$ \\
\hline XX14A6UK & $16 \mathrm{~mm}$ plate & & $\begin{array}{c}\text { Solution } \\
\text { Annealed }\end{array}$ & N/A & N/A & $\mathrm{N} / \mathrm{A}$ & $\mathrm{He}$ & $\mathrm{i}$ \\
\hline XX14A6UK & $16 \mathrm{~mm}$ plate & & N/A & $\mathrm{He}$ & 850 & 6000 & $\mathrm{He}$ & $\mathrm{j}$ \\
\hline XX14A6UK & $16 \mathrm{~mm}$ plate & & $\mathrm{N} / \mathrm{A}$ & $\mathrm{He}$ & 950 & 6000 & $\mathrm{He}$ & $\mathrm{k}$ \\
\hline XX63A8UK & $44.5 \mathrm{~mm} \mathrm{rd} \mathrm{bar}$ & & $\begin{array}{c}\text { Solution } \\
\text { Annealed }\end{array}$ & N/A & $\mathrm{N} / \mathrm{A}$ & $\mathrm{N} / \mathrm{A}$ & Air & 1 \\
\hline XX63A8UK & $44.5 \mathrm{~mm}$ rd bar & & $\begin{array}{c}\text { Solution } \\
\text { Annealed }\end{array}$ & N/A & $\mathrm{N} / \mathrm{A}$ & $\mathrm{N} / \mathrm{A}$ & Air & $\mathrm{m}$ \\
\hline XX63A8UK & $44.5 \mathrm{~mm} \mathrm{rd} \mathrm{bar}$ & & $\mathrm{N} / \mathrm{A}$ & $\mathrm{He}$ & 950 & 6000 & $\mathrm{He}$ & $\mathrm{n}$ \\
\hline XX63A8UK & $44.5 \mathrm{~mm} \mathrm{rd} \mathrm{bar}$ & & N/A & $\mathrm{He}$ & 950 & 6000 & $\mathrm{He}$ & 0 \\
\hline XX63A8UK & $44.5 \mathrm{~mm} \mathrm{rd} \mathrm{bar}$ & & $\begin{array}{c}\text { Solution } \\
\text { Annealed }\end{array}$ & N/A & $\mathrm{N} / \mathrm{A}$ & $\mathrm{N} / \mathrm{A}$ & Air & $\mathrm{p}$ \\
\hline XX63A8UK & $44.5 \mathrm{~mm} \mathrm{rd} \mathrm{bar}$ & & $\begin{array}{c}\text { Solution } \\
\text { Annealed }\end{array}$ & N/A & N/A & $\mathrm{N} / \mathrm{A}$ & Air & $q$ \\
\hline XX63A8UK & $44.5 \mathrm{~mm}$ rd bar & & $\begin{array}{l}\text { Solution } \\
\text { Annealed }\end{array}$ & $\mathrm{N} / \mathrm{A}$ & $\mathrm{N} / \mathrm{A}$ & $\mathrm{N} / \mathrm{A}$ & Air & $\mathrm{r}$ \\
\hline XX63A8UK & $44.5 \mathrm{~mm} \mathrm{rd} \mathrm{bar}$ & & $\begin{array}{l}\text { Solution } \\
\text { Annealed }\end{array}$ & $\mathrm{N} / \mathrm{A}$ & $\mathrm{N} / \mathrm{A}$ & $\mathrm{N} / \mathrm{A}$ & Air & $\mathrm{s}$ \\
\hline$\downarrow$ & $\downarrow$ & $\downarrow$ & $\downarrow$ & $\downarrow$ & $\downarrow$ & $\downarrow$ & $\downarrow$ & $\downarrow$ \\
\hline
\end{tabular}

Heat $=$ heat identification

Form $=$ product form

Test ID = identification of the test that generated the data

UnAged Treat $=$ heat treatment for sample without aging

AgeEnv $=$ aging environment for aged sample

T-Age $=$ aging temperature

t-Age $=$ aging time

TestEnv $=$ test environment 
ASSESSMENT OF EXISTING ALLOY 617 DATA FOR GEN IV NUCLEAR REACTOR APPLICATIONS

Table 5 (cont'd): Existing fatigue property data under various testing conditions

\begin{tabular}{|c|c|c|c|c|c|c|c|c|c|}
\hline $\mathbf{a}$ & $\begin{array}{c}\text { T-Test } \\
{ }^{\circ} \mathrm{C} \\
\end{array}$ & Control & Wave & $\mathbf{R}$ & $\begin{array}{c}\text { StnRng } \\
\% \\
\end{array}$ & $\begin{array}{c}\text { StsRng } \\
\text { MPa }\end{array}$ & $\begin{array}{c}\mathbf{f} \\
\mathbf{H z} \\
\end{array}$ & $\begin{array}{c}\text { StnRate } \\
1 / \text { sec } \\
\end{array}$ & $\mathbf{a}$ \\
\hline $\mathrm{b}$ & 850 & Stress & Sine & -1 & 0.27 & 413.7 & 0.5 & N/A & $\mathrm{b}$ \\
\hline $\mathrm{c}$ & 950 & Stress & Sine & -1 & 0.13 & 206.8 & 30 & N/A & $\mathrm{c}$ \\
\hline $\mathrm{d}$ & 950 & Stress & Sine & -1 & 0.17 & 248.2 & 30 & N/A & $\mathrm{d}$ \\
\hline $\mathrm{e}$ & 850 & Stress & Sine & -1 & 0.2 & 333.7 & 30 & $\mathrm{~N} / \mathrm{A}$ & $\mathrm{e}$ \\
\hline $\mathrm{f}$ & 950 & Stress & Sine & -1 & 0.24 & 317.2 & 30 & $\mathrm{~N} / \mathrm{A}$ & $\mathrm{f}$ \\
\hline $\mathrm{g}$ & 850 & Strain & Triangle & -1 & 0.4 & 572.3 & N/A & 0.4 & $\mathrm{~g}$ \\
\hline $\mathrm{h}$ & 950 & Strain & Triangle & -1 & 0.4 & 391.6 & $\mathrm{~N} / \mathrm{A}$ & 0.4 & $\mathrm{~h}$ \\
\hline $\mathrm{i}$ & 950 & Strain & Triangle & -1 & 1.0 & 375.1 & $\mathrm{~N} / \mathrm{A}$ & 0.4 & $\mathrm{i}$ \\
\hline $\mathrm{j}$ & 850 & Strain & Triangle & -1 & 0.3 & 435.8 & N/A & 0.4 & $\mathrm{j}$ \\
\hline $\mathrm{k}$ & 950 & Strain & Triangle & -1 & 1.0 & 404.0 & $\mathrm{~N} / \mathrm{A}$ & 0.4 & $\mathrm{k}$ \\
\hline 1 & 950 & Strain & Triangle & -1 & 1.0 & 441 & $\mathrm{~N} / \mathrm{A}$ & 0.4 & 1 \\
\hline $\mathrm{m}$ & 950 & Strain & Triangle & -1 & 0.25 & 368 & $\mathrm{~N} / \mathrm{A}$ & 0.05 & $\mathrm{~m}$ \\
\hline $\mathrm{n}$ & 950 & Strain & Triangle & -1 & 1.0 & 510 & $\mathrm{~N} / \mathrm{A}$ & 0.4 & $\mathrm{n}$ \\
\hline $\mathrm{O}$ & 950 & Strain & Triangle & -1 & 0.25 & 354 & $\mathrm{~N} / \mathrm{A}$ & 0.05 & $\mathrm{O}$ \\
\hline $\mathrm{p}$ & 950 & Strain & Trapezoidal & -1 & 1.0 & 352 & $\mathrm{~N} / \mathrm{A}$ & 0.05 & $\mathrm{p}$ \\
\hline $\mathrm{q}$ & 950 & Strain & Trapezoidal & -1 & 0.6 & 372 & $\mathrm{~N} / \mathrm{A}$ & 0.1 & $\mathrm{q}$ \\
\hline $\mathrm{r}$ & 950 & Strain & Trapezoidal & -1 & 0.4 & 374 & $\mathrm{~N} / \mathrm{A}$ & 0.08 & $\mathrm{r}$ \\
\hline $\mathrm{S}$ & 950 & Strain & Trapezoidal & -1 & 0.6 & 363 & $\mathrm{~N} / \mathrm{A}$ & 0.1 & $\mathrm{~S}$ \\
\hline$\downarrow$ & $\downarrow$ & $\downarrow$ & $\downarrow$ & $\downarrow$ & $\downarrow$ & $\downarrow$ & $\downarrow$ & $\downarrow$ & $\downarrow$ \\
\hline
\end{tabular}

T-Test $=$ test temperature

Control $=$ control signal of fatigue test

Wave $=$ waveform of fatigue control signal

$\mathbf{R}=$ minimum stress or strain / maximum stress or strain

StnRng $=$ strain range

StsRng $=$ stress range

$\mathbf{f}=$ frequency

StnRate $=$ strain rate 
ASSESSMENT OF EXISTING ALLOY 617 DATA FOR GEN IV NUCLEAR REACTOR APPLICATIONS

Table 5 (cont'd): Existing fatigue property data under various testing conditions

\begin{tabular}{|c|c|c|c|c|c|c|}
\hline $\mathbf{a}$ & $\begin{array}{c}\text { t-Hold } \\
\text { sec }\end{array}$ & $\begin{array}{l}\text { N-Sep } \\
\text { Cycle }\end{array}$ & $\begin{array}{c}\text { N-5\%StsR } \\
\text { Decrease }\end{array}$ & $\begin{array}{c}\text { N-50\%StsR } \\
\text { Decrease }\end{array}$ & Source & $\mathbf{a}$ \\
\hline $\mathrm{b}$ & 0 & $2.80 \mathrm{E}+07$ & $\mathrm{~N} / \mathrm{A}$ & $\mathrm{N} / \mathrm{A}$ & Baldwin0486T8 & $\mathrm{b}$ \\
\hline $\mathrm{c}$ & 0 & $1.90 \mathrm{E}+06$ & N/A & $\mathrm{N} / \mathrm{A}$ & Baldwin0486T8 & $\mathrm{c}$ \\
\hline $\mathrm{d}$ & 0 & 961000 & $\mathrm{~N} / \mathrm{A}$ & $\mathrm{N} / \mathrm{A}$ & Baldwin0486T8 & $\mathrm{d}$ \\
\hline $\mathrm{e}$ & 0 & $1.2 \mathrm{E}+06$ & $\mathrm{~N} / \mathrm{A}$ & $\mathrm{N} / \mathrm{A}$ & Baldwin0486T8 & $\mathrm{e}$ \\
\hline $\mathrm{f}$ & 0 & 42800 & $\mathrm{~N} / \mathrm{A}$ & $\mathrm{N} / \mathrm{A}$ & Baldwin0486T8 & $\mathrm{f}$ \\
\hline $\mathrm{g}$ & 0 & - & 3100 & 3685 & Baldwin0486T9 & $\mathrm{g}$ \\
\hline $\mathrm{h}$ & 0 & - & 5900 & 7153 & Baldwin0486T9 & $\mathrm{h}$ \\
\hline $\mathrm{i}$ & 0 & - & 1000 & 1423 & Baldwin0486T9 & $\mathrm{i}$ \\
\hline $\mathrm{j}$ & 0 & - & 16300 & 18997 & Baldwin0486T9 & $\mathrm{j}$ \\
\hline $\mathrm{k}$ & 0 & - & 940 & 985 & Baldwin0486T9 & $\mathrm{k}$ \\
\hline 1 & 0 & - & 590 & - & Baldwin0486T10 & 1 \\
\hline $\mathrm{m}$ & 0 & - & 19000 & - & Baldwin0486T10 & $\mathrm{m}$ \\
\hline $\mathrm{n}$ & 0 & - & 1615 & - & Baldwin0486T10 & $\mathrm{n}$ \\
\hline $\mathrm{O}$ & 0 & - & 47000 & - & Baldwin0486T10 & $\mathrm{O}$ \\
\hline $\mathrm{p}$ & 120 & - & 420 & - & Baldwin0486T11 & $\mathrm{p}$ \\
\hline $\mathrm{q}$ & 120 & - & 850 & - & Baldwin0486T11 & $\mathrm{q}$ \\
\hline $\mathrm{r}$ & 120 & - & 2000 & - & Baldwin0486T11 & $\mathrm{r}$ \\
\hline $\mathrm{S}$ & 1200 & - & 610 & - & Baldwin0486T11 & $\mathrm{S}$ \\
\hline$\downarrow$ & $\downarrow$ & $\downarrow$ & $\downarrow$ & $\downarrow$ & $\downarrow$ & $\downarrow$ \\
\hline
\end{tabular}

t-Hold $=$ hold time

$\mathbf{N}$-Sep $=$ number of cycles to total separation

$\mathbf{N}-\mathbf{5} \%$ StsR $=$ number of cycles to a $5 \%$ decrease in the stress range below the saturation value

$\mathbf{N}-\mathbf{5 0} \% \mathrm{StsR}=$ number of cycles to a $50 \%$ decrease in the stress range below the saturation value

Source $=$ the tag indicating data source as discussed in Section 2.2 
Table 5 (cont'd): Existing fatigue property data under various testing conditions

\begin{tabular}{|c|c|l|}
\hline a & DC & Note \\
\hline b & 4 & Extensometer for 300 cycles \\
\hline c & 4 & \\
\hline d & & \\
\hline e & 4 & \\
\hline f & 4 & \\
\hline g & & \\
\hline h & 4 & StsRng value at $50 \% \mathrm{StsR} / 2$ \\
\hline i & 4 & StsRng value at $50 \% \mathrm{StsR} / 2$ \\
\hline $\mathrm{j}$ & 4 & StsRng value at $50 \% \mathrm{StsR} / 2$ \\
\hline $\mathrm{k}$ & 4 & StsRng value at $5 \% \mathrm{StsR} / 2$ \\
\hline l & & \\
\hline $\mathrm{m}$ & 4 & StsRng value at $5 \% \mathrm{StsR} / 2$ \\
\hline $\mathrm{q}$ & 4 & StsRng value at $5 \% \mathrm{StsR} / 2$ \\
\hline $\mathrm{n}$ & 4 & StsRng value at $5 \% \mathrm{StsR} / 2$ \\
\hline $\mathrm{o}$ & 4 & StsRng value at $5 \% \mathrm{StsR} / 2$ \\
\hline $\mathrm{p}$ & & \\
\hline $\mathrm{r}$ & 4 & StsRng value at $5 \% \mathrm{StsR} / 2$ \\
\hline $\mathrm{s}$ & 4 & StsRng value at $5 \% \mathrm{StsR} / 2$ \\
\hline & 4 & StsRng value at $5 \% \mathrm{StsR} / 2$ \\
\hline
\end{tabular}

DC $=$ data classification as discussed in Section 2.1

Note $=$ comments or explanations for the data point of the test 
Table 6: Existing toughness property data under various testing conditions

\begin{tabular}{|c|c|c|c|c|c|c|c|}
\hline Heat & Form & $\begin{array}{c}\text { UnAged } \\
\text { Treat }\end{array}$ & AgeEnv & $\begin{array}{c}\text { T-Age } \\
{ }^{\circ} \mathrm{C}\end{array}$ & $\begin{array}{c}\text { t-Age } \\
\text { h }\end{array}$ & $\underset{\mathbf{J}}{\text { E-CharpyV }}$ & $\mathbf{a}$ \\
\hline XX01A3US & $13 \mathrm{~mm}$ plate & $\begin{array}{l}\text { solution } \\
\text { annealed }\end{array}$ & $\mathrm{N} / \mathrm{A}$ & $\mathrm{N} / \mathrm{A}$ & 0 & 175 & $\mathrm{~b}$ \\
\hline XX01A3US & $13 \mathrm{~mm}$ plate & $\begin{array}{c}\text { solution } \\
\text { annealed }\end{array}$ & N/A & N/A & 0 & 175 & $\mathrm{c}$ \\
\hline XX01A3US & $13 \mathrm{~mm}$ plate & $\begin{array}{c}\text { solution } \\
\text { annealed }\end{array}$ & N/A & $\mathrm{N} / \mathrm{A}$ & 0 & 175 & $\mathrm{~d}$ \\
\hline XX01A3US & $13 \mathrm{~mm}$ plate & $\begin{array}{c}\text { solution } \\
\text { annealed }\end{array}$ & N/A & N/A & 0 & 175 & $\mathrm{e}$ \\
\hline XX09A4UK & $9.5 \mathrm{~mm}$ plate & $\begin{array}{l}\text { solution } \\
\text { annealed }\end{array}$ & $\mathrm{N} / \mathrm{A}$ & $\mathrm{N} / \mathrm{A}$ & 0 & 298 & $\mathrm{f}$ \\
\hline XX01A3US & $13 \mathrm{~mm}$ plate & $\mathrm{N} / \mathrm{A}$ & impure $\mathrm{He}$ & 871 & 100 & 91 & $\mathrm{~g}$ \\
\hline XX01A3US & $13 \mathrm{~mm}$ plate & N/A & inert & 871 & 100 & 98 & $\mathrm{~h}$ \\
\hline XX09A4UK & $9.5 \mathrm{~mm}$ plate & $\mathrm{N} / \mathrm{A}$ & impure $\mathrm{He}$ & 871 & 100 & 61 & $\mathrm{i}$ \\
\hline XX01A3US & $13 \mathrm{~mm}$ plate & $\mathrm{N} / \mathrm{A}$ & inert & 871 & 1000 & 53 & $\mathrm{j}$ \\
\hline XX09A4UK & $9.5 \mathrm{~mm}$ plate & $\mathrm{N} / \mathrm{A}$ & inert & 871 & 1000 & 26 & $\mathrm{k}$ \\
\hline XX01A3US & $13 \mathrm{~mm}$ plate & $\mathrm{N} / \mathrm{A}$ & inert & 538 & 10000 & 81 & 1 \\
\hline XX01A3US & $13 \mathrm{~mm}$ plate & $\mathrm{N} / \mathrm{A}$ & inert & 704 & 10000 & 31 & $\mathrm{~m}$ \\
\hline XX01A3US & $13 \mathrm{~mm}$ plate & $\mathrm{N} / \mathrm{A}$ & inert & 871 & 10000 & 26 & $\mathrm{n}$ \\
\hline XX01A3US & $13 \mathrm{~mm}$ plate & $\mathrm{N} / \mathrm{A}$ & inert & 538 & 20000 & 103 & 0 \\
\hline XX01A3US & $13 \mathrm{~mm}$ plate & $\mathrm{N} / \mathrm{A}$ & inert & 704 & 20000 & 20 & $\mathrm{p}$ \\
\hline XX01A3US & $13 \mathrm{~mm}$ plate & $\mathrm{N} / \mathrm{A}$ & inert & 871 & 20000 & 20 & $q$ \\
\hline XX01A3US & $13 \mathrm{~mm}$ plate & N/A & inert & 492 & 28300 & 190 & $r$ \\
\hline$\downarrow$ & $\downarrow$ & $\downarrow$ & $\downarrow$ & $\downarrow$ & $\downarrow$ & $\downarrow$ & $\downarrow$ \\
\hline
\end{tabular}

Heat $=$ heat identification

Form $=$ product form

UnAged Treat $=$ heat treatment for sample without aging

AgeEnv $=$ aging environment for aged sample

T-Age $=$ aging temperature

t-Age $=$ aging time

E-CharpyV = Charpy V impact energy 
Table 6 (cont'd): Existing toughness property data under various testing conditions

\begin{tabular}{|c|c|c|c|}
\hline $\mathbf{a}$ & Source & DC & Note \\
\hline $\mathrm{b}$ & McCoy0285T13 & 5 & \\
\hline $\mathrm{c}$ & McCoy0285T13 & 5 & \\
\hline $\mathrm{d}$ & McCoy0285T13 & 5 & \\
\hline $\mathrm{e}$ & McCoy0285T13 & 5 & \\
\hline $\mathrm{f}$ & McCoy0285T13 & 5 & \\
\hline $\mathrm{g}$ & McCoy0285T13 & 5 & \\
\hline $\mathrm{h}$ & McCoy0285T13 & 5 & $\begin{array}{l}\text { sample machined after } \\
\text { block aged in air }\end{array}$ \\
\hline $\mathrm{i}$ & McCoy0285T13 & 5 & \\
\hline $\mathrm{j}$ & McCoy0285T13 & 5 & $\begin{array}{l}\text { sample machined after } \\
\text { block aged in air }\end{array}$ \\
\hline $\mathrm{k}$ & McCoy0285T13 & 5 & $\begin{array}{l}\text { sample machined after } \\
\text { block aged in air }\end{array}$ \\
\hline 1 & McCoy0285T13 & 5 & $\begin{array}{l}\text { sample machined after } \\
\text { block aged in air }\end{array}$ \\
\hline $\mathrm{m}$ & McCoy0285T13 & 5 & $\begin{array}{l}\text { sample machined after } \\
\text { block aged in air }\end{array}$ \\
\hline $\mathrm{n}$ & McCoy0285T13 & 5 & $\begin{array}{l}\text { sample machined after } \\
\text { block aged in air }\end{array}$ \\
\hline o & McCoy0285T13 & 5 & $\begin{array}{l}\text { sample machined after } \\
\text { block aged in air }\end{array}$ \\
\hline $\mathrm{p}$ & McCoy0285T13 & 5 & $\begin{array}{l}\text { sample machined after } \\
\text { block aged in air }\end{array}$ \\
\hline$q$ & McCoy0285T13 & 5 & $\begin{array}{l}\text { sample machined after } \\
\text { block aged in air }\end{array}$ \\
\hline $\mathrm{r}$ & McCoy0285T13 & 5 & $\begin{array}{l}\text { sample machined after } \\
\text { block aged in steam }\end{array}$ \\
\hline$\downarrow$ & $\downarrow$ & $\downarrow$ & $\downarrow$ \\
\hline
\end{tabular}

Source $=$ the tag indicating data source as discussed in Section 2.2

DC $=$ data classification as discussed in Section 2.1

Note $=$ comments or explanations for the data point of the test 
ASSESSMENT OF EXISTING ALLOY 617 DATA FOR GEN IV NUCLEAR REACTOR APPLICATIONS

Table 7: Number of existing tensile property data and testing conditions

\begin{tabular}{|c|c|c|c|c|c|c|c|}
\hline $\begin{array}{c}\text { T-Test } \\
{ }^{\circ} \mathrm{C}\end{array}$ & $\begin{array}{c}\text { UnAged } \\
\text { Treat }\end{array}$ & AgeEnv & $\begin{array}{c}\text { T-Age } \\
{ }^{\circ} \mathrm{C}\end{array}$ & $\begin{array}{c}\text { t-Age } \\
h\end{array}$ & $\begin{array}{c}\mathbf{E} \\
\text { Data }\end{array}$ & $\begin{array}{c}\text { YS } \\
\text { Data }\end{array}$ & $\mathbf{a}$ \\
\hline 22 & $\mathrm{~N} / \mathrm{A}$ & inert gas & 538 & 2,500 & 0 & 2 & $\mathrm{~b}$ \\
\hline 22 & $\mathrm{~N} / \mathrm{A}$ & inert gas & 538 & 20000 & 0 & 1 & $\mathrm{c}$ \\
\hline 22 & $\mathrm{~N} / \mathrm{A}$ & inert gas & 704 & 2,500 & 0 & 2 & $\mathrm{~d}$ \\
\hline 22 & $\mathrm{~N} / \mathrm{A}$ & inert gas & 704 & 20000 & 0 & 1 & $\mathrm{e}$ \\
\hline 22 & $\mathrm{~N} / \mathrm{A}$ & inert gas & 871 & 2,500 & 0 & 2 & $\mathrm{f}$ \\
\hline 22 & $\mathrm{~N} / \mathrm{A}$ & inert gas & 871 & 20000 & 0 & 1 & $\mathrm{~g}$ \\
\hline 22 & $\begin{array}{c}\text { solution } \\
\text { annealed }\end{array}$ & N/A & N/A & N/A & 0 & 3 & $\mathrm{~h}$ \\
\hline 24 & $\mathrm{~N} / \mathrm{A}$ & impure $\mathrm{He}$ & 593 & 20000 & 0 & 1 & $\bar{i}$ \\
\hline 24 & $\mathrm{~N} / \mathrm{A}$ & impure $\mathrm{He}$ & 704 & 20000 & 0 & 1 & $\mathrm{j}$ \\
\hline 24 & $\mathrm{~N} / \mathrm{A}$ & impure $\mathrm{He}$ & 871 & 20000 & 0 & 1 & $\mathrm{k}$ \\
\hline 25 & $\mathrm{~N} / \mathrm{A}$ & impure $\mathrm{He}$ & 871 & 26117 & 0 & 1 & 1 \\
\hline 25 & N/A & inert & 482 & 28300 & 0 & 1 & $\mathrm{~m}$ \\
\hline 25 & $\mathrm{~N} / \mathrm{A}$ & inert & 538 & 28300 & 0 & 1 & $\mathrm{n}$ \\
\hline 25 & unknown & N/A & $\mathrm{N} / \mathrm{A}$ & 0 & 0 & 2 & 0 \\
\hline 538 & N/A & inert gas & 538 & 2500 & 0 & 2 & $\mathrm{p}$ \\
\hline 538 & $\mathrm{~N} / \mathrm{A}$ & inert gas & 538 & 10000 & 0 & 2 & $q$ \\
\hline 538 & $\mathrm{~N} / \mathrm{A}$ & inert gas & 538 & 20000 & 0 & 2 & $\mathrm{r}$ \\
\hline 538 & $\mathrm{~N} / \mathrm{A}$ & inert gas & 704 & 10000 & 0 & 2 & $\mathrm{~s}$ \\
\hline 538 & $\begin{array}{l}\text { solution } \\
\text { annealed }\end{array}$ & $\mathrm{N} / \mathrm{A}$ & N/A & 0 & 0 & 2 & $\mathrm{t}$ \\
\hline 593 & N/A & impure $\mathrm{He}$ & 593 & 20000 & 0 & 1 & $\mathrm{u}$ \\
\hline 593 & unknown & $\mathrm{N} / \mathrm{A}$ & $\mathrm{N} / \mathrm{A}$ & 0 & 0 & 1 & $\mathrm{~V}$ \\
\hline 649 & unknown & $\mathrm{N} / \mathrm{A}$ & N/A & 0 & 0 & 1 & $\mathrm{~W}$ \\
\hline 704 & N/A & impure $\mathrm{He}$ & 704 & 20000 & 0 & 1 & $\mathrm{x}$ \\
\hline 704 & $\mathrm{~N} / \mathrm{A}$ & inert gas & 704 & 2500 & 0 & 2 & $\mathrm{y}$ \\
\hline 704 & $\mathrm{~N} / \mathrm{A}$ & inert gas & 704 & 20000 & 0 & 1 & $\mathrm{Z}$ \\
\hline 704 & $\begin{array}{l}\text { solution } \\
\text { annealed }\end{array}$ & N/A & N/A & 0 & 0 & 3 & a1 \\
\hline 760 & unknown & $\mathrm{N} / \mathrm{A}$ & $\mathrm{N} / \mathrm{A}$ & 0 & 0 & 1 & $\mathrm{~b} 2$ \\
\hline 871 & $\mathrm{~N} / \mathrm{A}$ & impure $\mathrm{He}$ & 871 & 20000 & 0 & 1 & $\mathrm{c} 3$ \\
\hline$\downarrow$ & $\downarrow$ & $\downarrow$ & $\downarrow$ & $\downarrow$ & $\downarrow$ & $\downarrow$ & $\downarrow$ \\
\hline
\end{tabular}

T-Test $=$ test temperature

UnAged Treat $=$ heat treatment for sample without aging

AgeEnv = aging environment for aged sample

T-Age $=$ aging temperature

t-Age $=$ aging time

$\mathbf{E}=$ Young's modulurs

$\mathbf{Y S}=$ yield stress 
Table 7 (cont'd): Number of existing tensile property data and testing conditions

\begin{tabular}{|c|c|c|c|c|c|}
\hline $\mathbf{a}$ & $\begin{array}{l}\text { UTS } \\
\text { Data }\end{array}$ & $\begin{array}{c}\text { UnifStn } \\
\text { Data }\end{array}$ & $\begin{array}{c}\text { TotalStn } \\
\text { Data }\end{array}$ & $\begin{array}{c}\text { RA } \\
\text { Data }\end{array}$ & DC \\
\hline $\mathrm{b}$ & 2 & 2 & 2 & 2 & 5 \\
\hline $\mathrm{c}$ & 1 & 1 & 1 & 1 & 5 \\
\hline $\mathrm{d}$ & 2 & 2 & 2 & 2 & 5 \\
\hline $\mathrm{e}$ & 1 & 1 & 1 & 1 & 5 \\
\hline $\mathrm{f}$ & 2 & 2 & 2 & 2 & 5 \\
\hline $\mathrm{g}$ & 1 & 1 & 1 & 1 & 5 \\
\hline $\mathrm{h}$ & 3 & 3 & 3 & 3 & 5 \\
\hline $\mathrm{i}$ & 1 & 1 & 1 & 1 & 5 \\
\hline $\mathrm{j}$ & 1 & 1 & 1 & 1 & 5 \\
\hline $\mathrm{k}$ & 1 & 1 & 1 & 1 & 5 \\
\hline 1 & 1 & 1 & 1 & 1 & 5 \\
\hline $\mathrm{m}$ & 1 & 1 & 1 & 1 & 5 \\
\hline $\mathrm{n}$ & 1 & 1 & 1 & 1 & 5 \\
\hline 0 & 2 & 2 & 2 & 2 & 5 \\
\hline $\mathrm{p}$ & 2 & 2 & 2 & 2 & 5 \\
\hline $\mathrm{q}$ & 2 & 2 & 2 & 2 & 5 \\
\hline$r$ & 2 & 2 & 2 & 2 & 5 \\
\hline $\mathrm{S}$ & 2 & 2 & 2 & 2 & 5 \\
\hline $\mathrm{t}$ & 2 & 2 & 2 & 2 & 5 \\
\hline $\mathrm{u}$ & 1 & 1 & 1 & 1 & 5 \\
\hline $\mathrm{V}$ & 1 & 1 & 1 & 1 & 5 \\
\hline $\mathrm{W}$ & 1 & 1 & 1 & 1 & 5 \\
\hline $\mathrm{X}$ & 1 & 1 & 1 & 1 & 5 \\
\hline $\mathrm{y}$ & 2 & 2 & 1 & 1 & 5 \\
\hline $\mathrm{Z}$ & 1 & 1 & 1 & 1 & 5 \\
\hline a1 & 3 & 3 & 3 & 3 & 5 \\
\hline b2 & 1 & 1 & 1 & 1 & 5 \\
\hline c3 & 1 & 1 & 1 & 1 & 5 \\
\hline$\downarrow$ & $\downarrow$ & $\downarrow$ & $\downarrow$ & $\downarrow$ & $\downarrow$ \\
\hline
\end{tabular}

UTS $=$ ultimate tensile stress

UnifStn = uniform strain, the strain prior to necking

TotalStn $=$ total strain, the strain at rupture

$\mathbf{R A}=$ area of reduction

DC $=$ data classification as discussed in Section 2.1 
Table 8: Number of existing weld tensile property data and testing conditions

\begin{tabular}{|c|c|c|c|c|c|c|}
\hline $\begin{array}{c}\text { BM } \\
\text { Treat }\end{array}$ & WM & $\begin{array}{l}\text { Sample } \\
\text { Content }\end{array}$ & $\begin{array}{c}\text { SampleNoAge } \\
\text { Treat } \\
\end{array}$ & $\begin{array}{l}\text { Sample } \\
\text { AgeEnv }\end{array}$ & $\begin{array}{c}\text { T-SampleAge } \\
{ }^{\circ} \mathrm{C} \\
\end{array}$ & $\mathbf{a}$ \\
\hline $\begin{array}{l}\text { solution } \\
\text { annealed }\end{array}$ & GTA & W & as welded & N/A & N/A & $\mathrm{b}$ \\
\hline $\begin{array}{c}\text { solution } \\
\text { annealed }\end{array}$ & GTA & W & as welded & N/A & N/A & $\mathrm{c}$ \\
\hline $\begin{array}{l}\text { solution } \\
\text { annealed }\end{array}$ & GTA & W & as welded & N/A & N/A & $\mathrm{d}$ \\
\hline $\begin{array}{l}\text { solution } \\
\text { annealed }\end{array}$ & GTA & W & as welded & N/A & N/A & e \\
\hline $\begin{array}{l}\text { solution } \\
\text { annealed }\end{array}$ & GTA & W & as welded & N/A & N/A & $\mathrm{f}$ \\
\hline $\begin{array}{c}\text { solution } \\
\text { annealed }\end{array}$ & GTA & BWB & N/A & impure $\mathrm{He}$ & 593 & $\mathrm{~g}$ \\
\hline $\begin{array}{c}\text { solution } \\
\text { annealed }\end{array}$ & GTA & BWB & N/A & impure $\mathrm{He}$ & 593 & $\mathrm{~h}$ \\
\hline $\begin{array}{c}\text { solution } \\
\text { annealed }\end{array}$ & GTA & BWB & N/A & impure $\mathrm{He}$ & 704 & $\mathrm{i}$ \\
\hline $\begin{array}{c}\text { solution } \\
\text { annealed }\end{array}$ & GTA & BWB & N/A & impure $\mathrm{He}$ & 704 & $\mathrm{j}$ \\
\hline $\begin{array}{c}\text { solution } \\
\text { annealed }\end{array}$ & GTA & BWB & N/A & impure $\mathrm{He}$ & 871 & $\mathrm{k}$ \\
\hline $\begin{array}{c}\text { solution } \\
\text { annealed }\end{array}$ & GTA & BWB & N/A & impure $\mathrm{He}$ & 593 & 1 \\
\hline $\begin{array}{c}\text { solution } \\
\text { annealed }\end{array}$ & GTA & BWB & N/A & impure $\mathrm{He}$ & 704 & $\mathrm{~m}$ \\
\hline $\begin{array}{c}\text { solution } \\
\text { annealed }\end{array}$ & GTA & BWB & N/A & impure $\mathrm{He}$ & 871 & $\mathrm{n}$ \\
\hline$\downarrow$ & $\downarrow$ & $\downarrow$ & $\downarrow$ & $\downarrow$ & $\downarrow$ & $\downarrow$ \\
\hline
\end{tabular}

BM Treat $=$ heat treatment of the base metal

$\mathbf{W M}=$ welding method to produce the sample weld: GTA $=$ gas tungsten arc welding Sample Content $=$ composition of the sample: $\mathrm{W}=$ weld metal, $\mathrm{B}=$ base metal, $\mathrm{BWB}=$ weldment, the sample was machined across the weld to include the weld in the middle and base metal on both sides

SampleNoAge Treat $=$ heat treatment of sample that has not been aged

Sample AgeEnv = aging environment of aged sample

T-SampleAge $=$ sample aging temperature 
ASSESSMENT OF EXISTING ALLOY 617 DATA FOR GEN IV NUCLEAR REACTOR APPLICATIONS

Table 8 (cont'd): Number of weld existing tensile property data and testing conditions

\begin{tabular}{|c|c|c|c|c|c|c|c|}
\hline $\mathbf{a}$ & $\begin{array}{c}\text { t-SampleAge } \\
\text { H } \\
\end{array}$ & $\begin{array}{c}\text { T-Test } \\
{ }^{\circ} \mathrm{C} \\
\end{array}$ & $\begin{array}{c}0.2 \% Y S \\
\text { Data }\end{array}$ & $\begin{array}{l}\text { UTS } \\
\text { Data }\end{array}$ & $\begin{array}{c}\text { UnifStn } \\
\text { Data }\end{array}$ & $\begin{array}{c}\text { TotalStn } \\
\text { Data }\end{array}$ & $\begin{array}{c}\text { RA } \\
\text { Data }\end{array}$ \\
\hline $\mathrm{b}$ & 0 & 25 & 1 & 1 & 1 & 1 & 1 \\
\hline $\mathrm{c}$ & 0 & 593 & 1 & 1 & 1 & 1 & 1 \\
\hline $\mathrm{d}$ & 0 & 649 & 1 & 1 & 1 & 1 & 1 \\
\hline $\mathrm{e}$ & 0 & 760 & 1 & 1 & 1 & 1 & 1 \\
\hline $\mathrm{f}$ & 0 & 871 & 1 & 1 & 1 & 1 & 1 \\
\hline $\mathrm{g}$ & 10,000 & 24 & 1 & 1 & 1 & 1 & 1 \\
\hline $\mathrm{h}$ & 20,000 & 24 & 1 & 1 & 1 & 1 & 1 \\
\hline $\mathrm{i}$ & 10,000 & 24 & 1 & 1 & 1 & 1 & 1 \\
\hline $\mathrm{j}$ & 20,000 & 24 & 1 & 1 & 1 & 1 & 1 \\
\hline $\mathrm{k}$ & 20,000 & 24 & 1 & 1 & 1 & 1 & 1 \\
\hline 1 & 20,000 & 593 & 1 & 1 & 1 & 1 & 1 \\
\hline $\mathrm{m}$ & 20,000 & 704 & 1 & 1 & 1 & 1 & 1 \\
\hline $\mathrm{n}$ & 20,000 & 871 & 1 & 1 & 1 & 1 & 1 \\
\hline & $\downarrow$ & $\downarrow$ & $\downarrow$ & $\downarrow$ & $\downarrow$ & $\downarrow$ & $\downarrow$ \\
\hline
\end{tabular}

t-SampleAge $=$ sample aging time

T-Test $=$ test temperature

$0.2 \% \mathrm{YS}=0.2 \%$ yield stress

UTS $=$ ultimate tensile stress

UnifStn = uniform strain, the strain prior to necking

TotalStn $=$ total strain, the strain at rupture

$\mathbf{R A}=$ area of reduction 
Table 9: Number of existing creep property data and testing conditions

\begin{tabular}{|c|c|c|c|c|c|c|c|c|}
\hline $\begin{array}{c}\text { TestT } \\
{ }^{\circ} \mathrm{C} \\
\end{array}$ & $\begin{array}{c}\text { TestSts } \\
\text { MPa } \\
\end{array}$ & TestEnv & $\begin{array}{c}\text { UnAged } \\
\text { Treat } \\
\end{array}$ & AgeEnv & $\begin{array}{c}\text { T-Age } \\
{ }^{\circ} \mathrm{C} \\
\end{array}$ & $\begin{array}{c}\text { t-Age } \\
\text { h } \\
\end{array}$ & $\begin{array}{c}\text { t-1\%Stn } \\
\text { Data } \\
\end{array}$ & $\mathbf{a}$ \\
\hline 593 & 345 & Air & $\begin{array}{l}\text { solution } \\
\text { annealed }\end{array}$ & $\mathrm{N} / \mathrm{A}$ & N/A & N/A & 0 & $\mathrm{~b}$ \\
\hline 593 & 345 & $\mathrm{He}$ & $\mathrm{N} / \mathrm{A}$ & impure He & 593 & 10,000 & 0 & $\mathrm{c}$ \\
\hline 593 & 414 & Air & $\begin{array}{c}\text { solution } \\
\text { annealed }\end{array}$ & $\mathrm{N} / \mathrm{A}$ & N/A & $\mathrm{N} / \mathrm{A}$ & 1 & d \\
\hline 593 & 414 & $\mathrm{He}$ & $\begin{array}{c}\text { solution } \\
\text { annealed }\end{array}$ & N/A & N/A & $\mathrm{N} / \mathrm{A}$ & 1 & e \\
\hline 593 & 414 & $\mathrm{He}$ & N/A & impure $\mathrm{He}$ & 593 & 10,000 & 1 & $\mathrm{f}$ \\
\hline 593 & 414 & $\mathrm{He}$ & N/A & impure $\mathrm{He}$ & 593 & 20,000 & 1 & $\mathrm{~g}$ \\
\hline 649 & 207 & $\mathrm{He}$ & $\begin{array}{c}\text { solution } \\
\text { annealed }\end{array}$ & $\mathrm{N} / \mathrm{A}$ & N/A & $\mathrm{N} / \mathrm{A}$ & 0 & $\mathrm{~h}$ \\
\hline 649 & 276 & Air & $\begin{array}{c}\text { solution } \\
\text { annealed }\end{array}$ & $\mathrm{N} / \mathrm{A}$ & N/A & $\mathrm{N} / \mathrm{A}$ & 1 & $\mathrm{i}$ \\
\hline 649 & 276 & $\mathrm{He}$ & $\begin{array}{c}\text { solution } \\
\text { annealed }\end{array}$ & $\mathrm{N} / \mathrm{A}$ & $\mathrm{N} / \mathrm{A}$ & $\mathrm{N} / \mathrm{A}$ & 2 & $\mathrm{j}$ \\
\hline 649 & 276 & $\mathrm{He}$ & $\begin{array}{l}\text { solution } \\
\text { annealed }\end{array}$ & N/A & N/A & $\mathrm{N} / \mathrm{A}$ & 0 & $\mathrm{k}$ \\
\hline 649 & 345 & Air & N/A & inert & 482 & 28,300 & 1 & 1 \\
\hline 649 & 345 & Air & N/A & inert & 538 & 28,300 & 1 & $\mathrm{~m}$ \\
\hline 649 & 414 & $\mathrm{He}$ & $\begin{array}{c}\text { solution } \\
\text { annealed }\end{array}$ & $\mathrm{N} / \mathrm{A}$ & N/A & $\mathrm{N} / \mathrm{A}$ & 1 & $\mathrm{n}$ \\
\hline 704 & 172 & $\mathrm{He}$ & $\begin{array}{c}\text { solution } \\
\text { annealed }\end{array}$ & $\mathrm{N} / \mathrm{A}$ & N/A & N/A & 1 & o \\
\hline 704 & 207 & Air & $\begin{array}{c}\text { solution } \\
\text { annealed }\end{array}$ & N/A & N/A & $\mathrm{N} / \mathrm{A}$ & 0 & $\mathrm{p}$ \\
\hline 704 & 207 & $\mathrm{He}$ & N/A & impure He & 704 & 10,000 & 1 & $\mathrm{q}$ \\
\hline$\downarrow$ & $\downarrow$ & $\downarrow$ & $\downarrow$ & $\downarrow$ & $\downarrow$ & $\downarrow$ & $\downarrow$ & $\downarrow$ \\
\hline
\end{tabular}

T-Test $=$ test temperature

TestSts $=$ test stress

TestEnv $=$ test environment

UnAged Treat $=$ heat treatment for sample without aging

AgeEnv = aging environment for aged sample

T-Age $=$ aging temperature

t-Age $=$ aging time

t-1\%Stn = time for reaching $1 \%$ creep strain 
ASSESSMENT OF EXISTING ALLOY 617 DATA

FOR GEN IV NUCLEAR REACTOR APPLICATIONS

Table 9 (cont'd): Number of existing creep property data and testing conditions

\begin{tabular}{|c|c|c|c|c|c|c|c|c|c|}
\hline $\mathbf{a}$ & $\begin{array}{c}\text { t-2\%Stn } \\
\text { Data }\end{array}$ & $\begin{array}{c}\text { t-5\%Stn } \\
\text { Data }\end{array}$ & $\begin{array}{c}\text { t-TerStn } \\
\text { Data }\end{array}$ & $\begin{array}{l}\text { t-Rpt } \\
\text { Data }\end{array}$ & $\begin{array}{l}\text { MCR } \\
\text { Data }\end{array}$ & $\begin{array}{c}\text { LdgStn } \\
\text { Data }\end{array}$ & $\begin{array}{c}\text { CpStn } \\
\text { Data }\end{array}$ & $\begin{array}{c}\text { RA } \\
\text { Data }\end{array}$ & DC \\
\hline$b$ & 0 & 0 & 0 & 1 & 1 & 1 & 1 & 1 & 5 \\
\hline $\mathrm{c}$ & 0 & 0 & 0 & 1 & 1 & 1 & 1 & 1 & 5 \\
\hline & 0 & 1 & 1 & 1 & 1 & 1 & 1 & 1 & 5 \\
\hline $\mathrm{e}$ & 1 & 0 & 1 & 1 & 1 & 1 & 1 & 1 & 5 \\
\hline $\mathrm{f}$ & 0 & 0 & 0 & 1 & 1 & 0 & 1 & 1 & 5 \\
\hline $\mathrm{g}$ & 0 & 0 & 1 & 1 & 1 & 1 & 1 & 1 & 5 \\
\hline $\mathrm{h}$ & 0 & 0 & 0 & 1 & 1 & 1 & 1 & 0 & 5 \\
\hline$\overline{\mathrm{i}}$ & 1 & 1 & 1 & 3 & 3 & 3 & 3 & 3 & 5 \\
\hline $\bar{j}$ & 1 & 1 & 1 & 2 & 2 & 2 & 2 & 2 & 5 \\
\hline$\overline{\mathrm{k}}$ & 0 & 0 & 0 & 1 & 1 & 1 & 1 & 1 & 5 \\
\hline 1 & 1 & 0 & 1 & 1 & 1 & 1 & 1 & 1 & 5 \\
\hline $\mathrm{m}$ & 1 & 0 & 0 & 1 & 1 & 1 & 1 & 1 & 5 \\
\hline $\mathrm{n}$ & 1 & 1 & 1 & 1 & 1 & 1 & 1 & 1 & 5 \\
\hline o & 1 & 0 & 0 & 1 & 1 & 1 & 1 & 0 & 5 \\
\hline$p$ & 0 & 0 & 0 & 1 & 1 & 1 & 1 & 1 & 5 \\
\hline $\mathrm{q}$ & 1 & 0 & 1 & 1 & 1 & 0 & 1 & 1 & 5 \\
\hline$\downarrow$ & $\downarrow$ & $\downarrow$ & $\downarrow$ & $\downarrow$ & $\downarrow$ & $\downarrow$ & $\downarrow$ & $\downarrow$ & $\downarrow$ \\
\hline
\end{tabular}

$\mathbf{t}-\mathbf{2} \%$ Stn $=$ time for reaching $2 \%$ creep strain

$\mathbf{t}-\mathbf{5 \%}$ Stn $=$ time for reaching 5\% creep strain

$\mathbf{t}$-TerStn $=$ time for reaching tertiary creep strain

$\mathbf{t}-\mathbf{R p t}=$ creep rupture time

$\mathbf{M C R}=$ minimum creep rate

LdgStn $=$ loading strain

CpStn $=$ creep rupture strain

$\mathbf{R A}=$ area of reduction

DC $=$ data classification as discussed in Section 2.1 
Table 10: Number of existing weld creep property data and testing conditions

\begin{tabular}{|c|c|c|c|c|c|c|}
\hline BM Treat & WM & $\begin{array}{l}\text { Sample } \\
\text { Content }\end{array}$ & $\begin{array}{c}\text { SampleNoAge } \\
\text { Treat }\end{array}$ & $\begin{array}{l}\text { Sample } \\
\text { AgeEnv }\end{array}$ & $\begin{array}{c}\text { T-SampleAge } \\
{ }^{\circ} \mathrm{C} \\
\end{array}$ & $\mathbf{a}$ \\
\hline $\begin{array}{c}\text { solution } \\
\text { annealed }\end{array}$ & GTA & W & as welded & N/A & N/A & $\mathrm{b}$ \\
\hline $\begin{array}{l}\text { solution } \\
\text { annealed }\end{array}$ & GTA & W & as welded & $\mathrm{N} / \mathrm{A}$ & N/A & $\mathrm{c}$ \\
\hline $\begin{array}{l}\text { solution } \\
\text { annealed }\end{array}$ & GTA & W & as welded & N/A & N/A & $\mathrm{d}$ \\
\hline $\begin{array}{l}\text { solution } \\
\text { annealed }\end{array}$ & GTA & W & as welded & N/A & $\mathrm{N} / \mathrm{A}$ & $\mathrm{e}$ \\
\hline $\begin{array}{c}\text { solution } \\
\text { annealed }\end{array}$ & GTA & W & as welded & N/A & N/A & $\mathrm{f}$ \\
\hline $\begin{array}{c}\text { solution } \\
\text { annealed }\end{array}$ & GTA & W & as welded & N/A & N/A & $\mathrm{g}$ \\
\hline $\begin{array}{l}\text { solution } \\
\text { annealed }\end{array}$ & GTA & W & as welded & $\mathrm{N} / \mathrm{A}$ & $\mathrm{N} / \mathrm{A}$ & $\mathrm{h}$ \\
\hline $\begin{array}{l}\text { solution } \\
\text { annealed }\end{array}$ & GTA & W & as welded & $\mathrm{N} / \mathrm{A}$ & N/A & $\mathrm{i}$ \\
\hline $\begin{array}{c}\text { solution } \\
\text { annealed }\end{array}$ & GTA & W & as welded & N/A & N/A & $\mathrm{j}$ \\
\hline $\begin{array}{l}\text { solution } \\
\text { annealed }\end{array}$ & GTA & BWB & $\mathrm{N} / \mathrm{A}$ & impure $\mathrm{He}$ & 593 & $\mathrm{k}$ \\
\hline $\begin{array}{l}\text { solution } \\
\text { annealed }\end{array}$ & GTA & BWB & $\mathrm{N} / \mathrm{A}$ & impure $\mathrm{He}$ & 704 & 1 \\
\hline $\begin{array}{c}\text { solution } \\
\text { annealed }\end{array}$ & GTA & BWB & $\mathrm{N} / \mathrm{A}$ & impure He & 704 & $\mathrm{~m}$ \\
\hline$\downarrow$ & $\downarrow$ & $\downarrow$ & $\downarrow$ & $\downarrow$ & $\downarrow$ & $\downarrow$ \\
\hline
\end{tabular}

BM Treat $=$ heat treatment of the base metal

$\mathbf{W M}=$ welding method to produce the sample weld: GTA = gas tungsten arc welding Sample Content $=$ composition of the sample: $\mathrm{W}=$ weld metal, $\mathrm{B}=$ base metal, $\mathrm{BWB}=$ weldment, the sample was machined across the weld to include the weld in the middle and base metal on both sides

SampleNoAge Treat $=$ heat treatment of sample that has not been aged

Sample AgeEnv = aging environment of aged sample

T-SampleAge $=$ sample aging temperature 
ASSESSMENT OF EXISTING ALLOY 617 DATA FOR GEN IV NUCLEAR REACTOR APPLICATIONS

Table 10 (cont'd): Number of weld existing creep property data and testing conditions

\begin{tabular}{|c|c|c|c|c|c|c|c|c|}
\hline $\mathbf{a}$ & $\begin{array}{c}\text { t-SampleAge } \\
\text { H }\end{array}$ & $\begin{array}{c}\text { T-Test } \\
{ }^{\circ} \mathrm{C}\end{array}$ & $\begin{array}{c}\text { TestSts } \\
\text { MPa }\end{array}$ & $\begin{array}{l}\text { Test } \\
\text { Env }\end{array}$ & $\begin{array}{c}\text { t-1\%Stn } \\
\text { Data }\end{array}$ & $\begin{array}{c}\text { t-2\%Stn } \\
\text { Data }\end{array}$ & $\begin{array}{c}\text { t-5\%Stn } \\
\text { Data }\end{array}$ & $\mathbf{a}$ \\
\hline $\mathrm{b}$ & 0 & 593 & 345 & $\mathrm{He}$ & 0 & 0 & 0 & $\mathrm{~b}$ \\
\hline $\mathrm{c}$ & 0 & 593 & 414 & $\mathrm{He}$ & 0 & 0 & 0 & $\mathrm{c}$ \\
\hline $\bar{d}$ & 0 & 649 & 345 & $\mathrm{He}$ & 1 & 0 & 0 & $\mathrm{~d}$ \\
\hline e & 0 & 649 & 276 & $\mathrm{He}$ & 0 & 0 & 0 & $\mathrm{e}$ \\
\hline $\mathrm{f}$ & 0 & 649 & 242 & $\mathrm{He}$ & 1 & 0 & 0 & $\mathrm{f}$ \\
\hline $\mathrm{g}$ & 0 & 704 & 276 & $\mathrm{He}$ & 1 & 0 & 0 & $\mathrm{~g}$ \\
\hline $\mathrm{h}$ & 0 & 704 & 207 & $\mathrm{He}$ & 1 & 1 & 1 & $\mathrm{~h}$ \\
\hline $\mathrm{i}$ & 0 & 760 & 138 & $\mathrm{He}$ & 1 & 1 & 1 & $\mathrm{i}$ \\
\hline $\mathrm{j}$ & 0 & 871 & 69 & $\mathrm{He}$ & 1 & 1 & 0 & $\mathrm{j}$ \\
\hline $\mathrm{k}$ & 10,000 & 593 & 414 & $\mathrm{He}$ & 0 & 0 & 0 & $\mathrm{k}$ \\
\hline 1 & 10,000 & 704 & 207 & $\mathrm{He}$ & 1 & 1 & 0 & 1 \\
\hline $\mathrm{m}$ & 20,000 & 704 & 207 & $\mathrm{He}$ & 1 & 1 & 1 & $\mathrm{~m}$ \\
\hline$\downarrow$ & $\downarrow$ & $\downarrow$ & $\downarrow$ & $\downarrow$ & $\downarrow$ & $\downarrow$ & $\downarrow$ & $\downarrow$ \\
\hline
\end{tabular}

t-SampleAge $=$ sample aging time

T-Test $=$ test temperature

TestSts $=$ test stress

$\mathbf{t}-\mathbf{1} \%$ Stn $=$ time for reaching $1 \%$ creep strain

$\mathbf{t}-2 \%$ Stn $=$ time for reaching $2 \%$ creep strain

$\mathbf{t}-\mathbf{5 \%} \mathbf{S t n}=$ time for reaching $5 \%$ creep strain 
Table 10 (cont'd): Number of weld existing creep property data and testing conditions

\begin{tabular}{|c|c|c|c|c|c|c|}
\hline $\mathbf{a}$ & $\begin{array}{c}\text { t-TerStn } \\
\text { Data }\end{array}$ & $\begin{array}{l}\text { t-Rpt } \\
\text { Data }\end{array}$ & $\begin{array}{c}\text { MCR } \\
\text { Data }\end{array}$ & $\begin{array}{c}\text { LdgStn } \\
\text { Data }\end{array}$ & $\begin{array}{c}\text { CpStn } \\
\text { Data }\end{array}$ & $\begin{array}{c}\text { RA } \\
\text { Data }\end{array}$ \\
\hline $\mathrm{b}$ & 0 & 1 & 1 & 1 & 1 & 0 \\
\hline $\mathrm{c}$ & 0 & 2 & 1 & 2 & 2 & 1 \\
\hline $\mathrm{d}$ & 0 & 1 & 1 & 1 & 1 & 1 \\
\hline $\mathrm{e}$ & 0 & 1 & 1 & 1 & 1 & 1 \\
\hline $\mathrm{f}$ & 0 & 1 & 1 & 1 & 1 & 1 \\
\hline $\mathrm{g}$ & 1 & 1 & 1 & 1 & 1 & 1 \\
\hline $\mathrm{h}$ & 1 & 1 & 1 & 1 & 1 & 1 \\
\hline$\overline{\mathrm{i}}$ & 1 & 1 & 1 & 0 & 1 & 1 \\
\hline $\mathrm{j}$ & 0 & 1 & 0 & 1 & 1 & 1 \\
\hline $\mathrm{k}$ & 0 & 1 & 0 & 0 & 1 & 1 \\
\hline 1 & 1 & 1 & 1 & 1 & 1 & 1 \\
\hline $\mathrm{m}$ & 1 & 1 & 1 & 0 & 1 & 1 \\
\hline & $\downarrow$ & $\downarrow$ & $\downarrow$ & $\downarrow$ & $\downarrow$ & $\downarrow$ \\
\hline
\end{tabular}

t-TerStn $=$ time for reaching tertiary creep strain

$\mathbf{t}-\mathbf{R p t}=$ creep rupture time

$\mathbf{M C R}=$ minimum creep rate

LdgStn $=$ loading strain

CpStn $=$ creep rupture strain

$\mathbf{R A}=$ area of reduction 
ASSESSMENT OF EXISTING ALLOY 617 DATA FOR GEN IV NUCLEAR REACTOR APPLICATIONS

Table 11: Number of existing toughness property data and testing conditions

\begin{tabular}{|c|c|c|c|c|c|}
\hline $\begin{array}{c}\text { UnAged } \\
\text { Treat }\end{array}$ & AgeEvn & $\begin{array}{c}\text { T-Age } \\
{ }^{\circ} \mathrm{C} \\
\end{array}$ & $\begin{array}{c}\text { t-Age } \\
\text { h } \\
\end{array}$ & $\begin{array}{c}\text { E-CharpyV } \\
\text { Data } \\
\end{array}$ & DC \\
\hline $\begin{array}{c}\text { solution } \\
\text { annealed }\end{array}$ & N/A & N/A & 0 & 5 & 5 \\
\hline $\mathrm{N} / \mathrm{A}$ & impure He & 871 & 100 & 2 & 5 \\
\hline $\mathrm{N} / \mathrm{A}$ & inert & 871 & 100 & 1 & 5 \\
\hline N/A & inert & 871 & 1000 & 2 & 5 \\
\hline $\mathrm{N} / \mathrm{A}$ & inert & 538 & 10000 & 1 & 5 \\
\hline $\mathrm{N} / \mathrm{A}$ & inert & 704 & 10000 & 1 & 5 \\
\hline $\mathrm{N} / \mathrm{A}$ & inert & 871 & 10000 & 1 & 5 \\
\hline $\mathrm{N} / \mathrm{A}$ & inert & 538 & 20000 & 1 & 5 \\
\hline $\mathrm{N} / \mathrm{A}$ & inert & 704 & 20000 & 1 & 5 \\
\hline N/A & inert & 871 & 20000 & 1 & 5 \\
\hline N/A & inert & 492 & 28300 & 1 & 5 \\
\hline N/A & inert & 538 & 28300 & 1 & 5 \\
\hline$\downarrow$ & $\downarrow$ & $\downarrow$ & $\downarrow$ & $\downarrow$ & $\downarrow$ \\
\hline
\end{tabular}

UnAged Treat $=$ heat treatment for sample without aging

AgeEnv = aging environment for aged sample

T-Age $=$ aging temperature

t-Age $=$ aging time

E-CharpyV = Charpy V impact energy

DC $=$ data classification as discussed in Section 2.1 


\section{MAJOR DATA SOURCES}

Alloy 617 has been selected for many high-temperature and corrosion-resistant applications around the world since its introduction into the marketplace in the early 1970's, and a large amount of data has been generated on the alloy. In this section, the major data sources that have been considered most important to the Gen IV nuclear reactors and processed in the assessment are discussed. Those that have been identified to be of great interest to the Gen IV Materials Handbook but are still under negotiation for access are also introduced. For each data source, basic information germane to the data generation, if available, is provided for a good understanding of the data background.

\subsection{Assessment of Major Data Sources}

\section{ORNL-HTGR Data}

The ORNL-HTGR data source contains the data on Alloy 617 generated at the Oak Ridge National Laboratory (ORNL) for the High-Temperature Gas-Cooled Reactor (HTGR) Project in the 1980's under DOE Contract No. DE-AC05-84OR21400. The source document [McCoy 1985] is a summary report about materials testing results on Alloy 617 and Alloy 618. The report includes tensile properties data generated from 73 tensile tests at temperatures of $24,538,593,649,704,760$, and $871^{\circ} \mathrm{C}(75,1000,1100$, $1200,1300,1400$, and $1600^{\circ} \mathrm{F}$ ), creep properties data generated from 51 creep tests at temperatures of $593,649,704,760$, and $871^{\circ} \mathrm{C}\left(1100,1200,1300,1400\right.$ and $\left.1600^{\circ} \mathrm{F}\right)$, and toughness property data generated from 20 Charpy $\mathrm{V}$ impact tests at room temperature. It also includes the result of 1 tensile test on a specimen after long-term creep testing at $871^{\circ} \mathrm{C}\left(1600^{\circ} \mathrm{F}\right)$. A summary of the tests that generated the ORNLHTGR data is given in Table 12 . The tensile properties include $0.2 \%$ yield strength, ultimate tensile strength, uniform strain, total strain, and reduction in area. The creep properties include times to $1 \%, 2 \%$, and $5 \%$ total strains (Among the three, the time to $1 \%$ total strain is the most important to design.), time to tertiary creep, creep rupture time, creep rupture stress, minimum creep rate, loading strain, creep rupture strain, and reduction in area. The toughness data include Charpy $\mathrm{V}$ impact energy. Based on the Gen IV Materials Handbook Data Classification Criteria, these data are classified as Class 5.

Four heats produced by Huntington Alloy Product Division, predecessor of Special Metals, were used to generate the ORNL-HTGR data. The heat chemical compositions and product forms are presented in Table 13 and Table 14, respectively. Three heats in plate form were used as the base metal; and one heat in wire form was used as the weld filler metal. The welds were produced using the gas tungsten arc (GTA) welding process.

All three base metal heats exhibited fairly coarse grains. The microstructure of Heat XX01A3US exhibited a characteristic of inhomogeneous stringers in the primary working 
direction of the plate. Heat XX09A4UK exhibited a slightly coarser grain size than that in Heat XX01A3US, and its stringers were not nearly as pronounced as those in Heat XX01A3US. The microstructure of the as-received Heat XX14A6UK was not available but that after aging at $593^{\circ} \mathrm{C} \times 10,000$ hours showed no indication of apparent grain size alteration compared to the former two heats. Heat XX14A6UK had the largest grain size of the three but presented no evidence of longitudinal stringers.

Table 12: Summary of ORNL-HTGR data on Alloy 617

\begin{tabular}{|c|c|c|c|c|c|c|c|c|c|}
\hline \multirow{2}{*}{$\begin{array}{c}\text { Test } \\
\text { Type } \\
\end{array}$} & \multirow{2}{*}{$\begin{array}{l}\text { Specimen } \\
\text { Treatment }\end{array}$} & \multirow{2}{*}{$\begin{array}{l}\text { Test } \\
\text { Env }\end{array}$} & \multicolumn{7}{|c|}{ Test Temperature ${ }^{\circ} \mathrm{C}$} \\
\hline & & & $25^{d}$ & 538 & 593 & 649 & 704 & 760 & 871 \\
\hline \multirow{3}{*}{$\begin{array}{c}\text { Tensile } \\
\text { Base } \\
\text { Metal }\end{array}$} & $\begin{array}{c}\mathrm{He} \\
\text { aging }^{\mathrm{a}}\end{array}$ & Air & 7 & & 2 & & 2 & & 2 \\
\hline & $\begin{array}{c}\text { Inert } \\
\text { aging }^{b}\end{array}$ & Air & 11 & 4 & & & 3 & & 3 \\
\hline & $\begin{array}{c}\text { solution } \\
\text { annealing }\end{array}$ & Air & $2^{c}$ & 1 & 1 & 1 & 2 & 1 & 2 \\
\hline \multirow{2}{*}{$\begin{array}{l}\text { Tensile } \\
\text { Weld }^{\mathrm{e}}\end{array}$} & As-welded & Air & 1 & & 1 & 1 & & 1 & 1 \\
\hline & He aging $^{\mathrm{a}}$ & Air & 6 & & 2 & & 2 & & 2 \\
\hline \multirow{5}{*}{$\begin{array}{c}\text { Creep } \\
\text { Base } \\
\text { Metal }\end{array}$} & $\mathrm{He}$ aging $^{\mathrm{a}}$ & $\mathrm{He}$ & & & 3 & & 2 & & 1 \\
\hline & inert & Air & & & & 2 & & & \\
\hline & aging $^{b}$ & $\mathrm{He}$ & & & & & 1 & 1 & 1 \\
\hline & solution & Air & & & 2 & 1 & 1 & 2 & 4 \\
\hline & annealing & $\mathrm{He}$ & & & 1 & 4 & 3 & 3 & 3 \\
\hline \multirow{2}{*}{$\begin{array}{l}\text { Creep } \\
\text { Weld }^{\mathrm{e}}\end{array}$} & As-welded & $\mathrm{He}$ & & & 3 & 3 & 2 & 1 & 1 \\
\hline & He aging $^{a}$ & $\mathrm{He}$ & & & 1 & & 2 & & \\
\hline \multirow{3}{*}{$\begin{array}{c}\text { Charpy } \\
\text { Base } \\
\text { Metal }\end{array}$} & 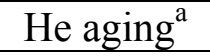 & Air & 2 & & & & & & 1 \\
\hline & $\begin{array}{c}\text { inert } \\
\text { aging }^{b}\end{array}$ & Air & 13 & & & & & & \\
\hline & $\begin{array}{c}\text { solution } \\
\text { annealing }\end{array}$ & Air & 5 & & & & & & \\
\hline
\end{tabular}

a. Specimens were aged in simulated HTGR helium mostly for 10000 or 20000 hours at the same temperature as the test temperature, except room temperature.

b. Block material was aged in steam; then the specimens were machined.

c. One of the two was reported with unknown sample treatment, solution annealing assumed.

d. Test temperatures of 22,24 and $25^{\circ} \mathrm{C}$ were reported, all are summarized as room temperature $=25^{\circ} \mathrm{C}$.

e. Weld represents weld metal or weldment.

The data for welds were generated from three types of specimens: base metal, weld metal, and weldment. The base metal specimens were machined with their axis parallel to the primary working direction of the original plate. The weld metal specimens were cut parallel to the weld direction with the gage section composed totally of the weld 
metal. And the weldment specimens were produced with the gage section spanning perpendicular to the weld and containing the base metal, the weld metal, and the heat affected zone.

Table 13: Compositions (wt. \%) of the Alloy 617 heats used for the ORNL-HTGR data generation [McCoy285T1]

\begin{tabular}{|c|c|c|c|c|c|c|c|c|c|c|}
\hline Heat & Ni & Cr & Co & Mn & Mo & Fe & Al & Si & C & S \\
\hline XX01A3US & 57.35 & 20.30 & 11.72 & 0.05 & 8.58 & 1.01 & 0.76 & 0.16 & 0.07 & 0.004 \\
\hline XX09A4UK & 55.11 & 21.83 & 12.55 & 0.02 & 8.79 & 0.38 & 1.15 & 0.10 & 0.07 & 0.001 \\
\hline XX14A6UK & 55.13 & 21.74 & 12.32 & 0.02 & 8.91 & 0.53 & 1.11 & 0.18 & 0.06 & 0.002 \\
\hline XX09A9UK & 54.38 & 22.14 & 12.66 & 0.02 & 8.79 & 0.39 & 1.37 & 0.17 & 0.08 & 0.001 \\
\hline
\end{tabular}

Table 14: Product characterization of the Alloy 617 heats used for the ORNL-HTGR data generation [McCoy85T2]

\begin{tabular}{|c|c|c|c|}
\hline Heat & Product Form & Grain Size $(\mu \mathbf{m})$ & Heat Treatment \\
\hline XX01A3US & Plate, $13 \mathrm{~mm}(0.5$ in.) thick & 130 & $\begin{array}{c}\text { Solution annealed } \\
\text { by vendor }\end{array}$ \\
\hline XX09A4UK & Plate, $9.5 \mathrm{~mm}(0.38$ in. $)$ thick & 210 & Unknown \\
\hline XX14A6UK & Plate, $13 \mathrm{~mm}(0.5$ in.) thick & 270 & $\begin{array}{c}\text { Solution annealed } \\
\text { by vendor }\end{array}$ \\
\hline XX09A9UK & Wire, $1.1 \mathrm{~mm}(0.045$ in. $)$ diameter & & Unknown \\
\hline
\end{tabular}

The tensile properties data were generated from the material aged at temperatures of $482,538,593,704$, and $871^{\circ} \mathrm{C}\left(900,1000,1100,1300\right.$, and $\left.1600^{\circ} \mathrm{F}\right)$ for 2500,10000 , 20000,26117 and 28300 hours. The creep properties data were generated from materials aged at $482,538,593,704$, and $871^{\circ} \mathrm{C}\left(900,1000,1100,1300\right.$, and $\left.1600^{\circ} \mathrm{F}\right)$ for 10000 , 20000 and 28300 hours. And the toughness data were generated from material aged at $492,538,704,871,\left(918,1000,1300,1600^{\circ} \mathrm{F}\right)$ for $100,1000,10000,20000$ and 28300 hours. [Note: Although the original report had $492^{\circ} \mathrm{C}\left(918^{\circ} \mathrm{F}\right)$ as the toughness material aging temperature, from the context the $492^{\circ} \mathrm{C}\left(918^{\circ} \mathrm{F}\right)$ might be a typo for $482^{\circ} \mathrm{C}$ $\left(900^{\circ} \mathrm{F}\right)$. Verification is underway.] The material was aged in two environments, i. e., inert and simulated HTGR helium environments. The inert environment included two situations: In the first situation, the specimens were stacked in metal containers, which were then evacuated, filled with an inert gas, and welded shut for aging. In the second situation, the material was exposed to a steam loop in the form of blocks, and specimens were machined out of the blocks after the exposure, so the only important effect from the exposure was the thermal aging because any effects from chemical interaction with the steam environment were machined away. The HTGR helium-aged specimens were aged continuously in specially designed stainless steel retorts, except that the retort operating at $871^{\circ} \mathrm{C}\left(1600^{\circ} \mathrm{F}\right)$ was replaced with an aluminum oxide retort in the middle of the aging process and the specimens were simply stacked in place. 
The simulated HTGR helium for generating the ORNL-HTGR creep data was premixed and flowed into the testing environmental chamber at rates of 15 to 100 $\mathrm{cm}^{3} / \mathrm{min}$. The helium composition in the test chamber was 34.0 (337) $\mathrm{H}_{2}, 3.2$ (32) $\mathrm{CH}_{4}$, 1.9 (19) $\mathrm{CO}, 0.2(2) \mathrm{H}_{2} \mathrm{O}$, and $<0.05(<0.5) \mathrm{N}_{2}$ in pascals (micro atmospheres). Oxygen was removed by reaction with the $\mathrm{H}_{2}$ as the gas passed through a furnace at $500^{\circ} \mathrm{C}$ $\left(932^{\circ} \mathrm{F}\right)$.

The standard test startup for generating the ORNL-HTGR creep data consisted of assembling the new test specimen in the chamber, evacuating air from the chamber, pressurizing the chamber with test gas and establishing flow at $83 \mathrm{kPa}$ gage (12 psig), leak-checking with a helium sniffer, heating to $400^{\circ} \mathrm{C}\left(752^{\circ} \mathrm{F}\right)$ and holding for at least 24 hours until moisture was less than $10 \mathrm{ppm}$, heating to test temperature, and applying load to start the test. All impurities were initially high, but the outgassing period at $400^{\circ} \mathrm{C}$ $\left(752^{\circ} \mathrm{F}\right)$ allowed the gas composition to reach the desired operating level. Some elevation in impurity levels (less than a factor of 2) occurred during heating to the test temperature, but the gas reached the desired operating levels within 24 hours. 
ASSESSMENT OF EXISTING ALLOY 617 DATA

FOR GEN IV NUCLEAR REACTOR APPLICATIONS

ORIGINAL ORNL - HTGR DATA 


\begin{tabular}{l|l|l|l|}
\hline Data ID: & McCoy285T3 & Data Class: & 5 \\
\hline Project: & ORNL US DOE HTGR Project & Reference: & McCoy 1985 \\
\hline $\begin{array}{l}\text { Comment: Material was aged in static air in the form of blocks before specimens were } \\
\text { machined. The aging environment is therefore considered inert. }\end{array}$
\end{tabular}

Table 3. Tensile properties of Inconel 617 (heat XX01A3US) as received and after aging in static air

[Specimens machined after aging. Gage sections were $31.8 \mathrm{~mm}$ (1.25 in.) long by $6.4 \mathrm{~mm}$

$(0.25 \mathrm{in.)}$ in diameter.]

\begin{tabular}{|c|c|c|c|c|c|c|c|c|c|c|}
\hline \multirow{3}{*}{ Test } & \multicolumn{3}{|c|}{ Aging condition } & \multirow{2}{*}{\multicolumn{2}{|c|}{$\begin{array}{l}0.2 \% \text { Yield } \\
\text { strength }\end{array}$}} & \multirow{2}{*}{\multicolumn{2}{|c|}{$\begin{array}{l}\text { Ultimate } \\
\text { tensile } \\
\text { strength }\end{array}$}} & \multirow{2}{*}{\multicolumn{2}{|c|}{ Elongation ${ }^{a}(\%)$}} & \multirow{3}{*}{$\begin{array}{c}\text { Reduc- } \\
\text { tion } \\
\text { of } \\
\text { area } \\
(\%)\end{array}$} \\
\hline & \multicolumn{2}{|c|}{ Temperature } & \multirow{2}{*}{$\begin{array}{l}\text { Time } \\
\text { (h) }\end{array}$} & & & & & & & \\
\hline & $\left({ }^{\circ} \mathrm{C}\right)$ & $\left({ }^{\circ} \mathrm{F}\right)$ & & $(\mathrm{MPa})$ & (ksi) & $(\mathrm{MPa})$ & (ks1) & Uniform & Total & \\
\hline \multicolumn{11}{|c|}{ Tested at $22^{\circ} \mathrm{C}\left(72^{\circ} \mathrm{P}\right)$} \\
\hline $\begin{array}{l}16545 \\
16546\end{array}$ & & & $\begin{array}{l}0 \\
0\end{array}$ & $\begin{array}{l}303 \\
301\end{array}$ & $\begin{array}{l}43.9 \\
43.7\end{array}$ & $\begin{array}{l}747 \\
757\end{array}$ & $\begin{array}{l}108.3 \\
109.8\end{array}$ & $\begin{array}{l}54.1 \\
59.6\end{array}$ & $\begin{array}{l}54.3 \\
60.9\end{array}$ & $\begin{array}{l}36.2 \\
40.7\end{array}$ \\
\hline 16547 & & & 0 & 305 & 44.3 & 754 & 109.3 & 57.4 & 57.9 & 32.4 \\
\hline 17897 & 538 & 1000 & 2,500 & 358 & 51.9 & 794 & 115.2 & 66.0 & 68.1 & 49.3 \\
\hline 17898 & 583 & 1000 & 2,500 & 360 & 52.2 & 796 & 115.5 & 67.6 & 69.8 & 48.6 \\
\hline 17901 & 704 & 1300 & 2,500 & 371 & 53.8 & 847 & 122.9 & 39.2 & 39.2 & 32.0 \\
\hline 17902 & 704 & 1300 & 2,500 & 372 & 54.7 & 848 & 123.1 & 44.1 & 44.1 & 35.0 \\
\hline $\begin{array}{l}17905^{b} \\
17906\end{array}$ & $\begin{array}{l}871 \\
871\end{array}$ & $\begin{array}{l}1600 \\
1600\end{array}$ & $\begin{array}{l}2,500 \\
2,500\end{array}$ & $\begin{array}{l}331 \\
334\end{array}$ & $\begin{array}{l}48.0 \\
48.5\end{array}$ & $\begin{array}{l}797 \\
822\end{array}$ & $\begin{array}{l}115.6 \\
119.2\end{array}$ & $\begin{array}{l}29.9 \\
34.6\end{array}$ & $\begin{array}{l}29.9 \\
34.6\end{array}$ & $\begin{array}{l}25.0 \\
29.3\end{array}$ \\
\hline IC43 & 538 & 1000 & 10,000 & 392 & 56.9 & 805 & 116.8 & 53.3 & 53.4 & 43.8 \\
\hline IC25 & 704 & 1300 & 10,000 & 369 & 53.5 & 798 & 115.7 & 29.6 & 29.6 & 23.3 \\
\hline IC7 & 871 & 1600 & 10,000 & 290 & 42.0 & 695 & 100.8 & 28.3 & 28.3 & 21.0 \\
\hline IC38 & 538 & 1000 & 20,000 & 379 & 55.0 & 822 & 119.2 & 62.4 & 62.7 & 43.7 \\
\hline IC2O & 704 & 1300 & 20,000 & 395 & 57.3 & 813 & 117.9 & 21.1 & 21.1 & 17.3 \\
\hline ICO2 & 871 & 1600 & 20,000 & 300 & 43.5 & 658 & 95.4 & 20.2 & 20.2 & 15.9 \\
\hline \multicolumn{11}{|c|}{ Tested at $538^{\circ} \mathrm{C}\left(1000^{\circ} \mathrm{F}\right)$} \\
\hline $\begin{array}{l}16657 \\
16658\end{array}$ & & & $\begin{array}{l}0 \\
0\end{array}$ & $\begin{array}{l}216 \\
207\end{array}$ & $\begin{array}{l}31.3 \\
30.0\end{array}$ & $\begin{array}{l}610 \\
606\end{array}$ & $\begin{array}{l}88.5 \\
87.9\end{array}$ & $\begin{array}{l}64.7 \\
61.1\end{array}$ & $\begin{array}{l}67.3 \\
64.4\end{array}$ & $\begin{array}{l}49.8 \\
46.1\end{array}$ \\
\hline 17899 & 538 & 1000 & 2,500 & 260 & 37.7 & 638 & 92.5 & 63.4 & 68.5 & 51.7 \\
\hline 17900 & 538 & 1000 & 2,500 & 259 & 37.6 & 638 & 92.5 & 66.3 & 69.2 & 48.9 \\
\hline IC44 & 538 & 1000 & 10,000 & 222 & 32.2 & 669 & 97.0 & 36.4 & 36.5 & 30.5 \\
\hline IC26 & 704 & 1300 & 10,000 & 274 & 39.8 & 629 & 91.3 & 58.9 & 58.9 & 46.3 \\
\hline IC39 & 538 & 1000 & 20,000 & 281 & 40.8 & 621 & 90.0 & 64.6 & 66.2 & 51.4 \\
\hline \multicolumn{11}{|c|}{ Tested at $704^{\circ} \mathrm{C}\left(1300^{\circ} \mathrm{F}\right)$} \\
\hline $\begin{array}{l}16664 \\
16665\end{array}$ & & & $\begin{array}{l}0 \\
0\end{array}$ & $\begin{array}{l}199 \\
196\end{array}$ & $\begin{array}{l}28.8 \\
28.4\end{array}$ & $\begin{array}{l}466 \\
443\end{array}$ & $\begin{array}{l}67.6 \\
64.2\end{array}$ & $\begin{array}{l}29.3 \\
25.9\end{array}$ & $\begin{array}{l}68.0 \\
69.9\end{array}$ & $\begin{array}{l}49.3 \\
53.1\end{array}$ \\
\hline 16666 & & & 0 & 196 & 28.4 & 454 & 65.9 & 29.9 & 74.7 & 52.5 \\
\hline 17903 & 704 & 1300 & 2,500 & 257 & 37.3 & 502 & 72.8 & 16.2 & 75.3 & 57.6 \\
\hline $17904^{c}$ & 704 & 1300 & 2,500 & 256 & 37.1 & 475 & 69.0 & 14.4 & & \\
\hline IC27 & 704 & 1300 & 10,000 & 211 & 30.6 & 461 & 66.9 & 16.9 & 68.8 & 56.3 \\
\hline IC21 & 704 & 1300 & 20,000 & 285 & 41.3 & 525 & 76.2 & 13.6 & 53.1 & 48.5 \\
\hline \multicolumn{11}{|c|}{ Tested at $871^{\circ} \mathrm{C}\left(1600^{\circ} \mathrm{F}\right)$} \\
\hline $\begin{array}{l}16672 \\
16673\end{array}$ & & & $\begin{array}{l}0 \\
0\end{array}$ & $\begin{array}{l}181 \\
189\end{array}$ & $\begin{array}{l}26.3 \\
27.4\end{array}$ & $\begin{array}{l}194 \\
194\end{array}$ & $\begin{array}{l}28.1 \\
28.1\end{array}$ & $\begin{array}{l}3.2 \\
3.2\end{array}$ & $\begin{array}{l}88.0 \\
91.6\end{array}$ & $\begin{array}{l}87.8 \\
86.0\end{array}$ \\
\hline 17907 & 871 & 1600 & 2,500 & 154 & 22.3 & 168 & 24.3 & 5.1 & & \\
\hline 17908 & 871 & 1600 & 2,500 & 170 & 24.6 & 179 & 25.9 & 4.3 & 98.2 & 91.0 \\
\hline IC8 & 871 & 1600 & 10,000 & 170 & 24.7 & 181 & 26.2 & 8.0 & 90.9 & 79.2 \\
\hline IC3 & 871 & 1600 & 20,000 & 180 & 26.1 & 255 & 37.0 & 8.8 & 89.9 & 72.8 \\
\hline
\end{tabular}

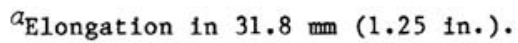

$b_{\text {Broke at gage mark. }}$

$c_{\text {Pull rod broke before specimen broke. }}$ 


\begin{tabular}{|l|l|l|l|}
\hline Data ID: & McCoy285T4 & Data Class: & 5 \\
\hline Project: & ORNL US DOE HTGR Project & Reference: & McCoy 1985 \\
\hline Comment: & \multicolumn{2}{|l}{} \\
\hline
\end{tabular}

Table 4. Tensile properties of Inconel 617 (heat XX01A3US) after aging in HTGR-He

[Specimens machined before aging. Gage sections were $25.4 \mathrm{~mm}(1.00 \mathrm{in.})$ long by $3.18 \mathrm{~mm}(0.125 \mathrm{in.})$ in diameter.]

\begin{tabular}{|c|c|c|c|c|c|c|c|c|c|c|}
\hline \multirow{3}{*}{ Spec1men } & \multicolumn{3}{|c|}{ Aging condition } & \multirow{2}{*}{\multicolumn{2}{|c|}{$\begin{array}{l}0.2 \% \text { Yield } \\
\text { strength }\end{array}$}} & \multirow{2}{*}{\multicolumn{2}{|c|}{$\begin{array}{l}\text { Ultimate } \\
\text { tensile } \\
\text { strength }\end{array}$}} & \multicolumn{2}{|c|}{ Elongation (z) } & \multirow{3}{*}{$\begin{array}{c}\text { Reduc- } \\
\text { t1on } \\
\text { of } \\
\text { area } \\
(z)\end{array}$} \\
\hline & \multicolumn{2}{|c|}{ Temperature } & \multirow{2}{*}{$\begin{array}{l}\text { Time } \\
\text { (h) }\end{array}$} & & & & & \multirow{2}{*}{ Uniform } & \multirow{2}{*}{ Total } & \\
\hline & $\left({ }^{\circ} \mathrm{C}\right)$ & $\left({ }^{\circ} \mathrm{F}\right)$ & & $(\mathrm{MPa})$ & (ks1) & (MPa) & (k81) & & & \\
\hline \multicolumn{11}{|c|}{ Tested at $24^{\circ} \mathrm{C}\left(75^{\circ} \mathrm{P}\right)$} \\
\hline $\begin{array}{l}I-100 \\
I-113 \\
I-104 \\
I-117 \\
I-108 \\
I-121\end{array}$ & $\begin{array}{l}593 \\
593 \\
704 \\
704 \\
871 \\
871\end{array}$ & $\begin{array}{l}1100 \\
1100 \\
1300 \\
1300 \\
1600 \\
1600\end{array}$ & $\begin{array}{l}10,000 \\
20,000 \\
10,000 \\
20,000 \\
10,000 \\
20,000\end{array}$ & $\begin{array}{l}316 \\
510 \\
531 \\
523 \\
392 \\
303\end{array}$ & $\begin{array}{l}45.9 \\
74.0 \\
77.0 \\
75.8 \\
56.8 \\
44.0\end{array}$ & $\begin{array}{l}737 \\
919 \\
818 \\
794 \\
623 \\
509\end{array}$ & $\begin{array}{r}106.9 \\
133.3 \\
118.6 \\
115.3 \\
90.3 \\
73.8\end{array}$ & $\begin{array}{r}53.5 \\
24.4 \\
14.1 \\
9.6 \\
11.4 \\
6.0\end{array}$ & $\begin{array}{r}59.6 \\
24.8 \\
14.3 \\
10.0 \\
11.4 \\
6.2\end{array}$ & $\begin{array}{r}57.1 \\
29.1 \\
14.6 \\
15.5 \\
14.2 \\
6.8\end{array}$ \\
\hline \multicolumn{11}{|c|}{ Tested at $593^{\circ} \mathrm{C}\left(1100^{\circ} \mathrm{F}\right)$} \\
\hline $\begin{array}{l}I-101 \\
I-112\end{array}$ & $\begin{array}{l}593 \\
593\end{array}$ & $\begin{array}{l}1100 \\
1100\end{array}$ & $\begin{array}{l}10,000 \\
20,000\end{array}$ & $\begin{array}{l}238 \\
378\end{array}$ & $\begin{array}{l}34.5 \\
54.8\end{array}$ & $\begin{array}{l}572 \\
687\end{array}$ & $\begin{array}{l}82.9 \\
99.6\end{array}$ & $\begin{array}{l}44.6 \\
24.7\end{array}$ & $\begin{array}{l}48.2 \\
25.6\end{array}$ & $\begin{array}{l}44.4 \\
30.6\end{array}$ \\
\hline \multicolumn{11}{|c|}{ Tested at $704^{\circ} \mathrm{C}\left(1300^{\circ} \mathrm{F}\right)$} \\
\hline $\begin{array}{l}I-105 \\
I-116\end{array}$ & $\begin{array}{l}704 \\
704\end{array}$ & $\begin{array}{l}1300 \\
1300\end{array}$ & $\begin{array}{l}10,000 \\
20,000\end{array}$ & $\begin{array}{l}387 \\
378\end{array}$ & $\begin{array}{l}56.1 \\
54.8\end{array}$ & $\begin{array}{l}706 \\
698\end{array}$ & $\begin{array}{l}102.4 \\
101.2\end{array}$ & $\begin{array}{l}22.0 \\
17.5\end{array}$ & $\begin{array}{l}25.2 \\
19.3\end{array}$ & $\begin{array}{l}26.6 \\
22.0\end{array}$ \\
\hline \multicolumn{11}{|c|}{ Tested at $871^{\circ} \mathrm{C}\left(1600^{\circ} \mathrm{F}\right)$} \\
\hline $\begin{array}{r}I-109 \\
I-120 \\
\end{array}$ & $\begin{array}{l}871 \\
871\end{array}$ & $\begin{array}{l}1600 \\
1600\end{array}$ & $\begin{array}{l}10,000 \\
20,000\end{array}$ & $\begin{array}{l}219 \\
171\end{array}$ & $\begin{array}{l}31.7 \\
24.8\end{array}$ & $\begin{array}{l}283 \\
280\end{array}$ & $\begin{array}{l}41.0 \\
40.6\end{array}$ & $\begin{array}{l}8.0 \\
5.4\end{array}$ & $\begin{array}{l}59.4 \\
63.4\end{array}$ & $\begin{array}{l}61.2 \\
54.4\end{array}$ \\
\hline
\end{tabular}

\begin{tabular}{|l|l|l|l|}
\hline Data ID: & McCoy285T5 & Data Class: & 5 \\
\hline Project: & ORNL US DOE HTGR Project & Reference: & McCoy 1985 \\
\hline Comment: & \multicolumn{2}{l|}{} \\
\hline
\end{tabular}

Table 5. Tensile properties of as-recelved Inconel 617 (heat XX09A4UK)

\begin{tabular}{|c|c|c|c|c|c|c|c|c|c|}
\hline \multirow{2}{*}{ Specimen } & \multicolumn{2}{|c|}{$\begin{array}{c}\text { Test } \\
\text { temperature }\end{array}$} & \multicolumn{2}{|c|}{$\begin{array}{l}0.2 \% \text { Yield } \\
\text { strength }\end{array}$} & \multicolumn{2}{|c|}{$\begin{array}{l}\text { Ultimate } \\
\text { tensile } \\
\text { strength }\end{array}$} & \multicolumn{2}{|c|}{ Elongation (\%) } & \multirow{2}{*}{$\begin{array}{c}\text { Reduc- } \\
\text { tion } \\
\text { of } \\
\text { area } \\
(\%)\end{array}$} \\
\hline & $\left({ }^{\circ} \mathrm{C}\right)$ & $\left({ }^{\circ} \mathrm{F}\right)$ & $(\mathrm{MPa})$ & (ksi) & (MPa) & (ks1) & Uniform & Total & \\
\hline 24 & 25 & 77 & 394 & 57.1 & 766 & 111 & 53.7 & 56.5 & 62.9 \\
\hline 200 & 25 & 77 & 363 & 52.7 & 765 & 111 & 48.7 & 50.2 & 53.5 \\
\hline 201 & 593 & 1100 & 216 & 31.4 & 556 & 80.7 & 49.5 & 52.2 & 48.6 \\
\hline 202 & 649 & 1200 & 235 & 34.2 & 549 & 79.7 & 46.0 & 48.3 & 45.4 \\
\hline 203 & 704 & 1300 & 290 & 42.1 & 502 & 72.8 & 32.0 & 34.0 & 39.8 \\
\hline 204 & 760 & 1400 & 331 & 48.1 & 556 & 80.7 & 15.4 & 19.9 & 17.4 \\
\hline 205 & 871 & 1600 & 268 & 38.8 & 284 & 41.2 & 13.0 & 20.4 & 26.1 \\
\hline
\end{tabular}




\begin{tabular}{|l|l|l|l|}
\hline Data ID: & McCoy285T7 & Data Class: & 5 \\
\hline Project: & ORNL US DOE HTGR Project & Reference: & McCoy 1985 \\
\hline Comment:
\end{tabular}

Table 7. Tensile properties of Inconel 617 (heat XX01A3US) base metal at $75^{\circ} \mathrm{C}$ after aging $28,300 \mathrm{~h}$ in steam

(Sample machined from block after exposure, so aging environment was inert)

\begin{tabular}{|c|c|c|c|c|c|c|c|c|}
\hline \multicolumn{2}{|c|}{$\begin{array}{c}\text { Aging } \\
\text { temperature }\end{array}$} & \multicolumn{2}{|c|}{$\begin{array}{l}0.2 \% \text { Yield } \\
\text { strength }\end{array}$} & \multicolumn{2}{|c|}{$\begin{array}{l}\text { Ultimate } \\
\text { tensile } \\
\text { strength }\end{array}$} & \multicolumn{2}{|c|}{ Elongation $(\%)$} & \multirow{2}{*}{$\begin{array}{c}\text { Reduc- } \\
\text { tion } \\
\text { of } \\
\text { area } \\
(\%)\end{array}$} \\
\hline$\left({ }^{\circ} \mathrm{C}\right)$ & $\left({ }^{\circ} \mathrm{F}\right)$ & (MPa) & (ksi) & $(\mathrm{MPa})$ & (ksi) & Uniform & Total & \\
\hline 482 & 900 & 401 & 58.2 & 859 & 124.6 & 52.2 & 52.8 & 48.9 \\
\hline 538 & 1000 & 387 & 56.1 & 838 & 121.5 & 51.8 & 52.5 & 46.6 \\
\hline
\end{tabular}

\begin{tabular}{|l|l|l|l|}
\hline Data ID: & McCoy285T8 & Data Class: & 5 \\
\hline Project: & ORNL US DOE HTGR Project & Reference: & McCoy 1985 \\
\hline Comment: & \multicolumn{2}{|l|}{} \\
\hline
\end{tabular}

Table 8. Properties of Inconel 617 weld metal as welded

[Base material heat XX14A6UK, filler wire XX09A9UK, specimen gage section $25 \mathrm{~mm}$ (1.0 in.) long by

$3.2 \mathrm{~mm}(0.13 \mathrm{in.})$ in diameter, tested at strain rate of $0.05 / \mathrm{min}$ ]

\begin{tabular}{|c|c|c|c|c|c|c|c|c|}
\hline \multicolumn{2}{|c|}{$\begin{array}{c}\text { Test } \\
\text { temperature }\end{array}$} & \multicolumn{2}{|c|}{$\begin{array}{l}0.2 \% \text { Yield } \\
\text { strength }\end{array}$} & \multicolumn{2}{|c|}{$\begin{array}{l}\text { U1timate } \\
\text { tensile } \\
\text { strength }\end{array}$} & \multicolumn{2}{|c|}{ Elongation (\%) } & \multirow{2}{*}{$\begin{array}{c}\text { Reduc- } \\
\text { tion } \\
\text { of } \\
\text { area } \\
(\%)\end{array}$} \\
\hline$\left({ }^{\circ} \mathrm{C}\right)$ & $\left({ }^{\circ} \mathrm{F}\right)$ & (MPa) & (ks1) & (MPa) & (ksi) & Un1form & Tota1 & \\
\hline 25 & 77 & 537 & 77.9 & 813 & 117.9 & 32.4 & 35.6 & 54.6 \\
\hline 593 & 1100 & 393 & 57.0 & 601 & 87.1 & 31.3 & 32.2 & 45.6 \\
\hline 649 & 1200 & 367 & 53.2 & 567 & 82.3 & 35.7 & 36.8 & 38.4 \\
\hline 760 & 1400 & 412 & 59.8 & 547 & 79.4 & 10.3 & 25.7 & 28.8 \\
\hline 871 & 1600 & 272 & 39.4 & 288 & 41.8 & 6.0 & 42.8 & 71.1 \\
\hline
\end{tabular}




\begin{tabular}{|l|l|l|l|}
\hline Data ID: & McCoy285T9 & Data Class: & 5 \\
\hline Project: & ORNL US DOE HTGR Project & Reference: & McCoy 1985 \\
\hline Comment:
\end{tabular}

Table 9. Tensile properties of Inconel 617 transverse weld specimens after aging in HTGR-He

(Welds made by the gas tungsten arc process from heat XX14A6UK base metal and XX09A9UK filler wire)

\begin{tabular}{|c|c|c|c|c|c|c|c|c|c|c|c|}
\hline \multirow{3}{*}{ Specimen } & \multicolumn{3}{|c|}{ Aging condition } & \multirow{2}{*}{\multicolumn{2}{|c|}{$\begin{array}{l}0.27 \text { Yield } \\
\text { strength }\end{array}$}} & \multirow{2}{*}{\multicolumn{2}{|c|}{$\begin{array}{l}\text { U1timate } \\
\text { tensile } \\
\text { strength }\end{array}$}} & \multirow{2}{*}{\multicolumn{2}{|c|}{ Elongation (z) }} & \multirow{3}{*}{$\begin{array}{c}\text { Reduc- } \\
\text { tion } \\
\text { of } \\
\text { area } \\
(z)\end{array}$} & \multirow{3}{*}{$\begin{array}{r}\text { Fa1lure } \\
\text { location }\end{array}$} \\
\hline & \multicolumn{2}{|c|}{ Temperature } & \multirow{2}{*}{$\begin{array}{l}\text { Time } \\
\text { (h) }\end{array}$} & & & & & & & & \\
\hline & $\left({ }^{\circ} \mathrm{C}\right)$ & $\left({ }^{\circ} \mathrm{F}\right)$ & & (MPa) & (ks1) & $(\mathrm{MPa})$ & (ksi) & 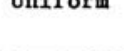 & Total & & \\
\hline \multicolumn{12}{|c|}{ Tested at $24^{\circ} \mathrm{C}\left(75^{\circ} \mathrm{F}\right)$} \\
\hline$I-148$ & 593 & 1100 & 10,000 & 330 & 47.8 & 1004 & 145.6 & 26.0 & 26.3 & 28.3 & Base \\
\hline I-161 & 593 & 1100 & 20,000 & 676 & 98.0 & 1020 & 148.0 & 19.5 & 19.9 & 10.6 & Base \\
\hline I-152 & 704 & 1300 & 10,000 & 331 & 48.0 & 927 & 134.4 & 14.2 & 14.4 & 21.0 & Base \\
\hline I-165 & 704 & 1300 & 20,000 & 626 & 90.3 & 867 & 125.8 & 5.0 & 5.2 & 8.3 & Base \\
\hline I-156 & 871 & 1600 & 10,000 & 394 & 57.1 & 450 & 65.2 & 0.7 & 0.7 & 3.2 & Base \\
\hline $\mathrm{I}-169$ & 871 & 1600 & 20,000 & 356 & 51.6 & 476 & 69.0 & 0.8 & 0.8 & 3.3 & Base \\
\hline \multicolumn{12}{|c|}{ Tested at $593^{\circ} \mathrm{C}\left(1100^{\circ} \mathrm{F}\right)$} \\
\hline $\begin{array}{l}I-168 \\
I-160\end{array}$ & $\begin{array}{l}593 \\
593\end{array}$ & $\begin{array}{l}1100 \\
1100\end{array}$ & $\begin{array}{l}10,000 \\
20,000\end{array}$ & $\begin{array}{l}259 \\
623\end{array}$ & $\begin{array}{l}37.6 \\
90.4\end{array}$ & $\begin{array}{l}514 \\
800\end{array}$ & $\begin{array}{r}74.6 \\
116.0\end{array}$ & $\begin{array}{l}29.0 \\
15.2\end{array}$ & $\begin{array}{l}29.4 \\
16.2\end{array}$ & $\begin{array}{l}33.3 \\
26.6\end{array}$ & $\begin{array}{l}\text { Weld } \\
\text { Base }\end{array}$ \\
\hline \multicolumn{12}{|c|}{ Tested at $704^{\circ} \mathrm{C}\left(1300^{\circ} \mathrm{F}\right)$} \\
\hline $\begin{array}{l}I-153 \\
I-164\end{array}$ & $\begin{array}{l}704 \\
704\end{array}$ & $\begin{array}{l}1300 \\
1300\end{array}$ & $\begin{array}{l}10,000 \\
20,000\end{array}$ & $\begin{array}{l}460 \\
445\end{array}$ & $\begin{array}{l}66.7 \\
64.5\end{array}$ & $\begin{array}{l}756 \\
740\end{array}$ & $\begin{array}{l}109.7 \\
107.4\end{array}$ & $\begin{array}{l}15.7 \\
12.2\end{array}$ & $\begin{array}{l}19.9 \\
15.6\end{array}$ & $\begin{array}{r}35.0 \\
8.5\end{array}$ & $\begin{array}{l}\text { Weld } \\
\text { Base }\end{array}$ \\
\hline \multicolumn{12}{|c|}{ Tested at $871^{\circ} \mathrm{C}\left(1600^{\circ} \mathrm{F}\right)$} \\
\hline $\begin{array}{l}I-157 \\
I-168\end{array}$ & 871 & 1600 & 10,000 & 223 & 32.4 & 270 & 39.1 & 6.0 & 26.8 & 56.0 & Weld \\
\hline & 871 & 1600 & 20,000 & 218 & 31.6 & 277 & 40.2 & 2.3 & 23.9 & 71.8 & We1d \\
\hline
\end{tabular}

\begin{tabular}{|l|l|l|l|}
\hline Data ID: & McCoy285T18 & Data Class: & 5 \\
\hline Project: & ORNL US DOE HTGR Project & Reference: & McCoy 1985 \\
\hline Comment:
\end{tabular}

Table 18. Tensile properties at $25^{\circ} \mathrm{C}$ of Inconel 617 and 618 after exposures in creep tests

\begin{tabular}{|c|c|c|c|c|c|c|c|c|}
\hline \multirow{2}{*}{ Material } & \multirow{2}{*}{ Pretest condition } & \multicolumn{2}{|c|}{$\begin{array}{l}0.2 \% \text { Yield } \\
\text { stress }\end{array}$} & \multicolumn{2}{|c|}{$\begin{array}{l}\text { Ultimate } \\
\text { tensile stress }\end{array}$} & \multicolumn{2}{|c|}{ Elongat1on (\%) } & \multirow{2}{*}{$\begin{array}{l}\text { Reduc- } \\
\text { tion } \\
\text { of area } \\
(\%)\end{array}$} \\
\hline & & (MPa) & (ksi) & (MPa) & (ks1) & Uniform & Total & \\
\hline $\begin{array}{l}\text { Incone1 } 617 \\
\quad \mathrm{XX09A} 4 \mathrm{UK}\end{array}$ & $\begin{array}{l}\text { Test } 21602,871^{\circ} \mathrm{C}, 21 \mathrm{MPa}, \\
26,117 \mathrm{~h}, 0.233 \% \mathrm{C}\end{array}$ & 326 & 47.3 & 446 & 64.7 & 4.0 & 4.0 & 0.3 \\
\hline $\begin{array}{c}\text { Inconel } 618, \\
\text { transverse } \\
\text { weld, Y09B9 }\end{array}$ & $\begin{array}{l}\text { Test } 23083,760^{\circ} \mathrm{C}, 69 \mathrm{MPa} \text {, } \\
11,158 \mathrm{~h}, 0.062 \% \mathrm{C}\end{array}$ & 565 & 53.0 & 582 & 84.4 & 19.7 & 19.7 & 22.3 \\
\hline
\end{tabular}


ASSESSMENT OF EXISTING ALLOY 617 DATA FOR GEN IV NUCLEAR REACTOR APPLICATIONS

\begin{tabular}{|l|l|l|l|}
\hline Data ID: & McCoy285T13 & Data Class: & 5 \\
\hline Project: & ORNL US DOE HTGR Project & Reference: & McCoy 1985 \\
\hline Comment:
\end{tabular}

Table 13. Charpy-V Impact properties at $25^{\circ} \mathrm{C}$ for Inconel 617 and 618 after various treatments

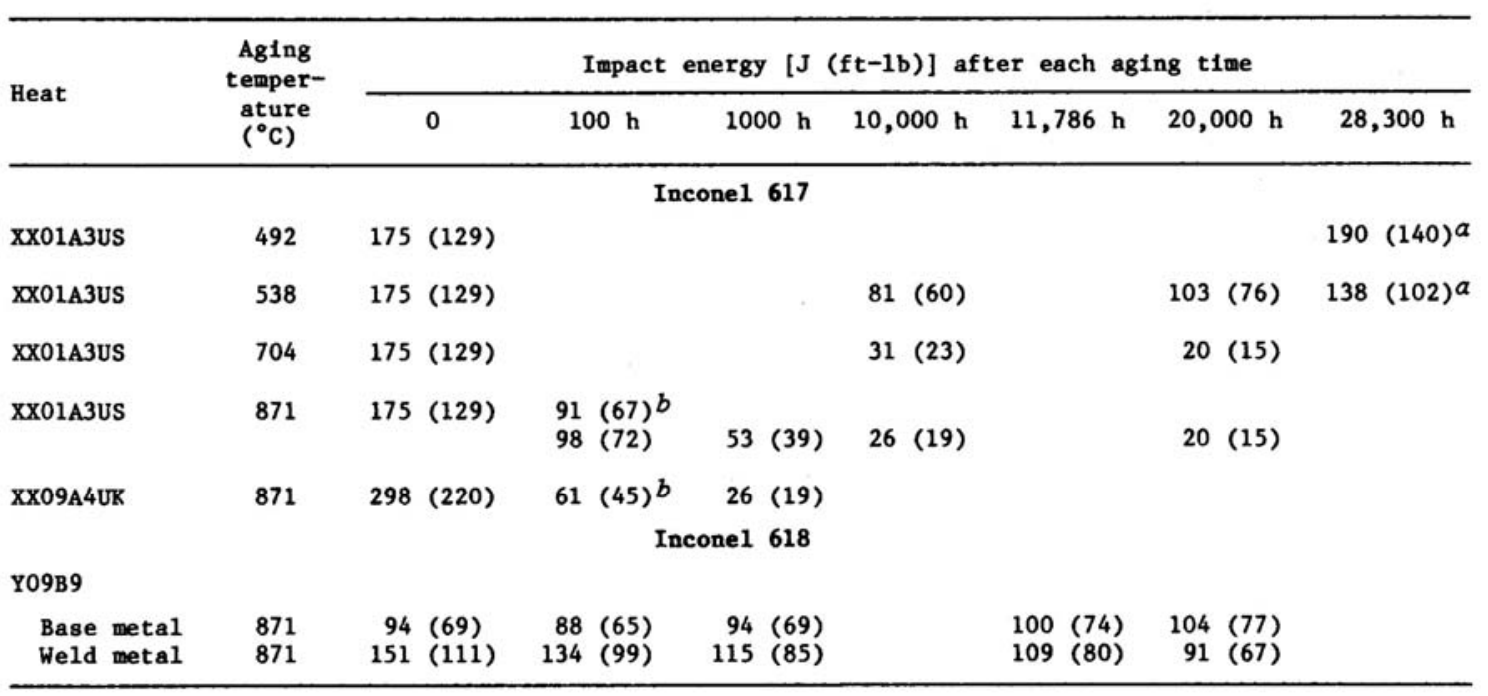

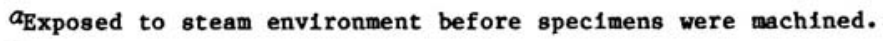

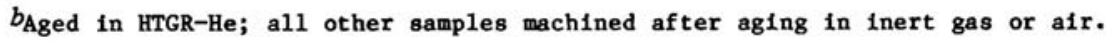


ASSESSMENT OF EXISTING ALLOY 617 DATA FOR GEN IV NUCLEAR REACTOR APPLICATIONS

\begin{tabular}{|l|l|l|l|}
\hline Data ID: & McCoy285T14 & Data Class: & 5 \\
\hline Project: & ORNL US DOE HTGR Project & Reference: & McCoy 1985 \\
\hline $\begin{array}{l}\text { Comment: Material was aged in steam in the form of blocks before specimens were } \\
\text { machined. Therefore the aging environment is considered inert. }\end{array}$ \\
\hline
\end{tabular}

Table 14. Results of creep tests on Inconel 617 base metal

\begin{tabular}{|c|c|c|c|c|c|c|c|c|c|c|c|c|c|c|}
\hline \multirow{2}{*}{ Test } & \multirow{2}{*}{ Heat ${ }^{a}$} & \multicolumn{2}{|c|}{ Stress } & \multirow{2}{*}{$\begin{array}{c}\text { Environ- } \\
\text { ment }\end{array}$} & \multirow{2}{*}{ Condition $b$} & \multicolumn{3}{|c|}{$\begin{array}{l}\text { Time to Indicatend } \\
\text { creep atrain (h) }\end{array}$} & \multirow{2}{*}{$\begin{array}{l}\text { Time to } \\
\text { tertiary } \\
\text { creep } \\
\text { (h) }\end{array}$} & \multirow{2}{*}{$\begin{array}{l}\text { Rupture } \\
\text { or teat } \\
\text { (h) }\end{array}$} & \multirow{2}{*}{$\begin{array}{l}\text { Steady-atate } \\
\text { creep rate } \\
\left(h^{-1}\right)\end{array}$} & \multicolumn{2}{|c|}{ Elongat ion (z) } & \multirow{2}{*}{$\begin{array}{l}\text { Reduct for } \\
\text { of area } \\
\text { (z) }\end{array}$} \\
\hline & & (MPa) & (kst) & & & 12 & $2 z$ & $5 x$ & & & & Loading & Creep & \\
\hline \multicolumn{15}{|c|}{ Tested at $593^{\circ} \mathrm{C}\left(1100^{\circ} \mathrm{F}\right)$} \\
\hline 22188 & $\Lambda$ & 414 & 60 & A1r $\mathbf{r}$ & As recetved & 20 & & 3,350 & 3,445 & 3,570 & $4.8 \mathrm{E}-6$ & 16.0 & 6.9 & 18.2 \\
\hline 20531 & $\mathrm{~A}$ & 414 & 60 & He & As recetved & 380 & 1,700 & & 4,534 & 4,534 & $.4 .9 \mathrm{E}-6$ & 18.0 & 3.7 & 21.3 \\
\hline 20521 & $\mathbf{A}$ & 414 & 60 & He & $593 / 10,000 / \mathrm{He}$ & 500 & & & & 548 & $1.0 \mathrm{E}-5$ & & 2.2 & 22.0 \\
\hline 22645 & $\hat{A}$ & 414 & 60 & He & $593 / 20,000 / \mathrm{He}$ & 2,000 & & & 2,600 & 2,676 & $2.4 \mathrm{E}-6$ & 1.3 & 3.9 & 12.0 \\
\hline 22200 & $A$ & 345 & so & He & $593 / 10,000 / \mathrm{He}$ & & & & & 5,629 & $3.5 \mathrm{E}-7$ & 4.4 & 5.5 & 14.4 \\
\hline 23088 & B & 345 & so & Alr & As recelved & & & & & 2,107 & $1.4 \mathrm{E}-6$ & 9.3 & 3.4 & 15.2 \\
\hline \multicolumn{15}{|c|}{ Teated at $649^{\circ} \mathrm{C}\left(1200^{\circ} \mathrm{Y}\right)$} \\
\hline 19106 & A & 414 & 60 & He & As received & 0.5 & 5 & 145 & 168 & 186 & $1.6 \mathrm{z}-4$ & 15.3 & 7.5 & 16.1 \\
\hline 21589 & $\ddot{A}$ & 345 & so & A1r & $482 / 28,300 /$ steas & 280 & 1,100 & & 1,700 & 1,813 & $1.38-5$ & 8.4 & 5.6 & 13.5 \\
\hline 21610 & $\hat{A}$ & 345 & 50 & Afr & $538 / 28,300 /$ Stean & 189 & 837 & & & 1,063 & $2.4 \mathrm{E}-5$ & 6.8 & 6.5 & 13.5 \\
\hline 19182 & $\hat{A}$ & 276 & 40 & $\mathrm{He}$ & As recelved & 5,000 & 8,600 & 15,100 & 8,000 & 16,718 & $2.0 \mathrm{E}-6$ & 0.03 & 9.3 & 14.9 \\
\hline 18483 & A & 276 & 40 & $\mathrm{He}$ & As recelved & 7,600 & & & & 15,850 & $1.1 \mathrm{E}-6$ & 2.5 & 6.1 & 8.3 \\
\hline 18440 & 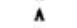 & 276 & 40 & Afr & As recelved & 8,850 & 16,480 & 25,510 & 16,000 & 25,566 & $9.3 \mathrm{~B}-7$ & 1.5 & 6.4 & 15.7 \\
\hline 19182 & $A$ & 207 & 30 & $\mathrm{Be}$ & As received & & & & & $26,515^{\circ}$ & $9.9 \mathrm{E}-8$ & 0.3 & 0.3 & \\
\hline 23085 & B & 276 & 40 & A1r & As recelved & & & & & 1,121 & $1.9 \mathrm{x}-6$ & 1.5 & 1.4 & 4.5 \\
\hline 23339 & B & 276 & 40 & Air & As recelved & & & & & 667 & $3.1 \mathrm{E}-6$ & 4.6 & 1.9 & 10.0 \\
\hline 22751 & B & 276 & 40 & He & As recelved & & & & & 1,208 & $3.0 \mathrm{E}-6$ & 2.3 & 0.8 & 10.6 \\
\hline \multicolumn{15}{|c|}{ Tested at $704^{\circ} \mathrm{C}\left(1300^{\circ} \mathrm{r}\right)$} \\
\hline 22742 & $A$ & 276 & 40 & He & As received & 7 & 13 & 47 & 212 & 329 & $5.1 \mathrm{E}-4$ & 3.5 & 33.0 & 45.2 \\
\hline 21579 & $\mathbf{A}$ & 276 & 40 & He & $704 / 10,000 / \mathrm{He}$ & & & & & 584 & & & 9.1 & 17.8 \\
\hline 20526 & $\hat{A}$ & 207 & 30 & He & $704 / 10,000 /$ He & 3,450 & 5,050 & & 3,800 & 5,424 & $2.0 \mathrm{E}-6$ & & 3.7 & 2.4 \\
\hline 22180 & $\ddot{A}$ & 207 & 30 & $\mathrm{He}$ & $704 / 20,000 /$ Inert & 925 & 2,285 & 4,529 & 2,500 & 5,585 & $7.1 \mathrm{E}-7$ & 0.2 & 10.9 & 14.3 \\
\hline 23342 & B & 207 & 30 & Afr & As received & & & & & 2,621 & $1.2 \mathrm{E}-6$ & 0.4 & 1.2 & 1.6 \\
\hline 22753 & B & 207 & 30 & मе & As received & 6,200 & & & 6,700 & 7,034 & $1.2 \mathrm{E}-6$ & 0.4 & 2.5 & 3.6 \\
\hline 22760 & B & 172 & 25 & He & As recelved & 9,600 & 16,400 & & & $18,526^{d}$ & $6.5 \mathrm{E}-6$ & 0.5 & 2.9 & \\
\hline \multicolumn{15}{|c|}{ Tested at $760^{\circ} \mathrm{C}\left(1400^{\circ} \mathrm{V}\right)$} \\
\hline 17563 & $\mathbf{A}$ & 172 & 25 & Be & As received & 9 & 20 & 57 & 105 & . 280 & $8.9 \mathrm{~g}-4$ & 0.2 & 53.6 & 74.1 \\
\hline 23091 & $\mathbf{A}$ & 138 & 20 & A1r $r$ & As recelved & 10 & 28 & 123 & 780 & 1,305 & $2.2 \mathrm{E}-4$ & & 48.5 & 56.9 \\
\hline 21578 & $\mathbf{A}$ & 138 & 20 & He & $538 / 28,300 /$ steas & 209 & 440 & 870 & 550 & 1,762 & $3.9 \mathrm{E}-5$ & 0.06 & 24.5 & 46.2 \\
\hline 20032 & $\vec{A}$ & 103 & 15 & A1r & As recelved & 125 & 750 & 3,500 & 17,000 & 20,702 & $1.0 \mathrm{E}-5$ & & 28.0 & 31.9 \\
\hline 22754 & B & 172 & 25 & Be & As recelved & 513 & 740 & 935 & 517 & 948 & $1.3 \mathrm{~g}-3$ & & 5.4 & 9.4 \\
\hline 22755 & B & 138 & 20 & He & As recelved & & & & & 4,585 & & 0.3 & 9.4 & 21.8 \\
\hline \multicolumn{15}{|c|}{ Teoted at $871^{\circ} \mathrm{C}\left(1600^{\circ} \mathrm{r}\right)$} \\
\hline 21611 & $\boldsymbol{A}$ & 69 & 10 & A1r & As received & 5 & 20 & & & 576 & & & 45.1 & 44.7 \\
\hline 20492 & $\hat{A}$ & 48 & 7 & A1r & Ao recelved & 240 & 500 & 1,200 & 2,400 & 4,800 & $4.18-5$ & 0.09 & 26.2 & 28.3 \\
\hline 20545 & $\hat{A}$ & 48 & 7 & $\mathrm{He}$ & As recelved & 2,500 & 3,500 & & 3,000 & 3,562 & $1.18-4$ & & 11.8 & 18.4 \\
\hline 20544 & A & 48 & 7 & He & $871 / 20,000 /$ Inert & 40 & 92 & 345 & 2,200 & 2,282 & $5.7 \mathrm{z}-5$ & & 21.3 & 28.7 \\
\hline 22185 & $\ddot{a}$ & 48 & 7 & He & $871 / 20,000 / \mathrm{He}$ & 40 & 100 & 320 & 1,880 & 1,933 & $5.7 \mathrm{E}-5$ & & 18.8 & 24.0 \\
\hline 19393 & $A$ & 35 & 5 & Be & Ao recelved & 3,200 & 5,848 & 13,875 & 26,800 & 29,544 & $3.78-6$ & 0.002 & 16.5 & 22.3 \\
\hline 19761 & $A$ & 35 & 5 & A1r & As recelved & 3,100 & 4,780 & 8,670 & 23,200 & 34,231 & $7.9 \mathrm{E}-6$ & 0.07 & 30.3 & 23.3 \\
\hline 23827 & B & 48 & 7 & Be & As recelved & 2,900 & 3,700 & 5,670 & 2,300 & 7,543 & $1.6 \mathrm{E}-4$ & 0.02 & 11.4 & 11.6 \\
\hline 21603 & $B$ & 21 & 3 & Aer & An received & & & & & $26,117^{*}$ & $1.9 \mathrm{E}-7$ & 0.01 & 1.3 & \\
\hline 21602 & B & 21 & 3 & $\mathrm{He}$ & Ao recelved & & & & & $25,699^{\circ}$ & $2.6 \mathrm{E}-7$ & & 0.27 & \\
\hline
\end{tabular}

$a_{A}$ - heat XX01A3US; B - heat $0 \times 09$ AUUK.

${ }^{b}$ Aging temperature $\left({ }^{\circ} \mathrm{C}\right) /$ aging tiae $(\mathrm{h}) / \mathrm{ag}$ ing environment.

a Teat reloaded at a higher stress.

deat in progress.

ODiecontinued before fallure. 


\begin{tabular}{|l|l|l|l|}
\hline Data ID: & McCoy285T15 & Data Class: & 5 \\
\hline Project: & ORNL US DOE HTGR Project & Reference: & McCoy 1985 \\
\hline Comment:
\end{tabular}

Table 15. Results of creep tests on Inconel 617 weld metal in helium environment

(Weld metal heat XX09A9UK)

\begin{tabular}{|c|c|c|c|c|c|c|c|c|c|c|c|}
\hline \multirow{2}{*}{ Test } & \multicolumn{2}{|c|}{ Stress } & \multicolumn{3}{|c|}{$\begin{array}{l}\text { Time to Indicated } \\
\text { creep strain (h) }\end{array}$} & \multirow{2}{*}{$\begin{array}{l}\text { Time to } \\
\text { tertiary } \\
\text { creep } \\
\text { (h) }\end{array}$} & \multirow{2}{*}{$\begin{array}{l}\text { Rupture } \\
\text { or test } \\
\text { (h) }\end{array}$} & \multirow{2}{*}{$\begin{array}{l}\text { Steady-state } \\
\text { creep rate } \\
\left(\mathrm{h}^{-1}\right)\end{array}$} & \multicolumn{2}{|c|}{ Elongation (z) } & \multirow{2}{*}{$\begin{array}{l}\text { Reduction } \\
\text { of area } \\
\text { (z) }\end{array}$} \\
\hline & (MPa) & (ks1) & 17 & $2 \pi$ & $5 \%$ & & & & Loading & Creep & \\
\hline \multicolumn{12}{|c|}{ Tested at $593^{\circ} \mathrm{C}\left(1100^{\circ} \mathrm{P}\right)$} \\
\hline 20515 & $\begin{array}{l}345 \\
414\end{array}$ & $\begin{array}{l}50 \\
60\end{array}$ & & & & & $\begin{array}{c}13,726^{a} \\
1,296\end{array}$ & $1.2 \mathrm{E}-7$ & $\begin{array}{l}0.6 \\
0.09\end{array}$ & $\begin{array}{l}0.4 \\
0.1\end{array}$ & \\
\hline 23071 & 414 & 60 & & & & & 2,034 & $1.3 \mathrm{E}-6$ & 0.72 & 2.0 & 5.1 \\
\hline \multicolumn{12}{|c|}{ Tested at $649^{\circ} \mathrm{C}\left(1200^{\circ} \mathrm{P}\right)$} \\
\hline $\begin{array}{l}21399 \\
20513\end{array}$ & $\begin{array}{l}345 \\
276\end{array}$ & $\begin{array}{l}50 \\
40\end{array}$ & 525 & & & & $\begin{array}{r}526 \\
3,467\end{array}$ & $\begin{array}{l}8.1 \text { E-6 } \\
5.4 \mathrm{E}-7\end{array}$ & $\begin{array}{l}0.1 \\
0.1\end{array}$ & $\begin{array}{l}2.6 \\
2.3\end{array}$ & $\begin{array}{l}3.3 \\
1.4\end{array}$ \\
\hline 20553 & 242 & 35 & 15,400 & & & & 18,182 & $4.7 \mathrm{E}-7$ & 0.2 & 1.8 & 2.9 \\
\hline \multicolumn{12}{|c|}{ Tested at $704^{\circ} \mathrm{C}\left(1300^{\circ} \mathrm{F}\right)$} \\
\hline 22741 & 276 & 40 & 775 & & & 785 & 790 & $8.0 \mathrm{E}-6$ & 0.5 & 4.4 & 5.9 \\
\hline 22763 & 207 & 30 & 4,250 & 10,500 & 14,900 & 6,000 & 14,978 & $5.5 \mathrm{E}-7$ & 0.04 & 7.7 & 14.1 \\
\hline \multicolumn{12}{|c|}{ Tested at $760^{\circ} \mathrm{C}\left(1400^{\circ} \mathrm{F}\right)$} \\
\hline 20735 & 138 & 20 & 2,900 & 3,750 & 4,750 & 2,600 & 5,190 & $1.8 \mathrm{E}-6$ & & 17.1 & 43.5 \\
\hline \multicolumn{12}{|c|}{ Tested at $871^{\circ} \mathrm{C}\left(1600^{\circ} \mathrm{p}\right)$} \\
\hline 20533 & 69 & 10 & 1,467 & 1,570 & & & 1,623 & & 0.07 & 10.7 & 21.0 \\
\hline
\end{tabular}

\begin{tabular}{|l|l|l|l|}
\hline Data ID: & McCoy285T16 & Data Class: & 5 \\
\hline Project: & ORNL US DOE HTGR Project & Reference: & McCoy 1985 \\
\hline Comment:
\end{tabular}

Table 16. Results of creep tests on Inconel 617 transverse welds in helium environment

(Base plate heat XX14A6UK, weld metal XX09A9UK)

\begin{tabular}{|c|c|c|c|c|c|c|c|c|c|c|}
\hline \multirow{2}{*}{ Test } & \multirow{2}{*}{ Condition ${ }^{a}$} & \multicolumn{3}{|c|}{$\begin{array}{l}\text { Time to Indicated } \\
\text { creep strain (h) }\end{array}$} & \multirow{2}{*}{$\begin{array}{l}\text { Time to } \\
\text { tertiary } \\
\text { creep } \\
\text { (h) }\end{array}$} & \multirow{2}{*}{$\begin{array}{l}\text { Rupture } \\
\text { or test } \\
\text { (h) }\end{array}$} & \multirow{2}{*}{$\begin{array}{l}\text { Steady-state } \\
\text { creep rate } \\
\left(h^{-1}\right)\end{array}$} & \multicolumn{2}{|c|}{ Elongation (z) } & \multirow{2}{*}{$\begin{array}{c}\text { Reduction } \\
\text { of area } \\
\text { (z) }\end{array}$} \\
\hline & & 17 & 27 & $5 \%$ & & & & Loading & Creep & \\
\hline \multicolumn{11}{|c|}{ Tested at $593^{\circ} \mathrm{C}\left(1100^{\circ} \mathrm{F}\right)$ and $414 \mathrm{kPa}(60 \mathrm{ks} 1)$} \\
\hline \multirow[t]{2}{*}{20522} & $593 / 10,000 / \mathrm{He}$ & & & & & 645 & & & 1.7 & 5.2 \\
\hline & & & Tested & at $704^{\circ}$ & C $\left(1300^{\circ} \mathrm{F}\right)$ & and $207 \mathrm{r}$ & a $(30 \mathrm{ks1})$ & & & \\
\hline 20528 & $704 / 10,000 / \mathrm{He}$ & 4,830 & 6,380 & & 5,100 & 6,932 & $2.6 \mathrm{E}-6$ & 0.3 & 3.7 & 17.1 \\
\hline 21612 & $704 / 20,000 / \mathrm{He}$ & 1,100 & 3,250 & 5,470 & 3,025 & 5,496 & $3.7 \mathrm{E}-6$ & 0.0 & 6.5 & 17.6 \\
\hline
\end{tabular}

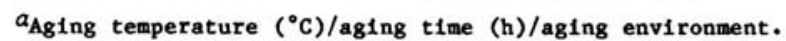




\section{$\underline{\text { GE-HTGR Data }}$}

The GE-HTGR data source contains the data generated over a period of eight years from 1978 to 1986 for the HTGR project by General Electric Company (GE) under DOE contract No. DE-AC03-80ET34034. The source document [Baldwin 1986] is a summary report on fatigue and creep test results on Alloy 617 and Alloy $800 \mathrm{H}$. The report includes creep properties data from 36 creep tests at temperatures of 750, 850, 950, 1050 and $1100^{\circ} \mathrm{C}\left(1382,1552,1742,1922\right.$ and $\left.2012^{\circ} \mathrm{F}\right)$; creep-fatigue properties data from 7 creep-fatigue test at $950^{\circ} \mathrm{C}\left(1742^{\circ} \mathrm{F}\right)$; fatigue properties data from 40 fatigue tests at temperatures of 850 and $950^{\circ} \mathrm{C}\left(1562\right.$ and $\left.1742^{\circ} \mathrm{F}\right)$, which consist of 27 low cycle fatigue tests conducted under strain control and 13 high cycle fatigue tests conducted under load control. A summary of the tests that generated the GE-HTGR data is given in Table 15. The creep properties include the elastic and plastic components of the loading strains, times to $0.1 \%, 0.2 \%, 0.5 \%, 1 \%, 2 \%$, and $5 \%$ total strain, time to onset of tertiary creep, time to $0.2 \%$ offset tertiary creep, minimum creep rate, total creep strain, reduction in area, creep rupture time, and creep rupture stress. The fatigue and creep-fatigue properties include fatigue life data and strain-life curves. Based on the Gen IV Materials Handbook Data Classification Criteria, the data are classified as Class 5.

Table 15: Summary of GE-HTGR data on Alloy 617 (no weld data)

\begin{tabular}{|c|c|c|c|c|c|c|c|}
\hline \multirow{2}{*}{$\begin{array}{l}\text { Test } \\
\text { Type }\end{array}$} & \multirow{2}{*}{$\begin{array}{c}\text { Sample } \\
\text { Treatment }\end{array}$} & \multirow{2}{*}{$\begin{array}{c}\text { Test } \\
\text { Evironment }\end{array}$} & \multicolumn{5}{|c|}{ Test Temperature ${ }^{\circ} \mathrm{C}$} \\
\hline & & & 750 & 850 & 950 & 1050 & 1100 \\
\hline \multirow{2}{*}{ Creep } & \multirow{2}{*}{ Solution Annealed } & Air & 1 & 10 & 10 & 3 & 1 \\
\hline & & $\mathrm{He}$ & 1 & 3 & 3 & 4 & \\
\hline \multirow{3}{*}{$\begin{array}{l}\text { Low Cycle } \\
\text { Fatigue }\end{array}$} & He Aging* & $\mathrm{He}$ & & 3 & 3 & & \\
\hline & \multirow{2}{*}{ Solution Annealing } & Air & & 3 & 7 & & \\
\hline & & $\mathrm{He}$ & & 1 & 10 & & \\
\hline \multirow{3}{*}{$\begin{array}{c}\text { High Cycle } \\
\text { Fatigue }\end{array}$} & He Aging* & $\mathrm{He}$ & & 3 & 3 & & \\
\hline & \multirow{2}{*}{ Solution Annealing } & Air & & 1 & & & \\
\hline & & $\mathrm{He}$ & & 3 & 3 & & \\
\hline Creep-Fatigue & Solution Annealing & Air & & & 7 & & \\
\hline
\end{tabular}

* Aged in simulated HTGR helium for 6000 hours at the same temperature as the test temperature

Table 16: Compositions (wt. \%) of the Alloy 617 heats produced for the GE-HTGR data [Baldwin486T3]

\begin{tabular}{|c|c|c|c|c|c|c|c|c|c|c|c|c|c|c|}
\hline Heat & & Ni & Cr & Co & Mn & Mo & Fe & A1 & Si & C & S & Ti & Cu & B \\
\hline \multirow{2}{*}{ XX14A6UK } & Max & bal & 21.74 & 12.32 & 0.02 & 8.91 & 0.53 & 1.11 & 0.18 & $0.060^{\mathrm{a}}$ & 0.002 & 0.30 & 0.11 & 0.003 \\
\cline { 2 - 12 } & Min & bal & 23.12 & 12.14 & 0.034 & 9.09 & 0.53 & 1.31 & 0.257 & $0.054^{\mathrm{c}}$ & - & 0.363 & - & - \\
\hline \multirow{2}{*}{ XX63A8UK } & Max & bal & 22.30 & 12.10 & 0.06 & 9.27 & 1.02 & 1.07 & 0.19 & $0.07^{\mathrm{a}}$ & 0.001 & 0.37 & 0.09 & 0.003 \\
\cline { 2 - 12 } & Min & bal & 21.8 & 11.8 & 0.049 & 9.3 & 0.93 & 0.92 & 0.19 & $0.075^{\mathrm{c}}$ & - & 0.39 & 0.092 & 0.005 \\
\hline
\end{tabular}

a. From vendor certificated values, c. Values determined at GE 
All the GE-HTGR data were generated at GE's High Temperature Reactor Materials Testing Laboratory from two commercial heats produced by Huntington Alloy Product Division. The compositions of the heats are given in Table 16, and their product characterization is listed in Table 17. It is noted that Heat XX14A6UK was also tested to generate the previously discussed ORNL-HTGR data.

Table 17: Product characterization of the Alloy 617 heats produced by Huntington Alloy Product Division for the GE-HTGR data [Baldwin486T2]

\begin{tabular}{|c|c|c|c|c|}
\hline Heat No. & $\begin{array}{c}\text { Product } \\
\text { Form }\end{array}$ & $\begin{array}{c}\text { Heat } \\
\text { Treatment }\end{array}$ & Grain Size $^{\mathrm{a}}$ & Grain Size $^{\mathrm{b}}$ \\
\hline XX14A6UK & $\begin{array}{c}16 \mathrm{~mm}\left(5 / 8^{\prime \prime}\right) \\
\text { plate }\end{array}$ & $\begin{array}{c}\text { Solution Annealing } \\
1204^{\circ} \mathrm{C}\end{array}$ & 00 & 0.5 \\
\hline XX63A8UK & $\begin{array}{c}44.5 \mathrm{~mm}\left(1.75^{\prime \prime}\right) \\
\text { round bar }\end{array}$ & $\begin{array}{c}\text { Solution Annealing } \\
1177^{\circ} \mathrm{C}\end{array}$ & 4.5 & - \\
\hline
\end{tabular}

a. measured by vendor, b. measured by GE

The simulated HTGR helium for generating the GE-HTGR data was premixed and flowed to the testing environmental chamber at a nominal flow rate of $2 \mathrm{std} \mathrm{L} / \mathrm{min}$. The helium composition supplied to the test chamber was controlled within the limits as shown in Table 18. During normal operation, the total gas pressure in the test chamber was 202,650 $\mathrm{Pa}(2 \mathrm{~atm})$ absolute. The estimated gas chemistries at the test specimen position are given in Table 19. The estimation was based on an assumption that $50 \%$ of the total change in gas composition between the chamber inlet and outlet had occurred before the test gas reached the specimen located at the radial and longitudinal center of the chamber uniform hot zone.

Table 18: Helium composition in the environmental test chamber for the GE-HTGR data [Baldwin486P5]

\begin{tabular}{|c|c|c|c|c|c|c|c|c|c|c|c|}
\hline \multicolumn{2}{|c|}{$\mathrm{H}_{2}$} & \multicolumn{2}{c|}{$\mathrm{H}_{2} \mathrm{O}$} & \multicolumn{2}{c|}{$\mathrm{CO}$} & \multicolumn{2}{c|}{$\mathrm{CO}_{2}$} & \multicolumn{2}{c|}{$\mathrm{CH}_{4}$} & \multicolumn{2}{c|}{$\mathrm{N}_{2}$} \\
\hline $\mathrm{Pa}$ & $\mu \mathrm{atm}$ & $\mathrm{Pa}$ & $\mu \mathrm{atm}$ & $\mathrm{Pa}$ & $\mu \mathrm{atm}$ & $\mathrm{Pa}$ & $\mu \mathrm{atm}$ & $\mathrm{Pa}$ & $\mu \mathrm{atm}$ & $\mathrm{Pa}$ & $\mu \mathrm{atm}$ \\
\hline 40.53 & 400 & 0.20 & 2 & 4.05 & 40 & 0.02 & 0.2 & 2.0 & 20 & 0.61 & 6 \\
\pm 7.5994 & \pm 75 & \pm 0.0760 & \pm 0.75 & \pm 0.7599 & \pm 7.5 & \pm 0.0051 & \pm 0.05 & \pm 0.7599 & \pm 7.5 & \pm 0.3040 & \pm 3 \\
\hline
\end{tabular}

Table 19: Estimated local gas compositions at the position of the test specimens for the GE-HTGR creep tests in simulated HTGR helium [Baldwin486]

\begin{tabular}{|c|c|c|c|c|c|c|}
\hline \multirow{2}{*}{$\begin{array}{c}\text { Temp. } \\
{ }^{\circ} \mathrm{C}\end{array}$} & \multicolumn{7}{|c|}{ Impurity Partial Pressure $(\mathrm{Pa})$} \\
\cline { 2 - 7 } & $\mathrm{H}_{2}$ & $\mathrm{H}_{2} \mathrm{O}$ & $\mathrm{CO}$ & $\mathrm{CO}_{2}$ & $\mathrm{CH}_{4}$ & $\mathrm{~N}_{2}$ \\
\hline 750 & 40.1 & 0.18 & 4.01 & 0.0195 & 2.00 & 0.6 \\
\hline 850 & 40.2 & 0.07 & 4.07 & 0.019 & 1.96 & 0.6 \\
\hline 950 & 40.3 & $<0.01$ & 4.19 & 0.13 & 1.85 & 0.6 \\
\hline 1050 & 40.5 & $<0.001$ & 4.26 & $<0.010$ & 1.62 & 0.6 \\
\hline
\end{tabular}

It is worth mentioning that after the GE-HTGR testing program was terminated, a few of the environmental chambers and some testing accessories were shipped to ORNL. Some of these parts can still be used for the Gen IV materials testing. 


\section{ORIGINAL GE - HTGR DATA}

Due to large size of the original document, only summary data are presented. For detailed data, the original source document must be used. 
ASSESSMENT OF EXISTING ALLOY 617 DATA FOR GEN IV NUCLEAR REACTOR APPLICATIONS

\begin{tabular}{|l|l|l|l|}
\hline Data ID: & Baldwin486T4 & Data Class: & 4 \\
\hline Data Source: & GE HTGR Project & Reference: & Baldwin 1986 \\
\hline Comment: &
\end{tabular}

TABLE 4

CREEP AND RUPTURE. TEST RESULTS FOR INCONEL 617

\begin{tabular}{|c|c|c|c|c|c|c|c|c|c|c|c|c|c|}
\hline $\begin{array}{c}\text { Specimen } \\
\text { No. }\end{array}$ & 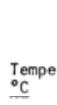 & $\begin{array}{l}\text { ature } \\
\left({ }^{\circ} \mathrm{F}\right) \\
\end{array}$ & $\begin{array}{c}\text { Str } \\
\mathrm{MPa}\end{array}$ & $\begin{array}{l}\text { ess } \\
\text { ksi }\end{array}$ & Environment & $\begin{array}{l}\text { Time to } \\
\text { 18 Total } \\
\text { Strain } \\
\mathrm{h} \\
\end{array}$ & $\begin{array}{l}\text { Time to } \\
\text { Initiate } \\
\text { Stage II } \\
\mathrm{h}\end{array}$ & $\begin{array}{l}\text { Minimum } \\
\text { Creep } \\
\text { Rate } \\
8 / h \\
\end{array}$ & $\begin{array}{l}\text { Time to } \\
\text { Onset of } \\
\text { Tertiary } \\
\text { Creep } \\
\quad \text { h } \\
\end{array}$ & $\begin{array}{l}\text { Time to } \\
0.28 \text { off set } \\
\text { Tertiary } \\
\text { Creep } \\
\mathrm{h} \\
\end{array}$ & $\begin{array}{l}\text { Rupture } \\
\text { Life } \\
\mathrm{h} \\
\end{array}$ & $\begin{array}{c}\text { Elongation } \\
\end{array}$ & $\begin{array}{l}\text { Reduction } \\
\text { in Area } \\
\quad 8 \\
\end{array}$ \\
\hline \multicolumn{14}{|l|}{ Heat No. 1} \\
\hline TAOOCRO & -750 & $(1382)$ & 200 & 29.0 & Air & 97 & - & - & - & - & 625 & 19.6 & 24.8 \\
\hline 1 A00CR27 & $\begin{array}{l}750 \\
850\end{array}$ & $(1382)$ & 159 & 23.0 & $\begin{array}{l}\text { Helium } \\
\text { Afr }\end{array}$ & 24.5 & 120 & 0.0136 & 490 & 533 & $\begin{array}{r}750 \\
1126\end{array}$ & 17.5 & 23.2 \\
\hline 1AOOCRO2 & 850 & $(1552)$ & 72.4 & 10.5 & Air & 20.5 & - & - 000 & & & $\begin{array}{r}1126 \\
1126\end{array}$ & 58.3 & 60.9 \\
\hline 1AOOCROB & 850 & $(1562)$ & 56.6 & 8.20 & Air & 1100 & 70 & 0.00042 & 142 & 785 & 12207 & 43.1 & 75.2 \\
\hline 1AOOCR11 & 850 & $(1562)$ & 52.4 & 7.60 & Helium & 990 & 60 & $\begin{array}{l}0.000605 \\
0.0535\end{array}$ & $\begin{array}{l}530 \\
215\end{array}$ & 882 & 17146 & 41.2 & 44.0 \\
\hline $\begin{array}{l}\text { 1AOOCR14 } \\
1 \text { AOOCR17 }\end{array}$ & 850 & $\begin{array}{l}(1562) \\
(156)\end{array}$ & 79.3 & 11.5 & Air & 16.5 & $=3.5$ & 0.0410 & $\begin{array}{r}215 \\
20\end{array}$ & 260 & $\begin{array}{l}596 \\
383\end{array}$ & 56.7 & 64.1 \\
\hline & $\begin{array}{l}850 \\
850\end{array}$ & $\begin{array}{l}(1562) \\
(1562)\end{array}$ & $\begin{array}{l}79.3 \\
62.1\end{array}$ & $\begin{array}{l}11.5 \\
9.00\end{array}$ & $\begin{array}{l}\text { Helium } \\
\text { Helium }\end{array}$ & -21 & 3.5 & 0.0410 &.$^{20}$ & .43 & $\begin{array}{r}383 \\
4143\end{array}$ & 36.7 & 42.7 \\
\hline $\begin{array}{l}\text { 1A00CR25 } \\
\text { 1A00CR206 }\end{array}$ & $\begin{array}{l}850 \\
850\end{array}$ & $\begin{array}{l}(1562) \\
(1562)\end{array}$ & $\begin{array}{l}2.1 \\
64.8\end{array}$ & 9.40 & $\begin{array}{l}\text { Melrom } \\
\text { Air }\end{array}$ & 13.8 & - & - & $=$ & $:$ & $\begin{array}{l}\begin{array}{l}4143 \\
1046\end{array} \\
\end{array}$ & $\begin{array}{l}46.7 \\
68.7\end{array}$ & 56.0 \\
\hline 1 A00CRO4 & 950 & (1742) & $\begin{array}{l}48.0^{\circ} \\
48 .\end{array}$ & 7.00 & Air & 6.7 & - & - & - & : & 334 & $\begin{array}{l}68.7 \\
46.9\end{array}$ & $\begin{array}{l}59.1 \\
46.9\end{array}$ \\
\hline 1AOOCROS & 950 & (1742) & 25.5 & 3.70 & Air & $2750^{\circ}$ & - & 0.000119 & $=$ & - & 22188 & & \\
\hline $1 A 00 C R 12$ & 950 & (1742) & 24.1 & 3.50 & Helium & 4400 & 1900 & 0.0000446 & 4300 & 5060 & 28920 & 25.8 & 20.4 \\
\hline 1A00CR15 & 950 & (1742) & 37.9 & 5.50 & Air & 70 & -00 & $-0000+90$ & - & - & 723 & 34.9 & 33.9 \\
\hline 1A0OCR18 & 950 & (1742) & $\begin{array}{r}37.9 \\
37.9\end{array}$ & $\begin{array}{l}5.50 \\
5.50\end{array}$ & Helium & 440 & 10 & 0.000285 & 26 & 315 & 2025 & 28.1 & $\begin{array}{l}33.9 \\
46.1\end{array}$ \\
\hline 1AOOCR21 & 950 & (1742) & 29.0 & $\begin{array}{l}3.20 \\
4.20\end{array}$ & Helium & 1630 & .10 & 0.000175 & 185 & 1240 & 3173 & 8.5 & $\begin{array}{r}46.1 \\
9.4\end{array}$ \\
\hline $1 \mathrm{AOOCRO}_{4}$ & 1050 & (1922) & 20.7 & 3.00 & Air & 125 & - & -0001 & - & -8 & 1146 & 23.2 & $\begin{array}{r}9.4 \\
35.4\end{array}$ \\
\hline 1A00CR10 & 1050 & (1922) & 10.7 & 1.55 & Air & 3100 & 370 & 0.000078 & 370 & 2140 & 8514 & 14.5 & 13.7 \\
\hline 1A00CR16 & 1050 & (1922) & 14.5 & 2.10 & Air & 950 & $=$ & 0.00026 & 305 & 639 & 9285 & 33.0 & 26.6 \\
\hline 1AO0CR19 & 1050 & (1922) & 14.5 & 2.10 & Helium & 380 & 110 & 0.00104 & 325 & 550 & 3752 & 28.3 & 43.8 \\
\hline $1 A 00 C R 22$ & 1050 & (1922) & 9.66 & 1.40 & Helium & 1280 & 110 & 0.000460 & 430 & 1060 & 18580 & 35.2 & 48.7 \\
\hline $1 \mathrm{AOOCR} 23$ & 1050 & (1922) & 9.66 & 1.40 & Helium & 1620 & 480 & 0.000420 & 1060 & 1640 & Disc. & & - \\
\hline 1 AOOCR26 & 1050 & (1922) & 14.5 & 2.10 & Hel ium & 580 & 65 & 0.000680 & 135 & 382 & 5123 & 22.9 & 40.2 \\
\hline 1AOOCROS & 1100 & (2012) & 15.2 & 2.20 & Air & 70 & - & - & - & - & 723 & 34.9 & 33.9 \\
\hline \multicolumn{14}{|c|}{ 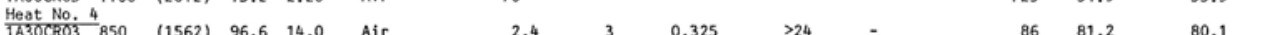 } \\
\hline 1A30CR05 & $\begin{array}{l}850 \\
850\end{array}$ & (1562) & $\begin{array}{l}96.6 \\
66.2\end{array}$ & $\begin{array}{l}17.0 \\
9.60\end{array}$ & Air & $26^{2.4}$ & 8 & $\begin{array}{l}0.325 \\
0.0304\end{array}$ & 330 & 358 & $\begin{array}{r}86 \\
791\end{array}$ & $\begin{array}{l}81.2 \\
42.1\end{array}$ & $\begin{array}{l}80.1 \\
77.6\end{array}$ \\
\hline 06 & 850 & (1562) & 75.9 & 11.0 & Air & 8.5 & 4 & 0.0960 & 80 & 110 & 326 & 78.1 & 81.0 \\
\hline 1A30CR07 & 850 & (156) & 56.6 & 8.20 & Air & 55 & 280 & 0.0 & 850 & 1150 & 22 & 66.8 & 57.0 \\
\hline 1A30CR09 & 850 & (156: & 44.8 & 6.50 & Air & 218 & 500 & 0.00332 & 3400 & 4400 & 7723 & 41.7 & 90.8 \\
\hline $1 \mathrm{~A} 30 \mathrm{CR} 14$ & 850 & (156 & 48.3 & 7.00 & Air & 160 & 850 & & 1800 & 2540 & 4818 & 40.3 & 65.1 \\
\hline 1A30CR04 & 950 & $(1742)$ & 55.2 & 8.00 & Air & 1.3 & 4.5 & 0.612 & $>25$ & & 51 & 56.0 & 66.0 \\
\hline 1A30CR0B & 950 & $(1742)$ & 24.1 & 3.50 & Air & 255 & 15 & 0.00165 & 90 & 195 & 2390 & 40.5 & 30.0 \\
\hline 1A30CR10 & 950 & $(1742)$ & 37.9 & 5.50 & Air & 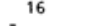 & $=$ & 0.0490 & & & 282 & 38.8 & 47.2 \\
\hline 1A30CR11 & 950 & $(1742)$ & 44.8 & 6.50 & Air & - & - & & - & - & 140 & 55.4 & 50.4 \\
\hline 1A30CR12 & 950 & $(1742)$ & 20.7 & 3.00 & Air & 475 & 60 & 0.00101 & $\begin{array}{r}190 \\
>858\end{array}$ & -372 & 5017 & 36.4 & 28.2 \\
\hline $\begin{array}{l}\text { 1A30CR207 } \\
1 \text { A30CR211 }\end{array}$ & $\begin{array}{l}7950 \\
1950\end{array}$ & $\begin{array}{l}(1742) \\
(1742)\end{array}$ & $\begin{array}{l}31.0 \\
31.0\end{array}$ & $\begin{array}{l}4.50 \\
4.50\end{array}$ & $\begin{array}{l}\text { Air } \\
\text { Air }\end{array}$ & $\begin{array}{l}14 \\
45\end{array}$ & $\begin{array}{l}235 \\
250\end{array}$ & $\begin{array}{l}0.0180 \\
0.0190\end{array}$ & $\begin{array}{r}>858 \\
680\end{array}$ & 788 & $\begin{array}{l}1284 \\
1114\end{array}$ & $\begin{array}{l}41.4 \\
31.5\end{array}$ & $\begin{array}{l}42.6 \\
37.9\end{array}$ \\
\hline
\end{tabular}

\begin{tabular}{|l|l|l|l|}
\hline Data ID: & Baldwin486T8 & Data Class: & 4 \\
\hline Data Source: & GE HTGR Project & Reference: & Baldwin 1986 \\
\hline Comment: & \multicolumn{2}{|l|}{} \\
\hline
\end{tabular}

TABLE 8

INCONEL 617 FATIGUE TEST DATA (HCF), Heat No. XX14A6UK

\begin{tabular}{|c|c|c|c|c|c|}
\hline $\begin{array}{l}\text { Temperature } \\
{ }^{\circ} \mathrm{C}\left({ }^{\circ} \mathrm{F}\right)\end{array}$ & Strain $\underset{\%}{\alpha}$ Range & $\begin{array}{c}\text { Frequency } \\
\mathrm{Hz}\end{array}$ & $\operatorname{Liff}_{\mathrm{N}_{f}}$ & $\begin{array}{l}\text { Stress } \\
\mathrm{MPa}\end{array}$ & $\begin{array}{l}\text { Range } \\
\text { ksi }\end{array}$ \\
\hline \multicolumn{6}{|c|}{ Air Tests: As-received Condition } \\
\hline $850(1562)$ & $0.27^{C}$ & 0.5 & $2.8 \times 10^{7}$ & 413.7 & 60.0 \\
\hline \multicolumn{6}{|c|}{ Simulated HTGR Helium Tests: As-received Condition } \\
\hline $850(1562)$ & $\begin{array}{l}0.18 \\
0.20 \\
0.26\end{array}$ & $\begin{array}{l}30 \\
30 \\
30\end{array}$ & $\begin{array}{l}849000 \\
1.5 \times 10^{6} \\
178800\end{array}$ & $\begin{array}{l}310.3 \\
344.7 \\
413.7\end{array}$ & $\begin{array}{l}45.0 \\
50.0 \\
60.0\end{array}$ \\
\hline $950(1742)$ & $\begin{array}{l}0.11 \\
0.13 \\
0.17\end{array}$ & $\begin{array}{l}30 \\
30 \\
30\end{array}$ & $\begin{array}{l}4.0 \times 10^{6} \\
1.9 \times 10^{6} \\
961000\end{array}$ & $\begin{array}{l}179.3 \\
206.8 \\
248.2\end{array}$ & $\begin{array}{l}26.0 \\
30.0 \\
36.0\end{array}$ \\
\hline
\end{tabular}

Simulated HTGR Hel ium Tests: Test Specimens Exposed 6000 Hours in Simulated HTGR Helium at Same Temperature as Test Temperature

\begin{tabular}{|c|c|c|c|c|}
\hline $850(1562)$ & $\begin{array}{l}0.2 \\
0.24 \\
0.18\end{array}$ & $\begin{array}{l}30 \\
30 \\
30\end{array}$ & $\begin{array}{l}1.2 \times 10^{6} \\
359900 \\
2.42 \times 10^{6}\end{array}$ & $\begin{array}{l}333.7 \\
390.2 \\
317.2\end{array}$ \\
\hline $950 \quad(1742)$ & $\begin{array}{l}0.13 \\
0.19 \\
0.24\end{array}$ & $\begin{array}{l}30 \\
30 \\
30\end{array}$ & $\begin{array}{l}1.13 \times 10^{6} \\
126000 \\
42800\end{array}$ & $\begin{array}{l}206.8 \\
275.8 \\
317.2\end{array}$ \\
\hline
\end{tabular}

$\mathrm{a}=$ Reported values determined from stress strain curves.

$b=N_{f}$ is the number of cycles to total separation.

$c=$ Extensometer in place for 300 cycles. 
ASSESSMENT OF EXISTING ALLOY 617 DATA FOR GEN IV NUCLEAR REACTOR APPLICATIONS

\begin{tabular}{|l|l|l|l|}
\hline Data ID: & Baldwin486T9 & Data Class: & 4 \\
\hline Data Source: & GE HTGR Project & Reference: & Baldwin 1986 \\
\hline Comment: &
\end{tabular}

TABLE 9

INCONEL 617 FATIGUE TEST DATA (LCF), Heat No. XX14A6UK

\begin{tabular}{|c|c|c|c|c|c|c|}
\hline \multirow[b]{2}{*}{$\begin{array}{l}\text { Temperature } \\
{ }^{\circ} \mathrm{C}\left({ }^{\circ} \mathrm{F}\right)\end{array}$} & \multicolumn{2}{|c|}{ Strain } & \multirow{2}{*}{\multicolumn{2}{|c|}{ Life }} & \multirow[b]{2}{*}{$\begin{array}{l}\text { Stress } \\
\mathrm{MPa}\end{array}$} & \multirow[b]{2}{*}{$\begin{array}{l}\text { Range } \\
\text { ksi }\end{array}$} \\
\hline & $\begin{array}{c}\text { Range } \\
\%\end{array}$ & $\begin{array}{c}\text { Rate } \\
\mathrm{Hz}\end{array}$ & & & & \\
\hline \multicolumn{7}{|c|}{ Air Tests: As-received Condition } \\
\hline $850(1562)$ & $\begin{array}{l}0.3 \\
0.4 \\
0.7\end{array}$ & $\begin{array}{l}0.4 \\
0.4 \\
0.4\end{array}$ & $\begin{array}{r}734000 \\
3100 \\
850\end{array}$ & $\begin{array}{r}734000 \\
3685 \\
1250\end{array}$ & $\begin{array}{l}413.7 \\
572.3 \\
488.2\end{array}$ & $\begin{array}{l}60.0 \\
83.0 \\
70.8\end{array}$ \\
\hline
\end{tabular}

Simulated HTGR Helium Tests: As-received Condition

$\begin{array}{lllllll}850(1562) & 0.4 & 0.4 & 7800 & 11137 & 463.3 & 67.2 \\ 950(1742) & 0.4 & 0.4 & 5900 & 7153 & 391.6 & 56.8 \\ & 1.0 & 0.4 & 1000 & 1423 & 375.1 & 54.4\end{array}$

Simulated HTGR Helium Tests: Test Specimens Exposed 6000 Hours in Simulated HTGR Helium at Same Temperature as Test Temperature

$\begin{array}{lrrrrrr}850(1562) & 0.3 & 0.4 & 16300 & 18997 & 435.8 & 63.2 \\ & 0.4 & 0.4 & 5800 & 6363 & 463.3 & 67.2\end{array}$

$\begin{array}{llllll}0.6 & 0.4 & 1800 & 2246 & 554.3 & 80.4\end{array}$

$\begin{array}{lllllll}950(1742) & 0.4 & 0.4 & 7500 & 8156 & 386.1 & 56.0 \\ & 0.7 & 0.4 & 1400 & 1771 & 380.6 & 55.2\end{array}$

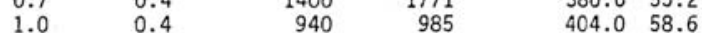

$\mathrm{a}=\mathrm{N}_{5}$ is the number of cycles to a $5 \%$ decrease in the stress range below the

săturation value.

$b=N_{f}$ is the number of cycles to a $50 \%$ decrease in stress range.

$c=$ Reported values are at $N_{f} / 2$.

\begin{tabular}{|l|l|l|l|}
\hline Data ID: & Baldwin486T10 & Data Class: & 4 \\
\hline Data Source: & GE HTGR Project & Reference: & Baldwin 1986 \\
\hline Comment: & \multicolumn{3}{|l|}{} \\
\hline
\end{tabular}

TABLE 10

INCONEL 617 FATIGUE TEST DATA (LCF), Heat No. XX63A8UK

\begin{tabular}{|c|c|c|c|c|c|}
\hline $\begin{array}{l}\text { Temperature } \\
{ }^{\circ} \mathrm{C}\left({ }^{\circ} \mathrm{F}\right)\end{array}$ & $\underset{\%}{\text { Strain Range }}$ & $\begin{array}{c}\text { Stra in Rate } \\
1 / \mathrm{Sec}\end{array}$ & $\underset{N_{5}}{\operatorname{Life}}$ & $\begin{array}{l}\text { Stre } \\
\mathrm{MPa}\end{array}$ & $\begin{array}{l}\text { S Range } \\
\text { ksi }\end{array}$ \\
\hline \multicolumn{6}{|c|}{ Air Tests: As-received Condition } \\
\hline $950(1742)$ & $\begin{array}{l}1.0 \\
0.6 \\
0.4 \\
0.3 \\
0.3 \\
0.3 \\
0.25\end{array}$ & $\begin{array}{l}0.4 \\
0.2 \\
0.1 \\
0.04 \\
0.05 \\
0.05 \\
0.05\end{array}$ & $\begin{array}{r}590 \\
1300 \\
2820 \\
22190 \\
9990 \\
6690 \\
19000\end{array}$ & $\begin{array}{l}441 \\
424 \\
412 \\
377 \\
365 \\
372 \\
368\end{array}$ & $\begin{array}{l}63.9 \\
61.5 \\
59.7 \\
54.7 \\
52.9 \\
53.9 \\
53.3\end{array}$ \\
\hline
\end{tabular}

Simulated HTGR Hel ium Tests: Test Specimens Exposed 6000 Hours in Simulated HTGR Helium at $950^{\circ} \mathrm{C}$

950 (1742)

1.0
1.0
0.6
0.6
0.4
0.3
0.25
0.25

0.4
0.05
0.05
0.15
0.1
0.05
0.05
0.05

$\begin{array}{rrr}1615 & 510 & 73.9 \\ 900 & 339 & 49.1 \\ 3200 & 359 & 52.1 \\ 2500 & 343 & 49.7 \\ 3500 & 336 & 48.7 \\ 11900 & 311 & 45.1 \\ 36000 & 359 & 52.1 \\ 47000 & 354 & 51.3\end{array}$

- - - -

$a=N_{5}$ is the number of cycles to a $5 \%$ decrease in the stress range below the

saturation value.

$b=$ Reported values are at approximately $N_{5} / 2$. 
ASSESSMENT OF EXISTING ALLOY 617 DATA FOR GEN IV NUCLEAR REACTOR APPLICATIONS

\begin{tabular}{|l|l|l|l|}
\hline Data ID: & Baldwin486T11 & Data Class: & 4 \\
\hline Data Source: & GE HTGR Project & Reference: & Baldwin 1986 \\
\hline Comment: &
\end{tabular}

TABLE 11

INCONEL 617 CREEP FATIGUE TEST DATA, Heat No. XX63A8UK

\begin{tabular}{ccccc} 
Temperature & Strain Range \\
${ }^{\circ} \mathrm{C}\left({ }^{\circ} \mathrm{F}\right)$ & $\begin{array}{c}\text { Strain Rate } \\
1 / \mathrm{Sec}\end{array}$ & $\begin{array}{c}\text { Life } \\
\mathrm{N}_{5}\end{array}$ & $\begin{array}{c}\text { Stress Range } \\
\mathrm{MPa}\end{array}$ \\
\hline
\end{tabular}

Air Tests: As-received Condition

950 (1742)

2 Minute Tensile Hold:

$\begin{array}{llrrr}1.0 & 0.05 & 420 & 352 & 51.1 \\ 0.6 & 0.1 & 850 & 372 & 53.9 \\ 0.4 & 0.08 & 2000 & 374 & 54.2 \\ 0.3 & 0.05 & 3100 & 352 & 51.1\end{array}$

20 Minute Tensile Hold:

$\begin{array}{llrrr}1.0 & 0.05 & 360 & 316 & 45.9 \\ 0.6 & 0.1 & 610 & 363 & 52.7 \\ 0.4 & 0.05 & 1750 & 314 & 45.5\end{array}$

- - - - -

$\mathrm{a}=\mathrm{N}_{5}$ is the number of cycles to a $5 \%$ decrease in the stress range below the saturation value.

$b=$ Reported values are at approximately $\mathrm{N}_{5} / 2$. 


\section{Huntington Alloys Data}

The Huntington Alloys data source is a collection of mechanical properties data on Alloy 617 provided by its manufacturer Huntington Alloys, Inc. Unlike the ORNLHTGR data and the GE-HTGR data that were generated for the purpose of design and construction of nuclear reactors, the Huntington Alloys data were produced for general applications of the alloy, which included manufacturing such components as ducting, combustion cans, transition liners in both aircraft and land-based gas turbines, catalystgrid supports for the production of nitric acid, heat-treating baskets in metallurgical processing, reduction boats in the refining of molybdenum and many others. The Huntington Alloys data collected for the present report include tensile properties data generated from 179 tensile tests at temperatures of 25, 38, 93, 149, 204, 260, 316, 371, $427,482,538,593,649,704,760,816,871,927,982,1038$, and $1093^{\circ} \mathrm{C}(75,100,200$, $300,400,500,600,700,800,900,1000,1100,1200,1300,1400,1500,1600,1700$, 1800,1900 , and $2000^{\circ} \mathrm{F}$ ), and creep properties data generated from 249 creep tests at temperatures of $593,649,704,760,816,871,927,982,1000,1038,1093^{\circ} \mathrm{C}(1100,1200$, $\left.1300,1400,1500,1600,1700,1800,1832,1900,2000^{\circ} \mathrm{F}\right)$. The tensile properties include yield strength, ultimate tensile strength, total strain, and reduction in area. The creep

Table 20: Summary of the Huntington Alloys data of Alloy 617

\begin{tabular}{|c|c|c|c|c|}
\hline $\begin{array}{c}\text { Temperature } \\
{ }^{\circ} \mathrm{C}\end{array}$ & $\begin{array}{c}\text { Tensile Tests } \\
\text { on Base Metal }\end{array}$ & $\begin{array}{c}\text { Tensile Tests } \\
\text { on Weld }\end{array}$ & $\begin{array}{c}\text { Creep Tests } \\
\text { on Base Metal }\end{array}$ & $\begin{array}{c}\text { Creep Tests } \\
\text { on Weld }\end{array}$ \\
\hline 24 & 26 & 9 & & \\
\hline 38 & 3 & & & \\
\hline 93 & 6 & 2 & & \\
\hline 149 & 3 & & & \\
\hline 204 & 6 & 2 & & \\
\hline 260 & 3 & & & \\
\hline 316 & 6 & 2 & & \\
\hline 371 & 3 & & & \\
\hline 427 & 6 & 2 & & \\
\hline 482 & 3 & & & \\
\hline 538 & 8 & 4 & & \\
\hline 593 & 5 & 2 & 7 & \\
\hline 649 & 9 & 4 & 22 & \\
\hline 704 & 4 & 2 & 8 & \\
\hline 760 & 9 & 4 & 24 & \\
\hline 816 & 3 & 3 & 28 & 3 \\
\hline 871 & 8 & 4 & 65 & 3 \\
\hline 927 & 3 & 2 & 6 & \\
\hline 982 & 6 & 4 & 29 & 2 \\
\hline 1000 & & & 16 & \\
\hline 1038 & 3 & & 5 & \\
\hline 1093 & 6 & 4 & 31 & \\
\hline Total & 129 & 50 & 241 & 8 \\
\hline
\end{tabular}


properties include creep rupture time, creep rupture stress, creep rupture strain, minimum creep rate, time to $1 \%$ total strain, and time to $0.2 \%$ offset tertiary creep. A summary of the tests that generated the Huntington Alloys data is given in Table 20. It should be pointed out that due to poor copy quality of the source documents obtained, some data are not included in this table. Efforts will continue to collect these missing data. Since the Huntington Alloys data came from the manufacturer, they should be classified as Class 5 . However, because these data have also been accepted for the development of several ASME B\&PV Code cases, such as the draft Code Case for the design of Alloy 617 nuclear components at the request of the DOE for the HTGR Program in the 1980's [Corum 1991], and applications in designing a natural gas reformer based on the requirements of ASME B\&PV Code Section VIII, Division 1 for producing synthesis gas, these data may be qualified as Class 2 after being reviewed and approved by the Gen IV Materials Handbook management.

Table 21: Compositions (wt. \%) of the Alloy 617 heats for the Huntington Alloys data

\begin{tabular}{|c|c|c|c|c|c|c|c|c|}
\hline Heat & Ni & Cr & Co & Mo & Fe & Mn & Al & C \\
\hline XX00A1US & 53.91 & 22.51 & 12.67 & 8.91 & 0.13 & 0.04 & 1.05 & 0.07 \\
\hline XX00A2US & 54.6 & 22.77 & 12.72 & 8.59 & 0.18 & 0.02 & 0.98 & 0.07 \\
\hline XX00A3US & 54.76 & 22.64 & 12.5 & 8.82 & 0.09 & 0.03 & 1.01 & 0.06 \\
\hline XX00A4US & 54.73 & 22.31 & 12.46 & 9.09 & 0.15 & 0.02 & 1.06 & 0.07 \\
\hline XX00A5US & 55.91 & 21.77 & 12.24 & 8.71 & 0.19 & 0.03 & 0.99 & 0.07 \\
\hline XX05A4UK & 54.97 & 22.04 & 12.46 & 9 & 0.24 & 0.02 & 1.08 & 0.07 \\
\hline XX05A7UK & 55.02 & 21.77 & 12.57 & 9.15 & 0.21 & 0.01 & 1.07 & 0.06 \\
\hline XX07A7UK & 55.12 & 21.99 & 12.3 & 8.52 & 0.52 & 0.02 & 1.31 & 0.08 \\
\hline XX10A3UK & 54.16 & 22.17 & 12.7 & 9.29 & 0.33 & 0.04 & 1.04 & 0.09 \\
\hline XX18A4UK & 54.22 & 21.86 & 12.35 & 8.95 & 0.72 & 0.01 & 1.03 & 0.051 \\
\hline XX20A5UK & 55.61 & 21.32 & 12.67 & 8.85 & 0.28 & 0.01 & 1.05 & 0.06 \\
\hline XX26A8UG & 54.54 & 21.89 & 12.48 & 9 & 0.48 & 0.03 & 1.17 & 0.06 \\
\hline XX41A7UK & 54.01 & 21.42 & 12.9 & 8.83 & 1.35 & 0.02 & 0.92 & 0.06 \\
\hline Heat & $\mathbf{C u}$ & $\mathbf{S i}$ & $\mathbf{S}$ & $\mathbf{T i}$ & $\mathbf{P}$ & $\mathbf{B}$ & $\mathbf{N}$ & $\mathbf{M g}$ \\
\hline XX00A1US & 0.23 & 0.04 & 0.007 & 0.41 & 0.003 & 0.0051 & & 0.029 \\
\hline XX00A2US & 0.01 & 0.04 & 0.008 & 0.25 & 0.002 & 0.005 & 0.019 & 0.022 \\
\hline XX00A3US & 0.01 & 0.06 & 0.007 & 0.39 & 0.004 & 0.004 & 0.023 & 0.007 \\
\hline XX00A4US & 0.01 & 0.08 & 0.007 & 0.35 & 0.003 & 0.0043 & 0.012 & 0.029 \\
\hline XX00A5US & 0.02 & 0.06 & 0.007 & 0.46 & 0.003 & 0.0059 & 0.011 & 0.024 \\
\hline XX05A4UK & 0.07 & 0.12 & 0.002 & 0.43 & 0.006 & 0.002 & & 0.043 \\
\hline XX05A7UK & 0.07 & 0.14 & 0.004 & 0.51 & 0.004 & 0.002 & & 0.051 \\
\hline XX07A7UK & 0.09 & 0.14 & 0.003 & 0.43 & 0.004 & 0.001 & & 0.07 \\
\hline XX10A3UK & 0.19 & 0.18 & 0.003 & 0.32 & 0.003 & 0.001 & & 0.04 \\
\hline XX18A4UK & 0.09 & 0.17 & 0.006 & 0.28 & 0.001 & 0.003 & & 0.03 \\
\hline XX20A5UK & 0.1 & 0.15 & 0.001 & 0.27 & 0.002 & 0.002 & & 0.021 \\
\hline XX26A8UG & & 0.09 & 0.001 & 0.26 & 0.001 & 0.002 & & 0.039 \\
\hline XX41A7UK & 0.04 & 0.19 & 0.001 & 0.3 & 0.002 & 0.001 & & 0.051 \\
\hline
\end{tabular}

The Huntington Alloys data that are reviewed in the present assessment were generated from thirteen heats plus one heat for welding filler metal. Chemical compositions and product forms of the thirteen heats are presented in Tables 21 and 22, 
respectively. All the heats were solution annealed. The welds were produced using Alloy 617 welding electrodes of four different diameters. The chemical compositions of the electrodes are given in Table 23. The welds were produced using tungsten inert gas (TIG) welding and pulsed gas metal arc welding processes.

Table 22: Product forms of the Alloy 617 for the Huntington Alloys data

\begin{tabular}{|c|c|c|c|}
\hline Heat & Product Form & Heat & Product Form \\
\hline \multirow{4}{*}{ XX00A1USL } & 0.062" Cold Rolled Sheet & \multirow{2}{*}{ XX00A4USL } & 1" Hot Rolled Round \\
\hline & 3/4" Hot Rolled Round & & Hot Rolled Round \\
\hline & Cold Rolled Sheet & \multirow{2}{*}{ XX05A4UK } & 1/2" Hot Rolled Round \\
\hline & Hot Rolled Round & & Hot Rolled Round \\
\hline \multirow{3}{*}{ XX00A2USL } & 3/4" Hot Rolled Round & \multirow{2}{*}{ XX05A7UK } & 1" CD Tube \\
\hline & 9/16" Hot Rolled Round & & CD Tube \\
\hline & Hot Rolled Round & \multirow{2}{*}{ XX07A7UK } & 5/8" Hot Rolled Round \\
\hline \multirow{5}{*}{ XX00A3USL } & 1" Flat & & Hot Rolled Round \\
\hline & 1/2" Flat & XX10A3UK & Extruded Tube \\
\hline & 12" Square & \multirow{2}{*}{ XX18A4UK } & $0.188 "$ Cold Rolled Sheet \\
\hline & Forged Square & & Cold Rolled Sheet \\
\hline & Hot Rolled Flat & \multirow{2}{*}{ XX20A5UK } & 0.187" Cold Rolled Sheet \\
\hline \multirow{4}{*}{ XX00A5USL } & 0.032" Cold Rolled Sheet & & Cold Rolled Sheet \\
\hline & 5/8" Hot Rolled Round & XX26A8UK & 0.040" Cold Rolled Sheet \\
\hline & Cold Rolled Sheet & \multirow{2}{*}{ XX41A7UK } & Cold Rolled Sheet \\
\hline & Hot Rolled Round & & \\
\hline
\end{tabular}

Note: For heats that have same product form with and without a dimension prefix, the product form descriptions are original from the source. They may have the same dimension.

Table 23: Chemical compositions of Alloy 617 welding electrodes used for generating the Huntington Alloys data

\begin{tabular}{|l|c|c|c|c|c|c|c|c|c|c|c|c|c|}
\hline \multicolumn{1}{|l|}{ Diameter } & \multicolumn{10}{|c|}{ Element } \\
\hline mm & inch & Ni & Cr & Co & Mo & Fe & Mn & Al & C & Si & S & Ti & Nb+Ta \\
\hline 2.38 & $3 / 32$ & Bal & 23.07 & 11.48 & 9.24 & 1.15 & 1.45 & 0.22 & 0.10 & 0.50 & 0.005 & 0.09 & 0.56 \\
\hline 3.18 & $1 / 8$ & Bal & 23.14 & 11.47 & 9.23 & 1.18 & 1.39 & 0.21 & 0.11 & 0.49 & 0.004 & 0.08 & 0.47 \\
\hline 3.97 & $5 / 32$ & Bal & 23.33 & 11.71 & 9.32 & 0.89 & 0.66 & 0.26 & 0.10 & 0.48 & 0.004 & 0.10 & 0.02 \\
\hline 4.76 & $3 / 16$ & Bal & 23.16 & 11.75 & 9.33 & 0.85 & 0.63 & 0.22 & 0.10 & 0.46 & 0.003 & 0.16 & N/A \\
\hline
\end{tabular}


ASSESSMENT OF EXISTING ALLOY 617 DATA

FOR GEN IV NUCLEAR REACTOR APPLICATIONS

ORIGINAL HUNTINGTON ALLOYS DATA 
Data ID:

HA83T1

Data Source: Huntington Alloys Comment:
Data Class: 5

Reference: Huntington 1983

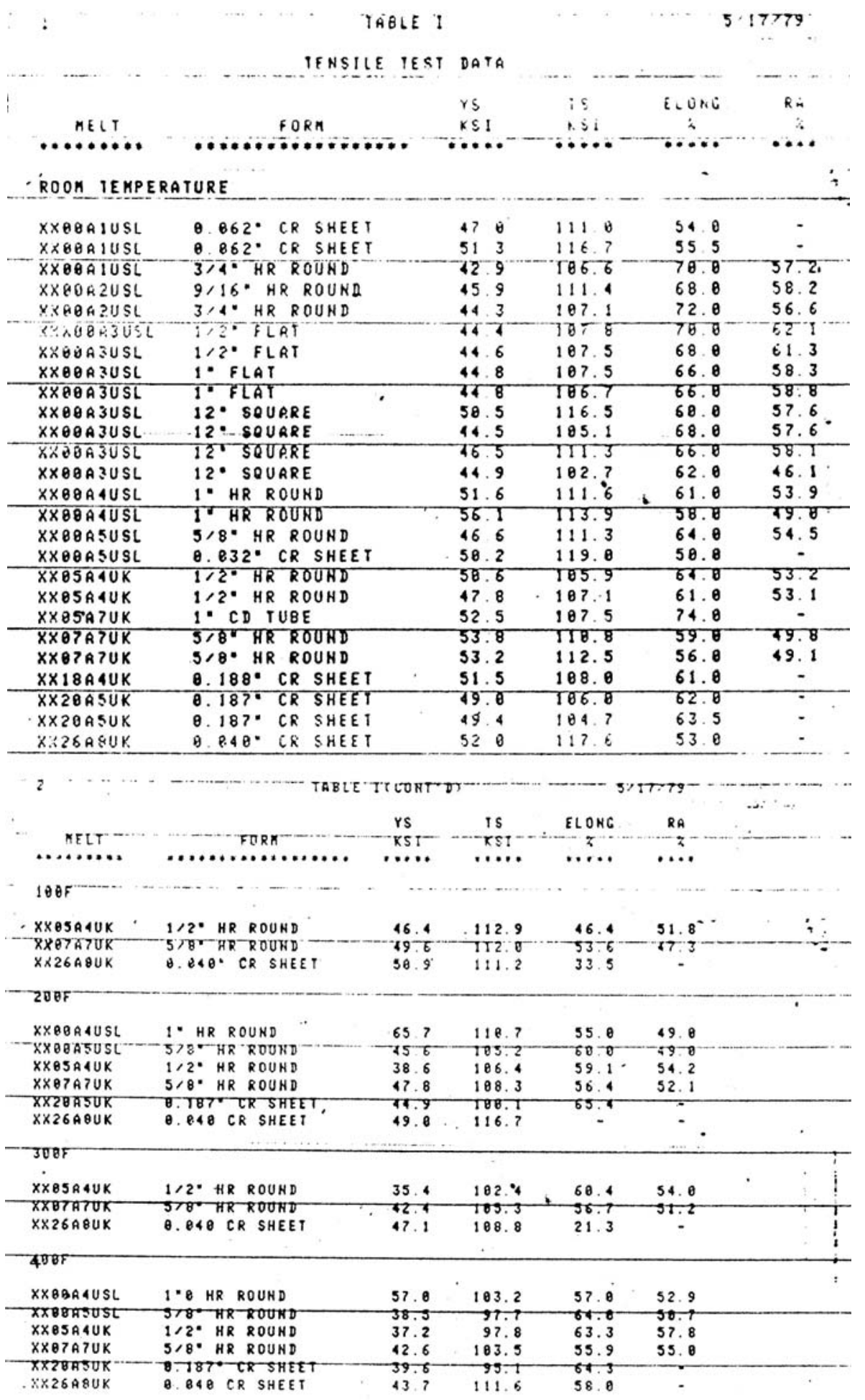




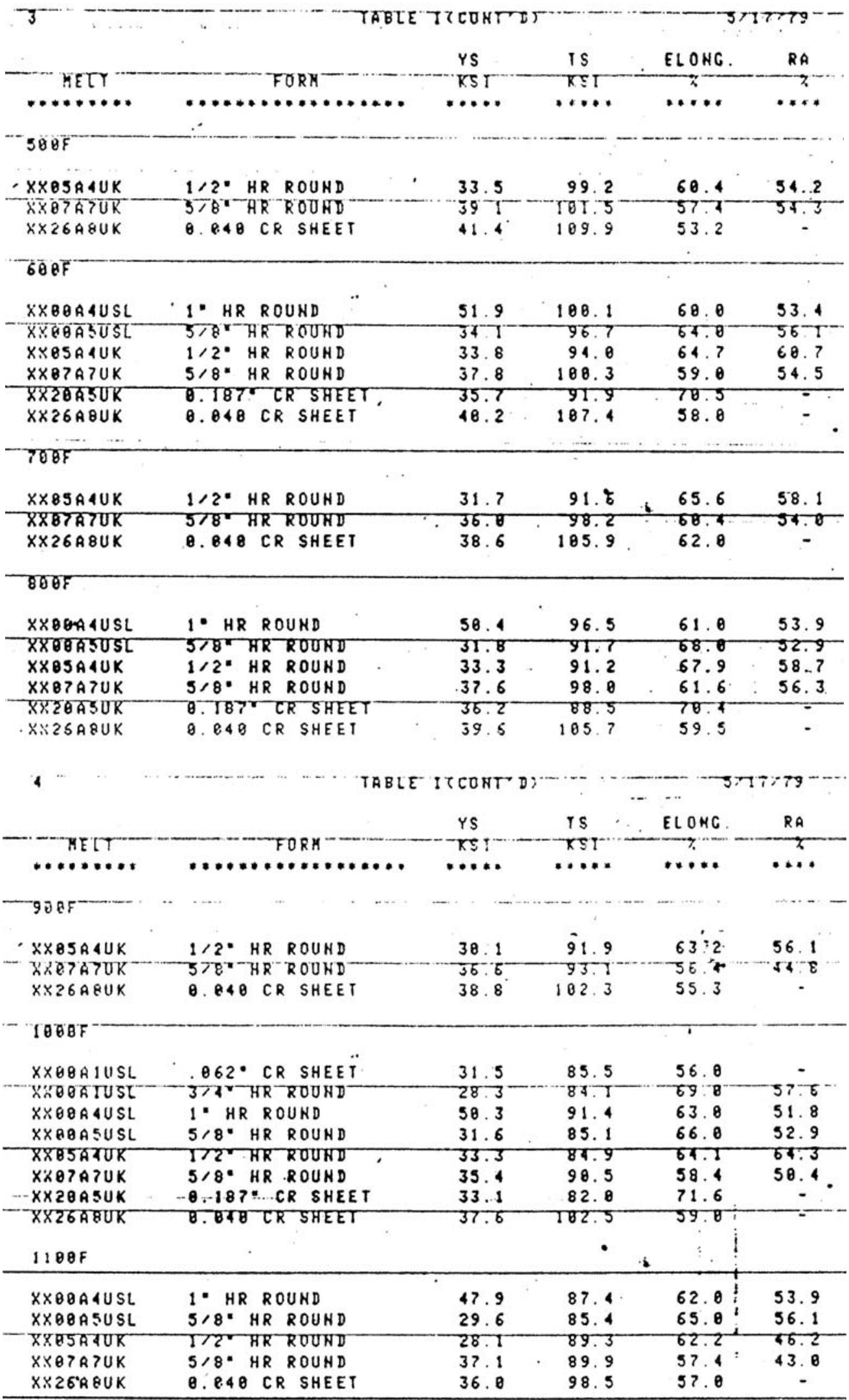




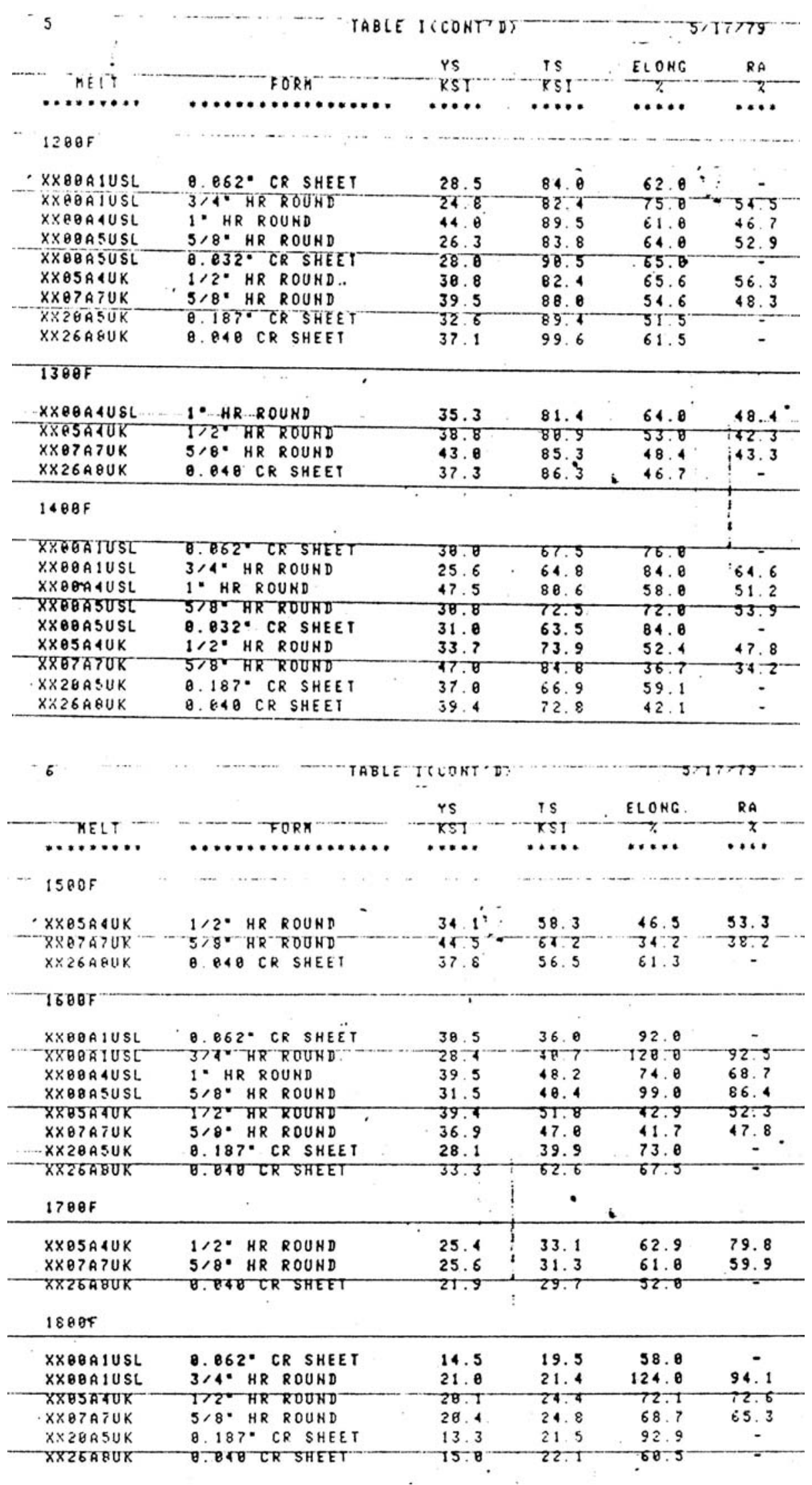


ASSESSMENT OF EXISTING ALLOY 617 DATA FOR GEN IV NUCLEAR REACTOR APPLICATIONS

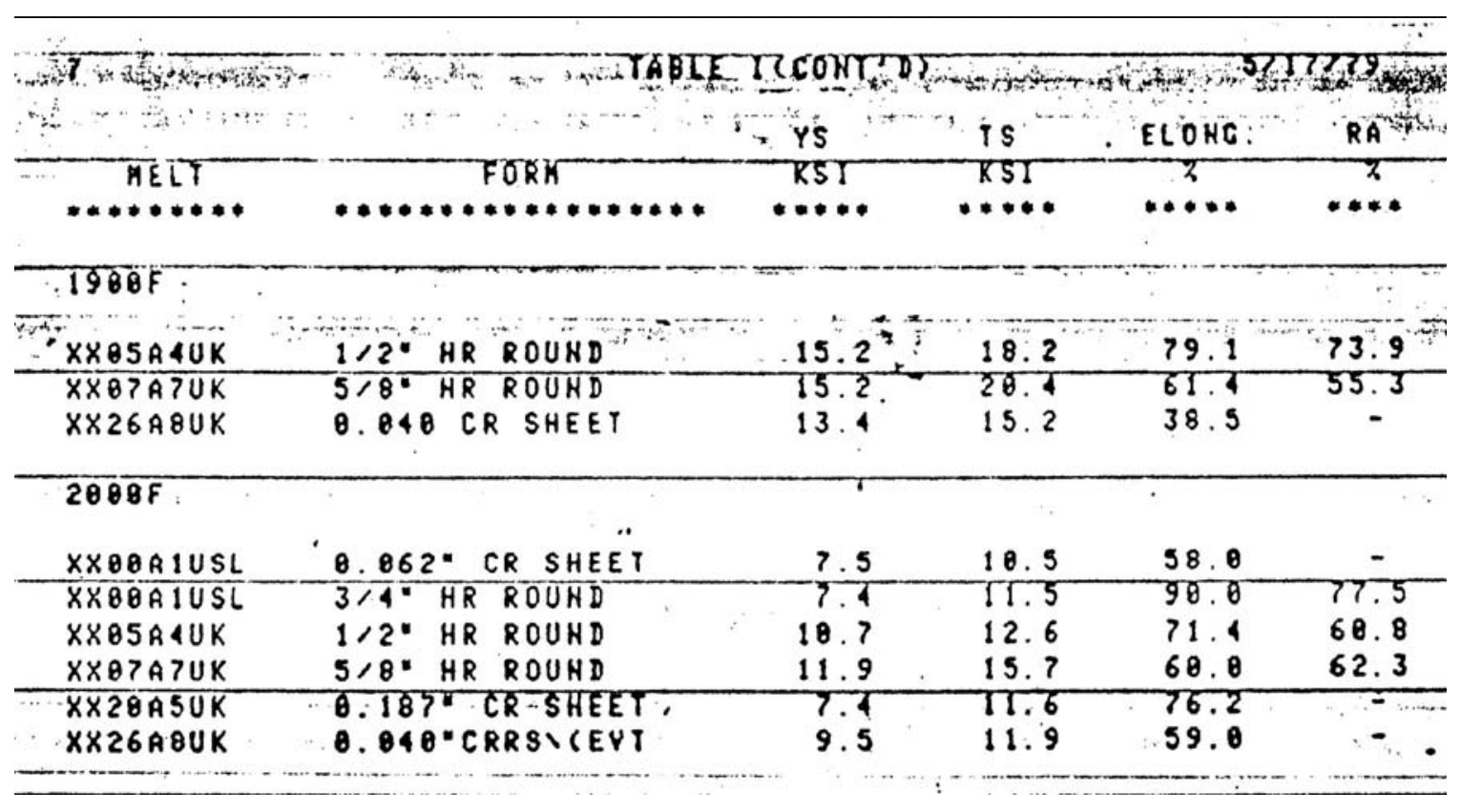


Data ID:

Data Source: Huntington Alloys Comment:
Data Class: 5

Reference: Huntington 1983

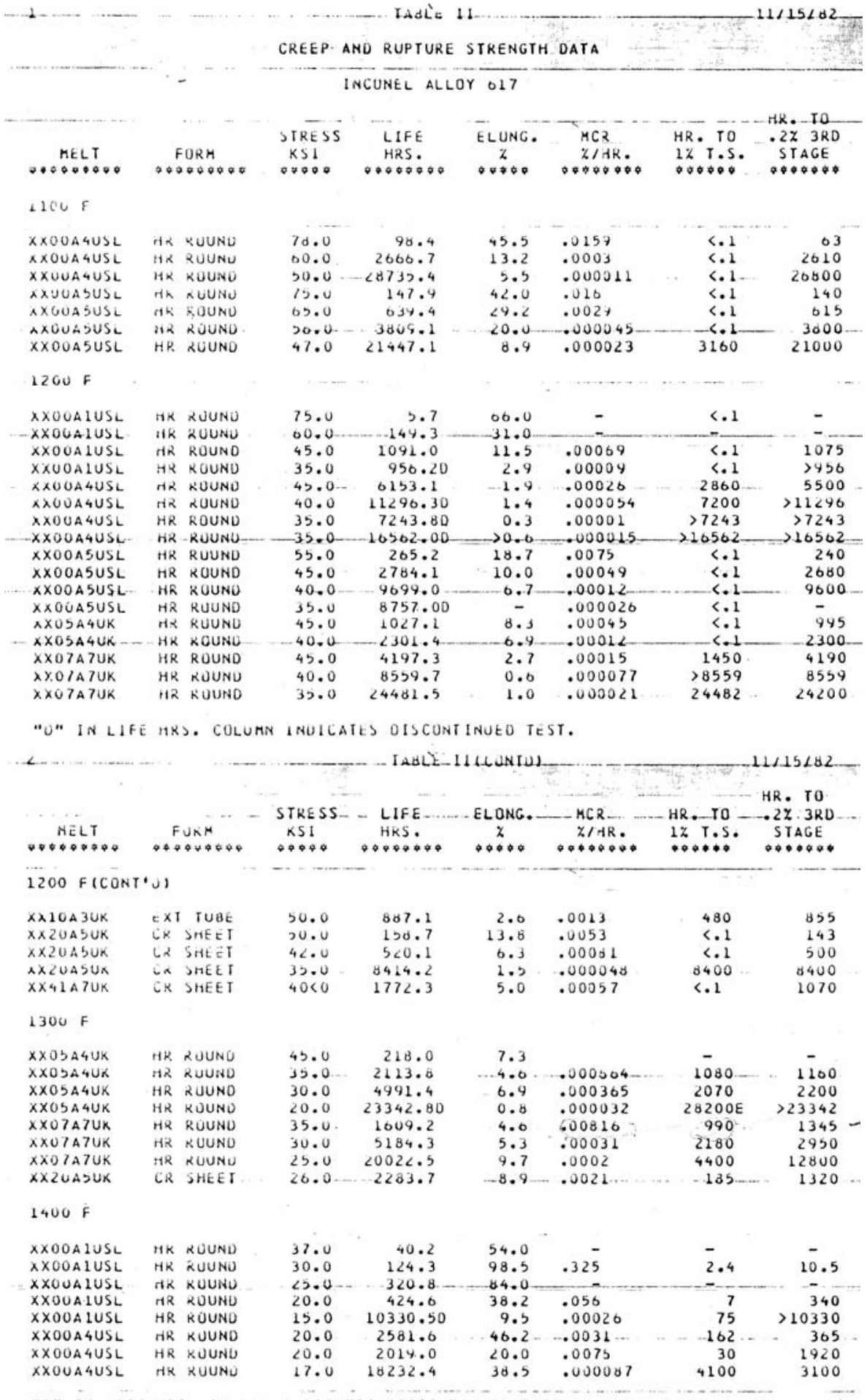

"O" in life hrs. COLUMn indicates discontinued test. 


\section{ASSESSMENT OF EXISTING ALLOY 617 DATA FOR GEN IV NUCLEAR REACTOR APPLICATIONS}

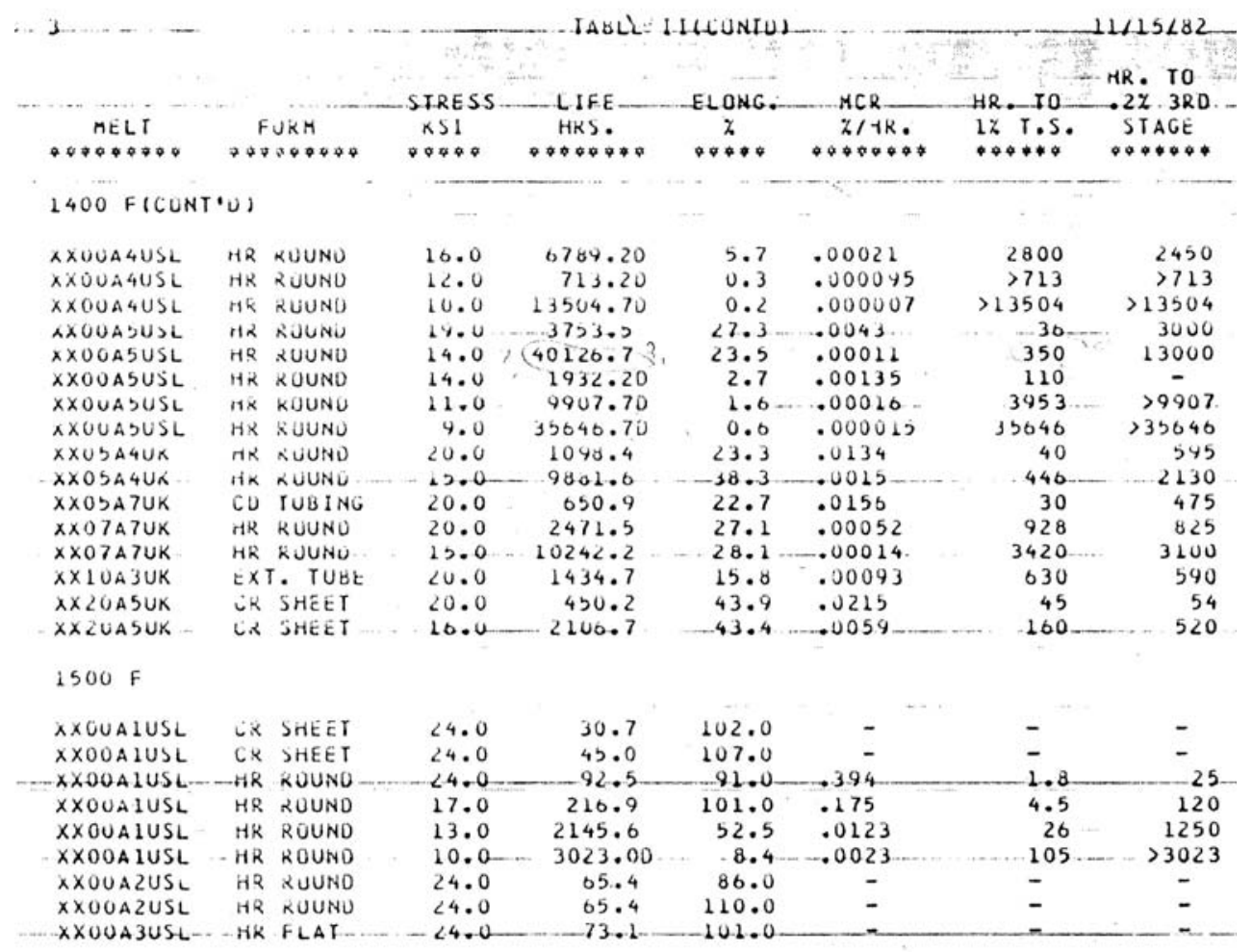

"D" In LifE hiRs. CULUMn indicates discontinueo test.

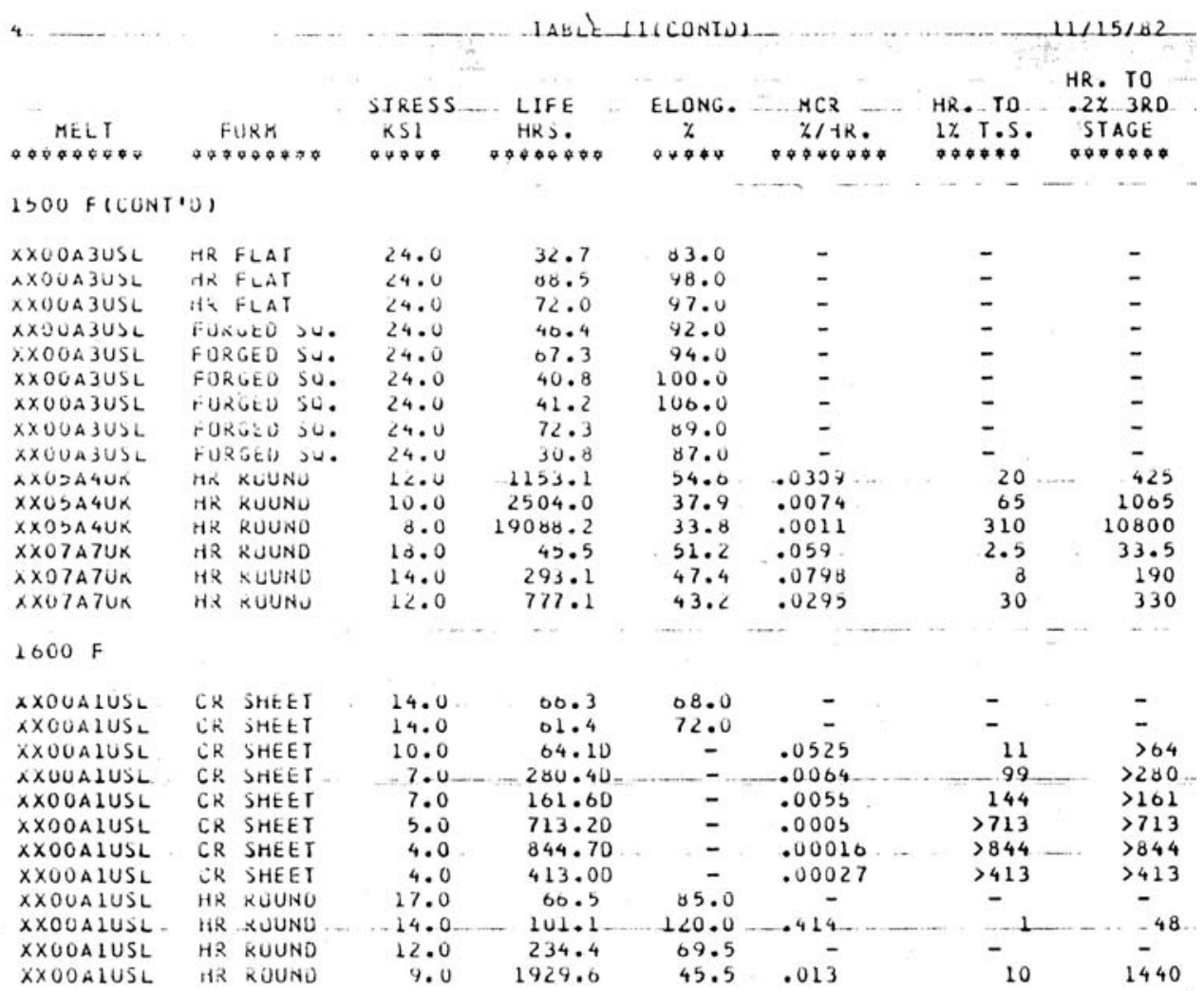

"D" in lif aks. Culumir inulcates olscontinued test. 


\section{ASSESSMENT OF EXISTING ALLOY 617 DATA FOR GEN IV NUCLEAR REACTOR APPLICATIONS}

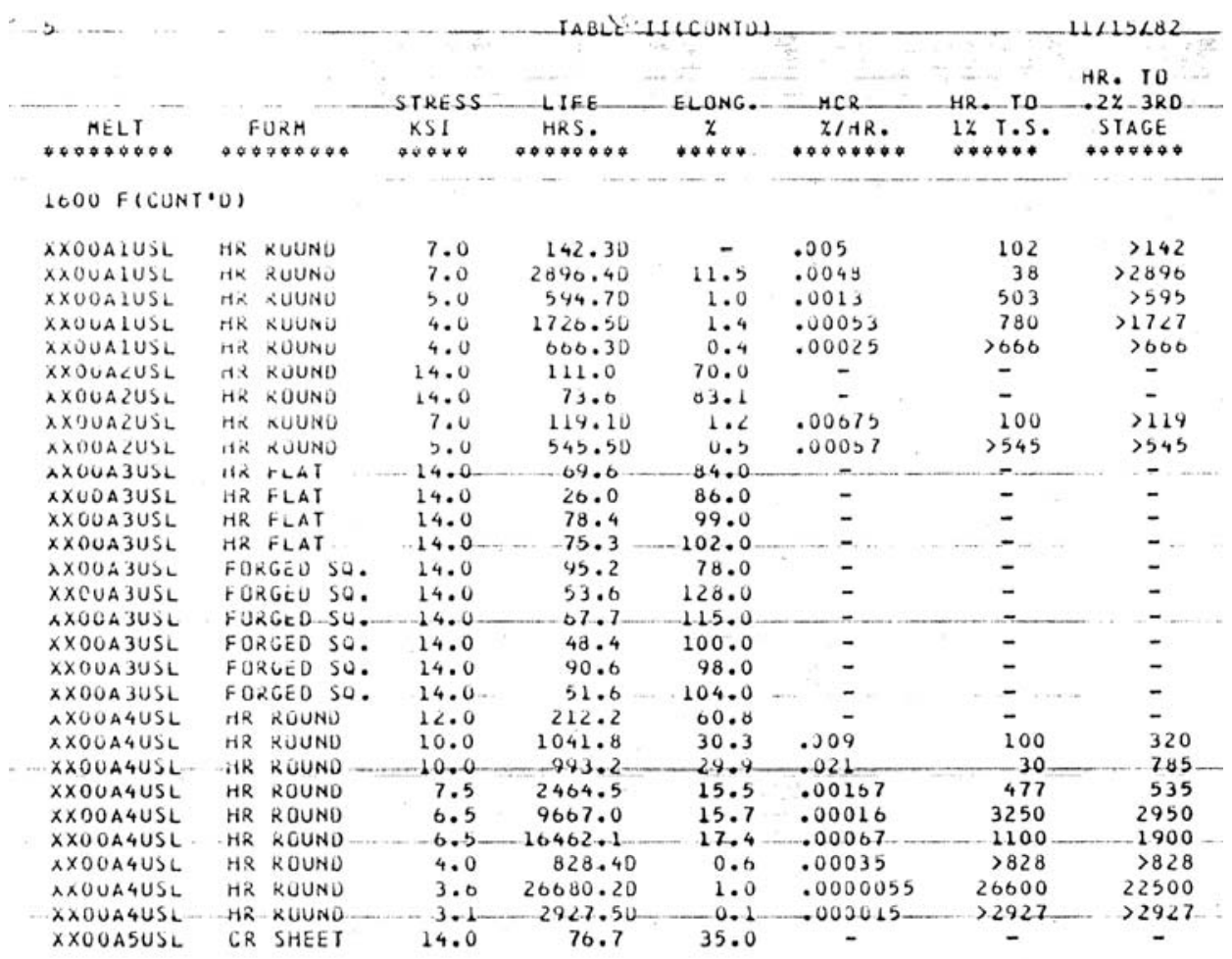

"D" in life hrs. CUlumin INDICATES oiscontinued test.

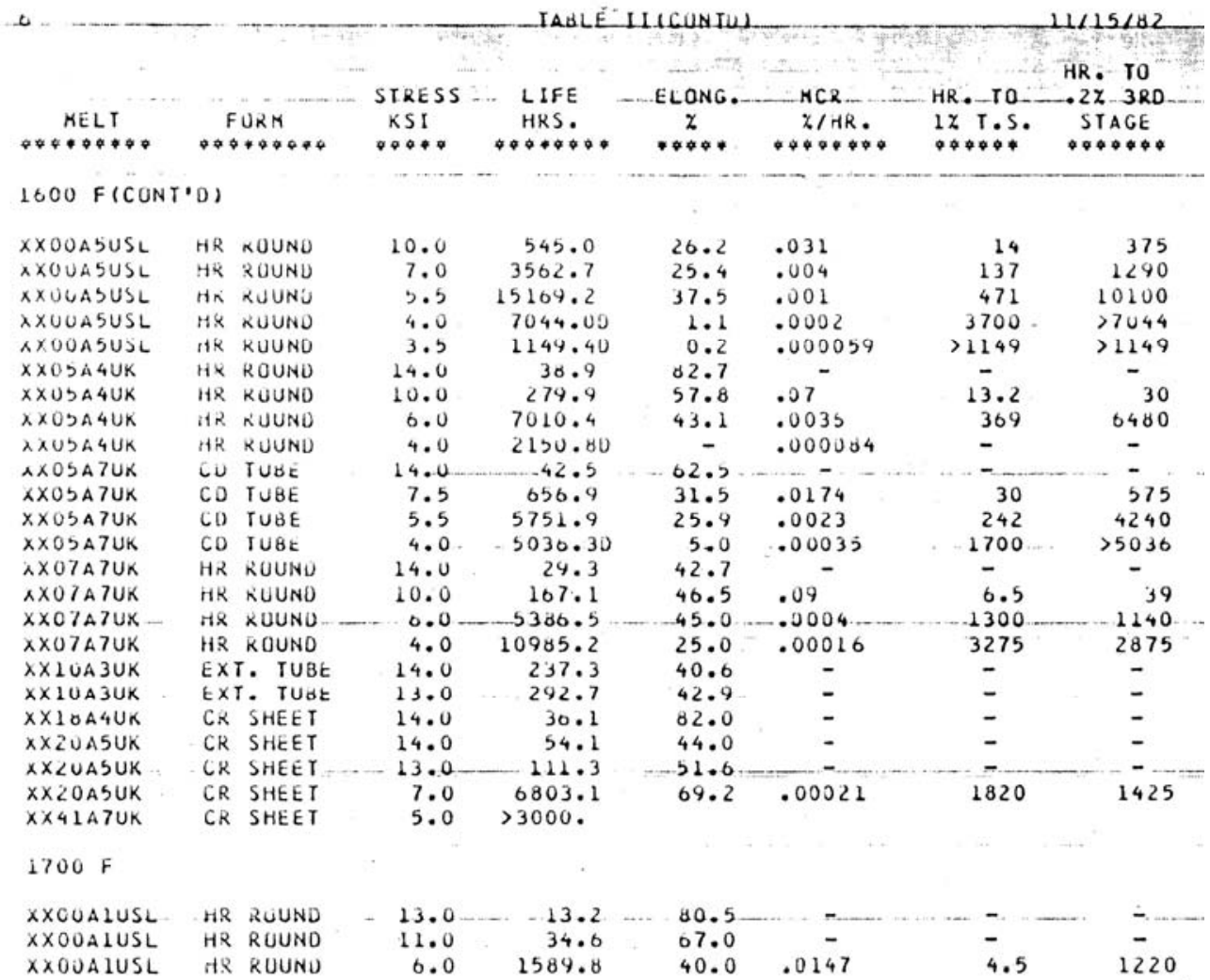

"U" in life hrs. COLUMi indicates discuntinued test. 


\section{ASSESSMENT OF EXISTING ALLOY 617 DATA FOR GEN IV NUCLEAR REACTOR APPLICATIONS}

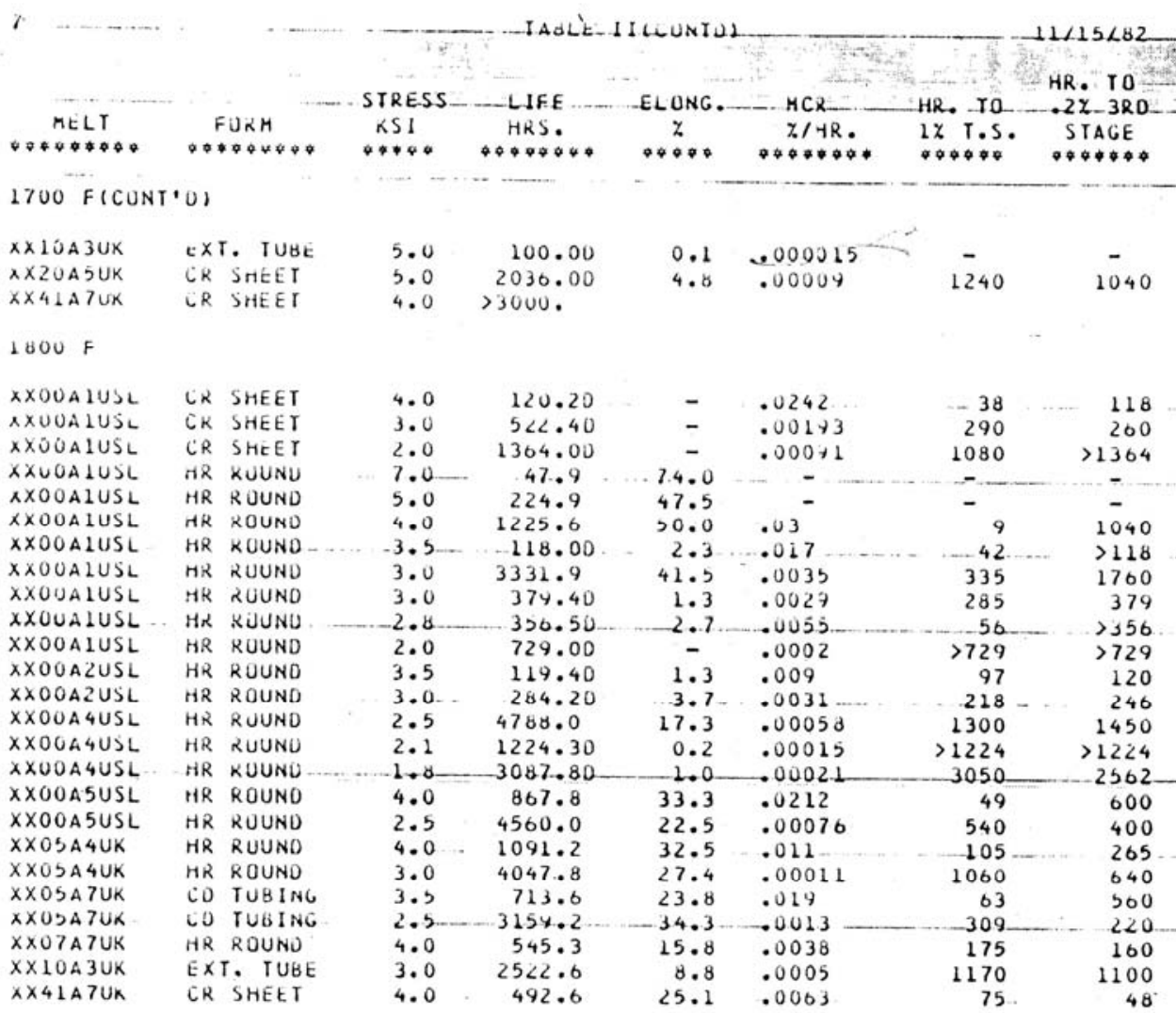

"1)" IN LIFE hRS. CULUMn INDICATLS DISCONTINUEU test.

\begin{tabular}{|c|c|c|c|c|c|c|c|c|}
\hline$\ldots$ & & & - & : & & 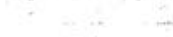 & HR. & . ro \\
\hline$\ldots$ & & & STRESS & LIFE & ELONG. & $\therefore$ MCR . & $\mathrm{HR}$. TO & $Z 3 R D$ \\
\hline MELT & & FURM & KSI & HKS. & $z$ & Wh & $1 \%$ T.S. & TAGE \\
\hline 000004000 & +40 & 4000404 & +0409 & $\phi \phi \$ \phi \phi \phi+\phi$ & 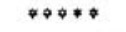 & $\$ 004040 *$ & 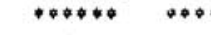 & 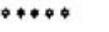 \\
\hline $1832 \mathrm{~F}$ & & & $\cdots-$ & . & & $\cdots \cdots \cdots-$ & & ...... \\
\hline XXOUAZUSL & $H R$ & RUUND & 2.0 & 104.0 & 58.4 & - & - & - \\
\hline XXOUAZUSL & $H R$ & ZUUND & 5.0 & 137.3 & 53.2 & - & - & - \\
\hline XXOOAZUSL & HR & KUUND & 4.5 & 353.0 & 42.2 & - & - & - \\
\hline XXOUAGUSL & $\mathrm{HK}$ & RUUND & 4.0 & 749.8 & 31.1 & .0022 & $263 \ldots$ & 240 \\
\hline XXOOA & MR & RUUNO & 2.9 & 1263.80 & - & .00215 & $331(7,1)$ & 640 \\
\hline XX0OA4USL & $H R$ & ROUND & 1.3 & 1340.50 & - & .000173 & $>1340$ & $>1340$ \\
\hline XXOUASUSL & HK & KUUNO & 4.0 & 444.5 & 38.0 & - & - & - \\
\hline XXOOASUSL & itR & RUUNO & 4.0 & 60.1 & 31.4 & - & - & - \\
\hline XXOUASUSL & $H X$ & KUUNO & 1.5 & 1816.40 & - & .00012 & $>1816$ & $>1016$ \\
\hline$X \times O>A 4 U K$ & HR & RUUNU & 5.0 & 156.9 & 17.6 & $\ldots \ldots$ & - & - \\
\hline$X \times 05 A 4 U K$ & $H R$ & ROUND & 3.0 & 1975.4 & 23.6 & .00064 & 543 & 400 \\
\hline$X X 0 S A 4 U K$ & $H R$ & ROUND & 1.5 & 934.30 & - & .00003 & - & - \\
\hline XX05A7UK & $\mathrm{CD}$ & TUBE & 5.0 & 157.4 & 37.4 & - & - & - \\
\hline XXUSA7UK & $C D$ & TUBE & $\angle .5$ & 1337.9 & 18.8 & .00375 & 182 & 217 \\
\hline$x \times 07 A 7 U K$ & $H K$ & RUUNO & 5.0 & 94.8 & 19.3 & - & - & - \\
\hline$\times \times 07 A 7 U K$ & $H R$ & ROUND & 1.5 & 1308.40 & $\ldots$ & .0002 & $=-$ & - \\
\hline $1900 \mathrm{~F}$ & & & & & & & & \\
\hline$\times \times 00 A 1 U S L$ & $H R$ & KUUND & 6.0 & 20.3 & 06.0 & - & - & - \\
\hline XXOOAIUSL & $H R$ & ROUND & 4.0 & 157.1 & 54.2 & .156 & 4.5 & 115 \\
\hline XXOOAIUSL & $H R$ & KUUND & 3.6 & 242.1 & $44.3 \ldots$ & $\ldots-$ & $-\ldots$ & - \\
\hline XXOUAIUSL & $H R$ & ROUND & 2.4 & 1165.1 & 26.5 & .013 & 43 & 735 \\
\hline XXOOAIUSL & $H R$ & KOUND & 2.0 & 2621.4 & 41.0 & .00021 & 320 & 160 \\
\hline $2000 \mathrm{~F}$ & & & & . & & & & \\
\hline XX00A1USL & $\mathrm{CR}$ & SHEET. & -3.0 & 41.1. & $-40.0 \ldots$ & - & $\therefore-$ & - \\
\hline XXOUALUSL & $C R$ & SHEET & 3.0 & 49.3 & 42.0 & - & - & - \\
\hline$X X O O A I U S L$ & $C R$ & SHEET & 1.5 & 137.50 & - & .0045 & 80 & 35. \\
\hline
\end{tabular}


ASSESSMENT OF EXISTING ALLOY 617 DATA FOR GEN IV NUCLEAR REACTOR APPLICATIONS

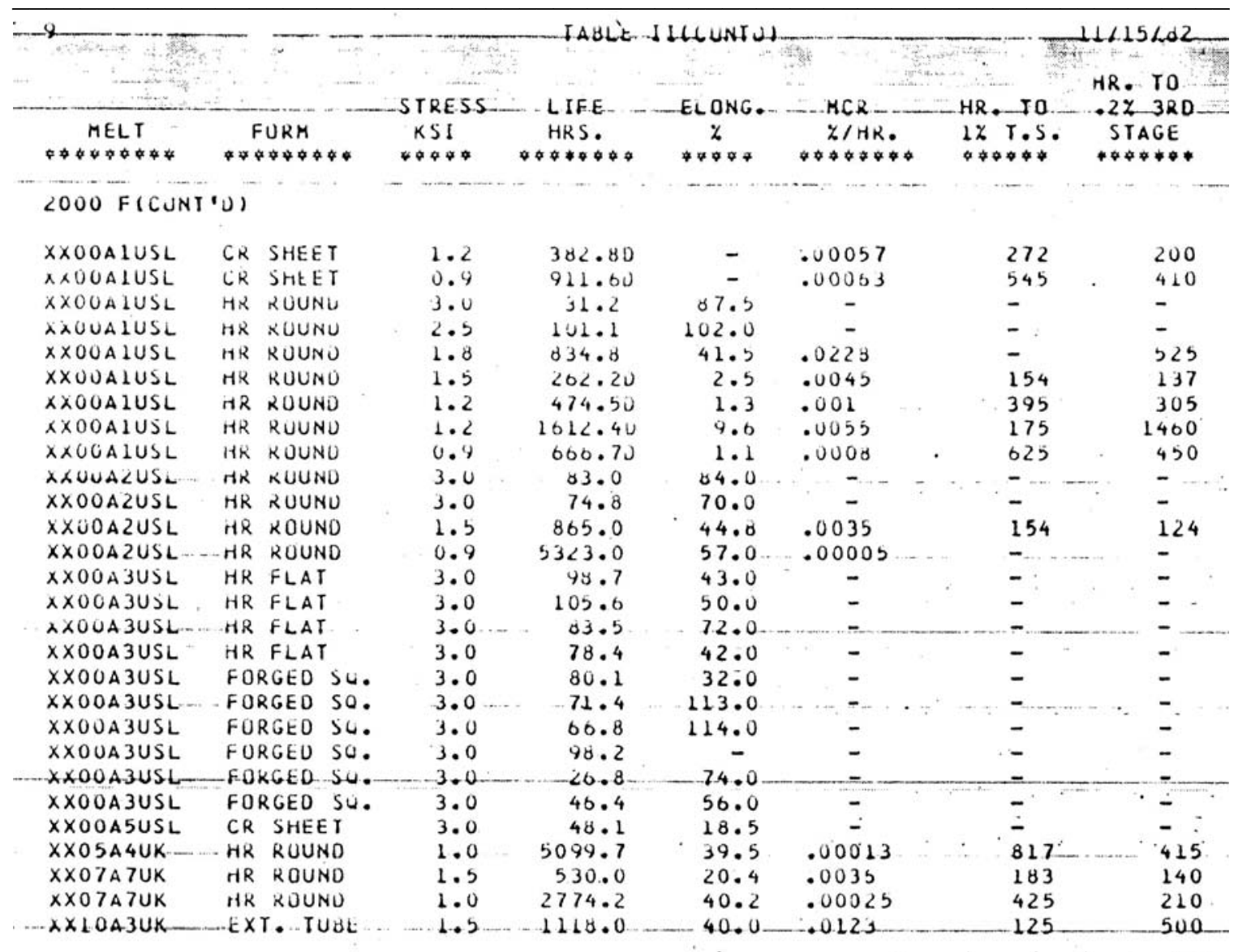

"D" in life hrs. Columir indicates oiscontinued test. 


\section{German HTGR Data}

When the U. S. was generating data on Alloy 617 for the HTGR project during the 1970's and 1980's, the Germans also selected Alloy 617 as one of the candidate materials for their nuclear reactor program and produced a large amount of data. The source document for the present assessment is a published summary paper [Schubert 1984] containing experimental results of several candidate materials including Alloy 617 generated in the 1980's in the German HTGR Materials Test Program. These experiments included tests up to approximately 20000 hours. The goal of the test program was to generate long term creep rupture data in the temperature range of 800 to $1000^{\circ} \mathrm{C}\left(1472\right.$ to $\left.1932^{\circ} \mathrm{F}\right)$ to enable extrapolation to 100000 hours of creep rupture life. The effects of the HTGR service environments on the mechanical behavior of the material were also investigated. Because it is a published journal paper, no tabulated materials mechanical data are provided. All the mechanical properties data are presented in plots. If acquisition of the original numerical data, which is currently in negotiation, can not be successfully achieved, digitization of the plots will be needed to prepare data for the input into the Gen IV Materials Handbook; and certain tolerance must be allowed for digitization error. Also, because no tabulated mechanical properties data are presented in the paper, the total number of tests conducted to generate the data can only be estimated by counting data points in the figures exhibited. When different numbers of data points are presented for different creep properties at a same given temperature, the greater number is considered to be closer to the actual number of the tests. For example, at $800^{\circ} \mathrm{C}, 24$ data points were presented for creep rupture stress while 45 data points for time to $1 \%$ creep strain. The larger number 45 is considered to be the test number based on the assumption that the difference in 24 and 45 may have resulted from termination of some tests before rupture occurred, therefore, more 1\% creep strain data points were obtained than creep rupture data points. In some figures where data points severely overlap, the number of tests can only be approximately estimated. Counting data points in the figures in such a manner indicates that the paper includes creep properties data generated from approximately 294 creep tests at temperatures of $800,850,900,950$, and $1000^{\circ} \mathrm{C}\left(1472,1562,1652,1742\right.$, and $\left.1832^{\circ} \mathrm{F}\right)$. The resulting summary of the tests that generated the German HTGR data is given in Table 24. The creep properties include time to $1 \%$ total strain, creep rupture time, creep rupture stress, creep strain, and minimum creep rate. Based on the Gen IV Materials Handbook Data Classification criteria, the data are classified as Class 5.

Table 24: Summary of the assessed German HTGR data of Alloy 617

\begin{tabular}{|c|c|c|c|c|c|c|}
\hline Specimen & Test & \multicolumn{5}{|c|}{ Test Temperature $^{\mathbf{0}} \mathbf{C}$} \\
\hline Type & Environment & $\mathbf{8 0 0}$ & $\mathbf{8 5 0}$ & $\mathbf{9 0 0}$ & $\mathbf{9 5 0}$ & $\mathbf{1 0 0 0}$ \\
\hline Base Metal & Air & 45 & 78 & 55 & 62 & 29 \\
\hline \multirow{2}{*}{$\begin{array}{c}\text { Weld with Matching } \\
\text { Filler Metal }\end{array}$} & Air & & 2 & & & \\
\cline { 2 - 7 } & He & & 2 & & & \\
\cline { 2 - 7 } & Processing Gas* & & 9 & & & \\
\hline Weld with & Air & & 4 & & & \\
\hline & He & & 4 & & 9 & \\
\hline
\end{tabular}

* methane reforming gas 
The German HTGR data were generated from several heats of Alloy 617 provided by Huntington Alloys and VDM. All these heats were from commercial or semicommercial production. No chemical compositions of the heats were presented in the paper. It was stated in the paper that no attempt was made to obtain special heats that would allow systematic investigations of compositional variations within the specification or of grain size effects. The material was solution treated at temperatures 1150 to $1205^{\circ} \mathrm{C}$ for 0.1 to 0.5 hour depending on grain sizes and then cooled in air.

The weld specimens for the German HTGR data exhibited in Table 24 were all transverse weldment specimens consisting of weld metal in the center gage section and base metal on both sides. The "Filler Metal 112" specimens were machined from weldment produced using the Inconel welding electrode 112 (Ni-22Cr-9Mo-3.5Nb). No post welding treatment was reported.

Creep strain of the German HTGR data were measured in two fashions. To maximize the number of specimens simultaneously being tested for long-term creep properties, multi-specimen creep rigs were used. The creep strains of specimens tested in the multispecimen creep rigs were measured at intervals by interruption of the test. Only those tested in single specimen creep rigs were measured for strain by continuous monitoring. 
ASSESSMENT OF EXISTING ALLOY 617 DATA

FOR GEN IV NUCLEAR REACTOR APPLICATIONS

ORIGINAL GERMAN HTGR DATA 
ASSESSMENT OF EXISTING ALLOY 617 DATA

FOR GEN IV NUCLEAR REACTOR APPLICATIONS

\begin{tabular}{|l|l|l|l|}
\hline Data ID: & Schubert84F1 & Data Class: & 5 \\
\hline Data Source: & German HTGR Project & Reference: & Schubert 1984 \\
\hline Comment: Creep in air &
\end{tabular}
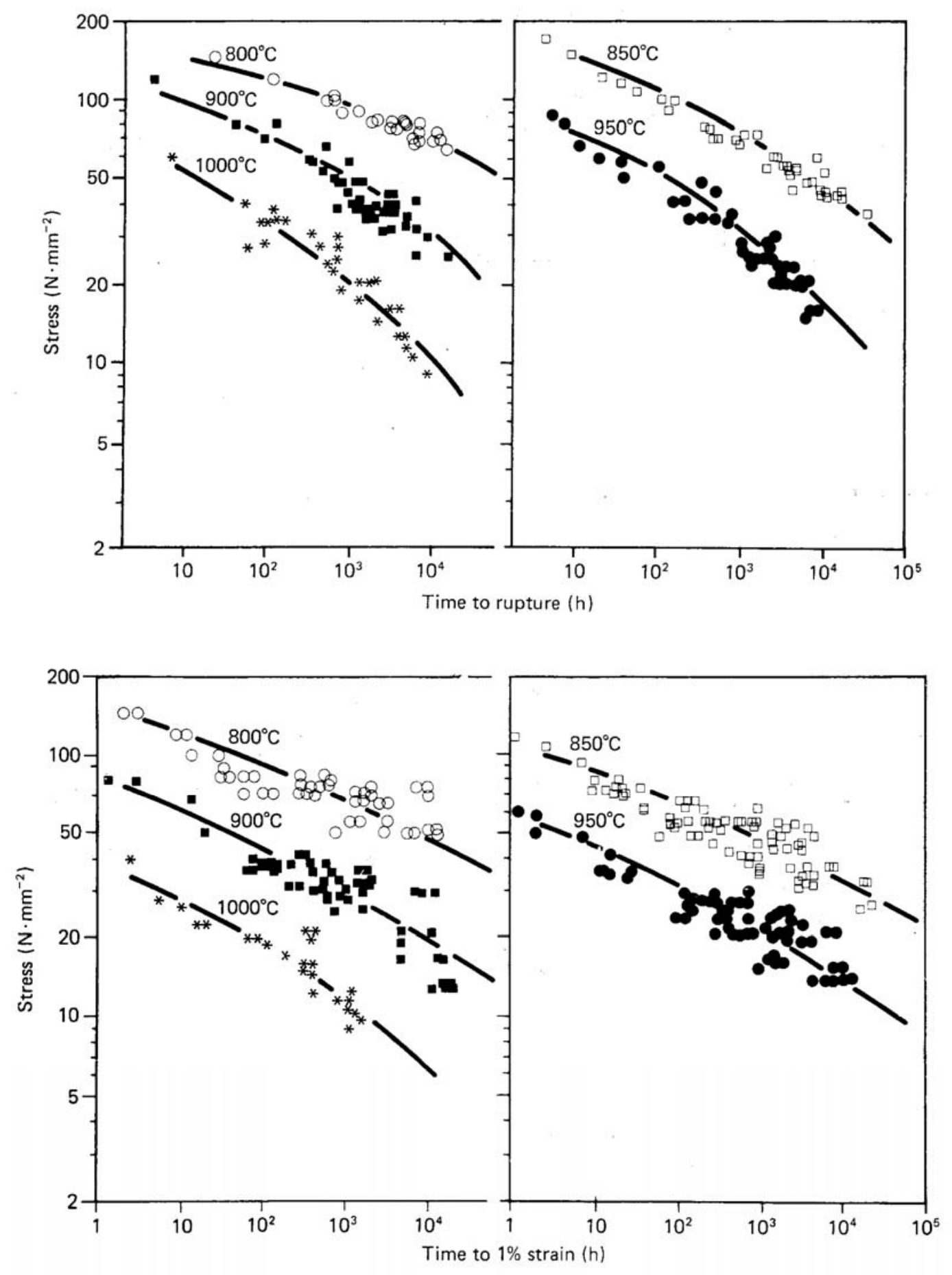

Fig. 1. Creep rupture properties of Inconel-617. 
ASSESSMENT OF EXISTING ALLOY 617 DATA FOR GEN IV NUCLEAR REACTOR APPLICATIONS

\begin{tabular}{|l|l|l|l|}
\hline Data ID: & Schubert84F6 & Data Class: & 5 \\
\hline Data Source: & German HTGR Project & Reference: & Schubert 1984 \\
\hline Comment: Creep in various environments \\
\hline
\end{tabular}

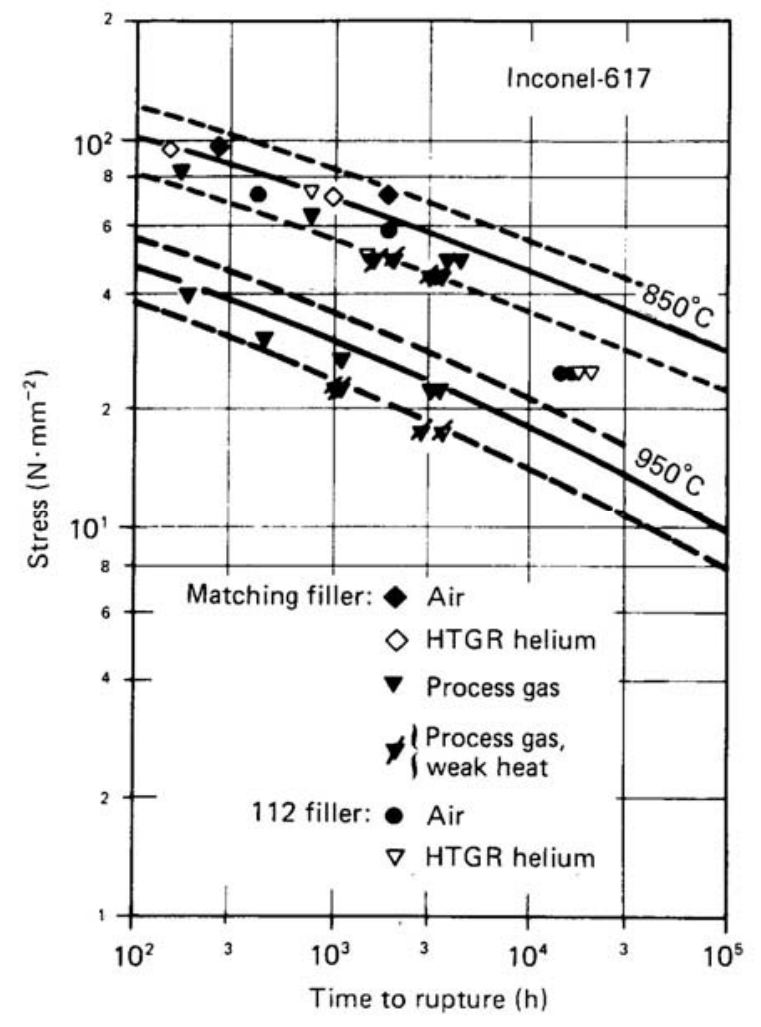

\begin{tabular}{|l|l|l|l|}
\hline Data ID: & Schubert84F8 & Data Class: & 5 \\
\hline Data Source: & German HTGR Project & Reference: & Schubert 1984 \\
\hline Comment: Creep in air and helium & \\
\hline
\end{tabular}

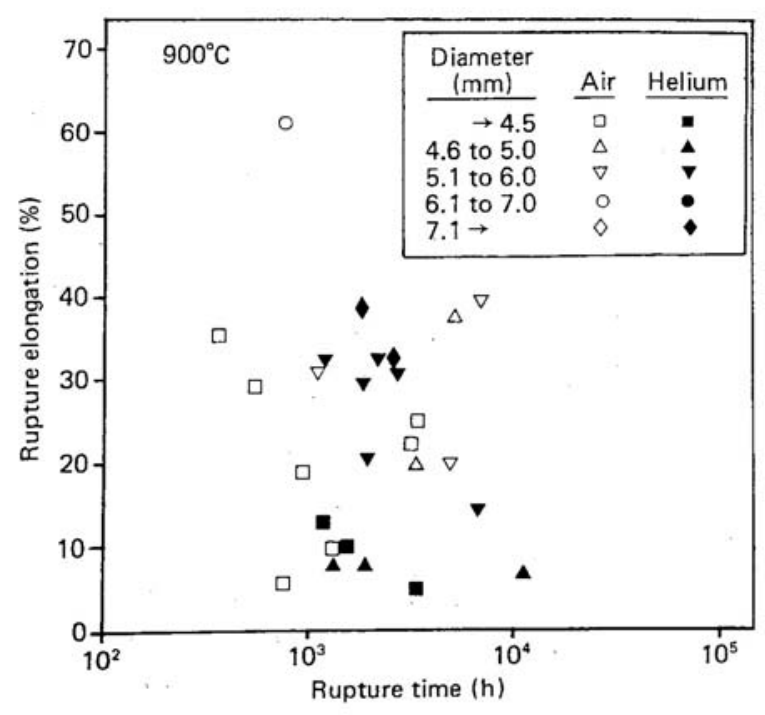


ASSESSMENT OF EXISTING ALLOY 617 DATA FOR GEN IV NUCLEAR REACTOR APPLICATIONS

\begin{tabular}{|l|l|l|l|}
\hline Data ID: & Schubert84F10 & Data Class: & 5 \\
\hline Data Source: & German HTGR Project & Reference: & Schubert 1984 \\
\hline Comment: & \multicolumn{2}{|l|}{} \\
\hline
\end{tabular}

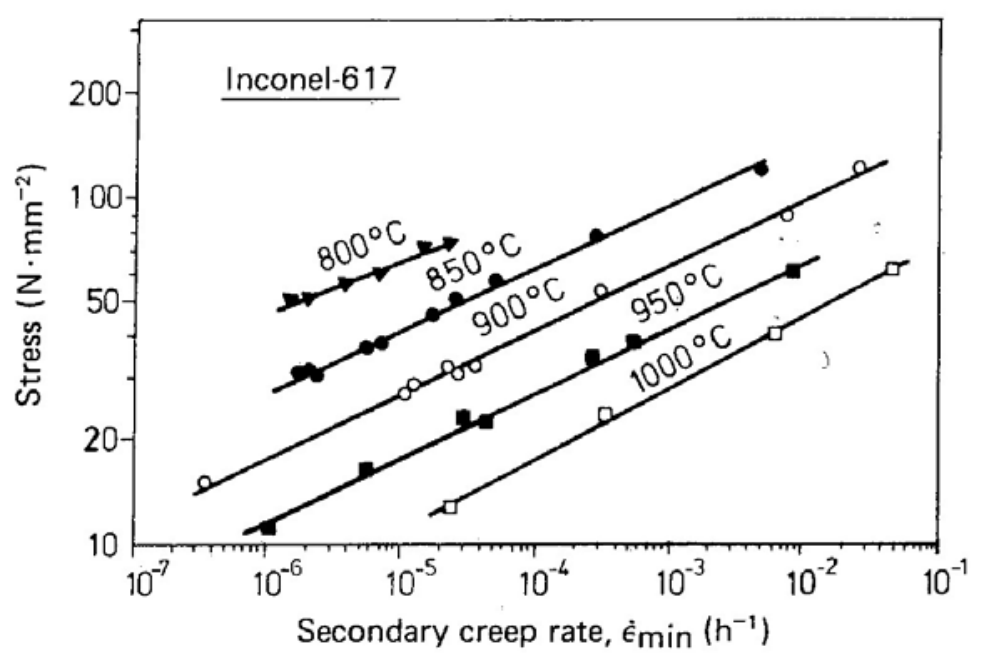




\subsection{Major Data Sources in Acquisition}

\section{$\underline{\text { GE-HTGR Reports }}$}

The source document on the GE-HTGR data assessed in Section 3.1 is only one volume of a series of reports developed by General Electric for the Advanced Gas Cooled Nuclear Reactor Materials Evaluation and Development Program [GCRME\&DP] under contract with the Department of Energy during the 1970's to 1980's. A copy of the report series has been located at Technical Insights (TI), a company in San Diego, California. Negotiations were conducted and have reached an agreement that the company will duplicate the series for ORNL at a reasonable service cost. The acquisition is currently in progress. This series consists of 55 progress reports, topical reports, and final reports published from June 30, 1976 to July 1, 1989 on materials of interest to the Gen IV Materials Handbook including Alloy 617. Because the GE-HTGR data document assessed in Section 3.1 is a summary of GE's efforts on creep and fatigue of Alloy 617 over the eight years from 1978 to 1986, a significant amount of additional processed data may not be expected from the TI duplicates. However, if progress reports on Alloy 617 are included in the duplicates, detailed and less processed data such as creep curves, fatigue loops etc., which are of great interest to the High Temperature Design Methodology (HTDM) development, may be included. Data of other material properties may also be discovered in the duplicates. Furthermore, because some of the GE facility components used in the HTGR materials testing were shipped to ORNL after the program was terminated, detailed testing procedures and facilities design descriptions may still provide valuable information for refurbishing the ORNL testing facilities for the Gen IV Materials Program.

\section{Petten Data}

The German HTGR data reviewed in Section 3.1 were generated during the same period of time when the ORNL-HTGR and GE-HTGR data were generated. After the termination of the U. S. HTGR program around the end of the 1980's much more applicable data on Alloy 617 continued to be generated in Germany [Schubert 1993].

Under the leadership of European Commission Joint Research Centre (JRC), a data network called "Online Data \& Information Network" (ODIN) has been developed for the European energy research community. The ODIN contains engineering databases, document management sites and other information related to European research in the area of nuclear and conventional energy. The scientific and technical responsibilities for the engineering, nuclear and document databases and its administration reside at the Institute for Energy (IE-Petten, located in Petten, Netherlands) in the Data Management $\&$ Dissemination sector. Initially the material properties data were collected into two databases, Alloys-DB and Corrosion-DB respectively. The Alloys-DB covered mechanical and thermo-physical properties for engineering alloys at low and elevated temperatures for base materials and joints. It also included irradiation materials testing in the field of fusion and fission, tests on thermal barrier coating for gas turbines and 
mechanical properties testing on a corroded specimen. The Corrosion-DB contained weight gain/loss data of high temperature exposed engineering alloys, ceramics and hot isostatic pressed (HIP) powder materials and covered corrosion tests such as oxidation, sulfidation and nitridation. The two databases were later merged into a single database named AllCor-DB to include both Alloys (All-) and Corrosion (Cor-) data, and recently the AllCor-DB was renamed as Mat-DB.

The Mat-DB is designed to contain experimental data delivered by laboratories in defined formats and quality. In total, the database for base materials contains more than 130 tables and 1850 fields, which are grouped into several logical entities: data source, material, specimen, and test condition, as summarized in Table 25. The test results data are divided into three parts: mechanical, thermo-physical, and corrosion, each with tables containing test type specific data. The mechanical part covers 23 types of mechanical properties data such as uniaxial tensile, multiaxila tensile, unicaxial creep etc.; the thermo-physical part holds 10 types of thermo-physical properties data such as density, specific heat, etc.; and the corrosion part contains high temperature corrosion data. The extension to other types of corrosion is also under consideration by the management. A summary of the Mat-DB test result data is given in Table 26.

Table 25: A summary of Mat-DB entities

\begin{tabular}{|l|l|}
\hline \multicolumn{1}{|c|}{ Entity } & \multicolumn{1}{c|}{ Content } \\
\hline Data Source & Organization, laboratory, scientist, R\&D project \\
\hline Material & $\begin{array}{l}\text { Material characterization, chemical composition, heat treatment, } \\
\text { process data, microstructure }\end{array}$ \\
\hline Specimen & Sampling, orientation, geometry, coating layers \\
\hline Test condition & Test environment, mechanical or thermal pre-exposure, irradiation \\
\hline Joining & Process method, joining parameters, joining geometry, filler metal \\
\hline Test result & See Table 26 \\
\hline
\end{tabular}

The data of Mat-DB are commercialized information. Properties of Alloy 617 are included in the Mat-DB together with those of Hastelloy X, Nimonic 86 and Alloy $800 \mathrm{H}$ etc. as a package designated as JZ Jülich HTR Data Sets at a sales price of $€ 7000$. $(€ 1.00$ $=\$ 1.22159$ on 10 June, 2005 when this is being written. Introduction for the Mat-DB indicates that the data on Alloy 617 were generated from the German HTGR project. A summary of the Mat-DB data on Alloy 617 is given in Table 27. Communication is underway with the ODIN administration to gain more detailed information about the database and to negotiate the purchase of desired data. The negotiation will include issues such as price and user license agreement, i. e., permission for the purchased data to be collected into the Gen IV Materials Handbook and used by future reactor designers. The purchase will not be realized until it is economically and technically justified. Approximately $\$ 8550$ is needed for the FZ Jülich HTR Data Sets. Preliminary responses from the ODIN administration indicate that the "Test Results" entry in Table 27 means 
the actual number of tests. This means there are many more data points than those test numbers in the Mat-DB. For example, one tensile test can generate several tensile property data points including yield stress, ultimate tensile stress, yield strain, etc. Compared to the cost to experimentally generate the same amount of data, it appears cost effective to purchase the Petten data, especially since the package also includes a similar amount of data for Alloy $800 \mathrm{H}$, another candidate material for Gen IV nuclear reactors. Another advantage of purchasing the data is that the original data that were used to produce the plots in the German HTGR data source document [Schubert 1984] can be obtained.

Table 26: Mat-DB test result entity

\begin{tabular}{|l|l|}
\hline MECHANICAL PROPERTIES & Irradiation \\
\hline Crack Growth \& Fracture & Irradiation creep \\
\hline Creep crack growth & Swelling \\
\hline Cyclic creep crack growth & Tensile \\
\hline Fatigue crack growth & Compression \\
\hline Fracture toughness & Multiaxial tensile \\
\hline Impact & Uniaxial tensile \\
\hline Creep & Small punch tensile \\
\hline Cyclic creep & THERMO-PHYSICAL PROPERTIES \\
\hline Multiaxial creep & Density \\
\hline Torsional creep & Electrical resistivity \\
\hline Uniaxial creep & Emissivity \\
\hline Small punch creep & Linear thermal expansion \\
\hline Relaxation & Poisson's ratio \\
\hline Multiaxial relaxation & Specific heat \\
\hline Uniaxial relaxation & Shear modulus \\
\hline Fatigue & Thermal conductivity \\
\hline High cycle fatigue & Thermal diffusivity \\
\hline Low cycle fatigue (load control) & Young's modulus \\
\hline Low cycle fatigue (strain control) & CORROSION \\
\hline Thermal fatigue & High temperature corrosion \\
\hline Thermo-mechanical fatigue & \\
\hline
\end{tabular}


ASSESSMENT OF EXISTING ALLOY 617 DATA FOR GEN IV NUCLEAR REACTOR APPLICATIONS

Table 27: European mechanical properties data on Alloy 617 in Mat-DB

\begin{tabular}{|c|c|c|c|}
\hline Test Type & Combined Material & Test Results & Temperature ${ }^{\mathbf{C}} \mathbf{C}$ \\
\hline Creep crack growth & & 26 & $800-1000$ \\
\hline Low cycle fatigue (strain control) & & 261 & $20-950$ \\
\hline \multirow{3}{*}{ Uniaxial creep } & & 1134 & $550-1000$ \\
\cline { 2 - 4 } & similar joint & 152 & $600-1000$ \\
\cline { 2 - 4 } & irradiated & 175 & $550-1000$ \\
\hline \multirow{3}{*}{ Uniaxial tensile } & & 141 & $20-1000$ \\
\cline { 2 - 4 } & irradiated & 109 & $20-1000$ \\
\cline { 2 - 4 } & irradiated, similar joint & 26 & $20-1000$ \\
\cline { 2 - 4 } & service exposed & 2 & $20-20$ \\
\hline
\end{tabular}




\section{OTHER DATA SOURCES AND FUTURE WORK}

As previously mentioned in Section 1.2, a title search on Alloy 617 through the CSA Materials Research Database with METADEX has yielded more than 100 documents and a key word search has resulted in more than 700 . To efficiently conduct the existing data assessment within the given timeframe and manpower, the principle of $80 / 20$ must be applied in combination with the priority assessment basis. Once the most important existing data sources are identified, 20 percent of the time spent may complete the assessment of 80 percent of the existing data that are really germane to the Gen IV nuclear reactor materials needs. The remaining data will be assessed on an "as necessary" basis as long as time allows. To date, the priorities have been identified mainly based on the relevancy of the data sources to the intended nuclear application as well as the amount of data contained. The identified high priority data sources have been evaluated or are in the acquisition process as described in the previous two sections. These data sources are believed to cover a fairly large portion of the existing data relevant to Gen IV materials needs for Alloy 617. For future assessment activities, the priority will be identified mainly based on design and qualification requirements for specific property types such as low cycle fatigue, creep crack growth, biaxial fatigue etc. To facilitate this effort and for the purpose of complete documentation, documents that may contain applicable data on Alloy 617 are also listed in the References Section.

It should be pointed out that because most of the existing data were generated from heats that complied with the ASTM standard specifications, which offer considerable allowances for parameters such as chemical composition range, grain size etc., significant data scatter in mechanical properties have been observed. A task has been underway to refine the standard specifications of the alloy in an effort to develop a nuclear application specification with reduced mechanical properties data scatter and improved high temperature properties [Ren 2005]. If that effort is successful, the applicability of the existing data for design and analysis must then be re-evaluated. 


\section{REFERENCES AND DATA SOURCES}

Abd El-Azim, M E; Mohamed, K E; Hammad, F H, "The Deformation Characteristics of Alloy $800 \mathrm{H}$ and Alloy 617", Mechanics of Materials. Vol. 14, No. 1, pp. 33-46. Nov. 1992.

Ali, M K; Hashmi, M S J; Yilbas, B S, “Fatigue Properties of the Refurbished INCO-617 Alloy”, Journal of Materials Processing Technology. Vol. 118, No. 1-3, pp. 45-49. 3 Dec. 2001.

An, S U, " Primary Creep and Microstructure of Inconel 617 at $1073 \mathrm{~K}$ ", Journal of the Korean Institute of Metals and Materials. Vol. 30, No. 3, pp. 247-255. Mar. 1992.

ASME Task Force Very High-Temperature Design, "Draft Alloy 617 Code Case", Cases of ASM Boiler and Pressure Vessel Code, July 27, 1988.

Baldwin, D H; Kimball, O F; Williams, R A, "Design Data for Reference Alloys: Inconel 617 and Alloy 800H", Prepared by General Electric Company for the Department of Energy, Contract DE-AC03-80ET34034, April 1986.

Bassford, T. H., Inconel Alloy 617, Huntington Alloys, Inc., Hundngton, WV, June 1982.

Bassford, T H; Schill, T V, “A Review of Inconel Alloy 617 and Its Properties After Long-Time Exposure to Intermediate Temperatures", Applications of Materials for Pressure Vessels and Piping. MPC-10; San Francisco, Calif ; U.S.A ; June 1979. pp. 112. 1979.

Bruch, U; Schuhmacher, D, "Tensile and Impact Properties of Candidate Alloys for High-Temperature Gas-Cooled Reactor Applications", Nuclear Technology, Vol. 66, August, 1984.

Burke, M A; Beck, C G, "The High-Temperature Low-Cycle Fatigue Behavior of the Nickel-Base Alloy IN-617”, Metall. Trans. A. Vol. 15A, No. 4, pp. 661-670. Apr. 1984.

Cook, R H, "Creep Properties of Inconel-617 in Air and Helium at 800 to $1000^{\circ} \mathrm{C}$ ", Nuclear Technology. Vol. 66, No. 2, pp. 283-288. Aug. 1984.

Cook, R H, "Creep Properties of Inconel 617 in Air and Helium at 800 to $1000^{\circ} \mathrm{C}$ ", Nuclear Technology, 66, 283, 1984.

Corum, J M and Blass, J J, "Rules For Design of Alloy 617 Nuclear Components to Very High Temperatures" pp. 147-153, PVP - Vol. 215, Fatigue, Fracture, and Risk, Am. Soc. of Mechanical Engineers, 1991. 
ASSESSMENT OF EXISTING ALLOY 617 DATA

FOR GEN IV NUCLEAR REACTOR APPLICATIONS

Czyrska-Filemonowicz, A; Ennis, P; Schuster, H; Nickel, H, "Microstructural Evolution of Inconel Alloy 617 During Creep Deformation in Air and in Decarburization Environment", Prace Komisji Metalurgiczno-Odlewniczej, Polska Akademia Nauk-Oddzial W Krakowie, Metalurgia , No. 39, pp. 71-93. 1990.

Ennis, P J; Mohr, K; Schuster, H, "Effect of Carburizing Service Environments on the Mechanical Properties of High Temperature Alloys", Nuclear Technology, Vol. 66, August, 1984.

Ennis, P J; Quadakkers, W J; Schuster, H, "Effect of Selective Oxidation of Chromium on Creep Strength of Alloy 617", Materials Science and Technology. Vol. 8, No. 1, pp. 78-82. Jan. 1992.

Ganesan, P; Smith, G D; Yates, D H, "Performance of Inconel Alloy 617 in Actual and Simulated Gas Turbine Environments", Materials and Manufacturing Processes. Vol. 10, No. 5, pp. 925-938. Sept. 1995.

Gontareva, R G; Kozyrskii; O I, Tikhonov, L V, "Structural Changes in Alloy EI 617 During Thermal Fatigue", Akad. Nauk Ukr. SSR, Metallofiz, No. 70, pp. 52-56. 1977.

Han, Y H; Dzo, M H, "The Actual True Stress Change Due to Pore Formation in Inconel 617 Creep Test”, J. Korean Inst. Met. Vol. 23, No. 5, pp. 489-494. May 1985.

Hattori, H; Kitagawa, M; Ohtomo, A, "Effect of Grain Size on High Temperature LowCycle Fatigue Properties of Inconel 617", Tetsu-to-Hagane (Journal of the Iron and Steel Institute of Japan). Vol. 68, No. 16, pp. 2521-2520. Dec. 1982.

Hattori, H; Kitagawa, M; Ohtomo, A, "Effect of Vacuum Environment on High Temperature Low-Cycle Fatigue Properties of Inconel 617", Journal of the Society of Materials Science, Japan. Vol. 32, No. 357, pp. 667-671. June 1983.

Hattori, H; Kitagawa, M; Ohtomo, A, “An Evaluation of Creep--Fatigue/Environment Behaviors of Inconel 617 And Hastelloy XR for HTGR Application", International Conference on Creep; Tokyo; Japan; 14-18 Apr. 1986. pp. 117-122. 1986.

Hattori, H; Kitagawa, M; Ohtomo, A, "Effect of Specimen Geometry on Low-Cycle Fatigue Property of Inconel 617 at HTGR Temperatures", Journal of the Society of Materials Science, Japan. Vol. 35, No. 391, pp. 343-349. Apr. 1986.

Hosier, J C; Tillack, D J, "Inconel Alloy 617-A New High-Temperature Alloy", Materials Engineering Quarterly, August, 1992.

Hosoi, Y; Abe, S, "Effect of Helium Environment on the Creep Rupture Properties of Inconel 617 at $1000^{\circ} \mathrm{C} "$, Metall. Trans. A. Vol. 6A, No. 6, pp. 1171-1178. June 1975. 
ASSESSMENT OF EXISTING ALLOY 617 DATA

FOR GEN IV NUCLEAR REACTOR APPLICATIONS

Hsu, S S, “Time-Dependent Crack Growth in a Heat-Resistant Alloy Inconel 617”, Journal of Nuclear Science and Technology (Japan). Vol. 30, No. 4, pp. 302-313. Apr. 1993.

Kewther, M A; Hashmi, M S J; Yilbas, B S, “Tensile and Fatigue Testing of Inconel 617 Alloy After Heat Treatment and Electrochemical Tests", Industrial Lubrication and Tribology. Vol. 53, No. 3, pp. 112-118. May-June 2001.

Khasin, G A; Brazgin, I A; Ermanovich, N A; Eremeev, V I; Tarnovsky, V I, "Resistance to Deformation of High-Temperature Alloys EI 617 and EI 427B, and Steel EI481", IZVEST VUZ Chernaya Met, --7--, 101-105, 1969.

Kihara, S; Newkirk, J B; Ohtomo, A; Saiga, Y, "Morphological Changes of Carbides During Creep and Their Effects on the Creep Properties of Inconel 617 at $1000^{\circ} \mathrm{C}^{\prime}$, Metall. Trans. A. Vol. 11A, No. 6, pp. 1019-1031. June 1980.

Kimball, O F; Lai, G Y; Reynolds, G H, "Effects of Thermal Aging on the Microstructure and Mechanical Properties of a Commercial Ni-Cr-Co-Mo Alloy (Inconel 617) ", Metall. Trans. A. Vol. 7A, No. 12, pp. 1951-1952. Dec. 1976.

Kitagawa, M; Tamura, K; Ohtomo, A, "Fracture Criterion of Inconel 617 Under Cyclic Straining at High Temperatures", Journal of the Society of Materials Science, Japan. Vol. 32, No. 357, pp. 662-666. June 1983.

Kozyrskii, O I; Petrunin, G A; Tikhonov, L V, "Effect of the Conditions of Preliminary Heat Treatment on the Structure and Properties of the Alloy EI 617 During Creep", Probl. Prochn, No. 1, pp. 39-43. Jan. 1976.

Krompholz, K; Grosser, E D; Ewert, K, "Determination of J-Integral R-Curves for Hastelloy X and Inconel 617 up to 1223 Degree K Using the Potential Drop Technique", Z. Werkstofftech. Vol. 13, no. 7, pp. 236-244. July 1982.

Kufaev, V N; Ishchenko, I I; Pogrebnyak, A D; Sinaisky, B N, "Effect of the Unsteady State of The Loading on the Fatigue Life and Strength of the Alloy EI617 at High Temperature", FIZ-KHIM MEKHAN MAT. Vol. 5, No. 2, pp. 142-145. Mar.-Apr. 1969.

Laanemae, W M; Bothe, K; Gerold, V, "High Temperature Mechanical Behaviour of Alloy 617. II. Lifetime and Damage Mechanisms", Zeitschrift fur Metallkunde. Vol. 80, No. 12, pp. 847-857. Dec. 1989.

Laanemae, W M; Bothe, K; Gerold, V, "High-Temperature Mechanical Behaviour of Alloy 617. Deformation Mechanisms", Zeitschrift fur Metallkunde. Vol. 80, No. 11, pp. 788-799. Nov. 1989.

Laanemaee, W M, "Damage of Nickel Base Alloy 617 in the Case of Creep and High Temperature Change Deformation”, pp. 196, 14 Feb. 1989. 
ASSESSMENT OF EXISTING ALLOY 617 DATA

FOR GEN IV NUCLEAR REACTOR APPLICATIONS

Lipscomb, W G, "Mechanical Properties and Corrosion Resistance of Inconel Alloy 617 for Refinery Service", W.G. Lipscomb, et. al., Corrosion 89/259, NACE, Houston, TX, 1989.

Mankins, W L; Hosier J C; Bassford, T H, "Microstructure and Phase Stability of INCONEL Alloy 617”, Metallurgical Transactions, Vol. 5, December 1974.

Mannan, S K; Smith, G D; Wilson, R K, "Effect of Grain Size, Microstructure, Test Temperature, and Frequency on the Low Cycle Fatigue Properties of Inconel Alloy 617", Structure-Property Relationships and Correlations With the Environmental Degradation of Engineering Materials; Monterey, California; United States; 31 July-1 Aug. 1991. pp. 387-428. 1992.

McCoy, H E; "Mechanical Properties Of Hastelloy X and Inconel 617 After Aging 53,000 Hours in HTGR-He”, Oak Ridge National Laboratory, ORNL/TM-9604, 1985.

McCoy, H E; King, J F; "Mechanical Properties of Inconel 617 and 618”, Oak Ridge National Laboratory, ORNL/TM-9337, 1985.

Meurer, H P; Gnirss, G K H; Mergler W; Raule G; Schuster H; Ullrich G; "Investigations on the Fatigue Behavior of High-Temperature Gas-Cooled Reactor Components", Nuclear Technology, Vol. 66, August 1984.

Meurer, H P; Breitling, H; Dietz, W, "Influence of Hold-Time and Strain Rate on the LCF Behaviour of Alloy 617 at $950^{\circ} \mathrm{C}^{\prime}$, Low Cycle Fatigue and Elasto-Plastic Behaviour of Materials; Munich; FRG; 7-11 Sept. 1987. pp. 246-251. 1987.

Meurer, H P; Hanswillemenke, H; Breitling, H; Dietz, W, "Biaxial Fatigue Tests on Thin Walled Tubes of NiCr23Co12Mo (Inconel 617) at $950^{\circ} \mathrm{C}$. (Retroactive Coverage)", Fatigue Under Biaxial and Multiaxial Loading; Stuttgart; Germany; 3-6 Apr. 1989. pp. 249-264. 1991.

Meyer-Olbersleben, F; Meyer-Olbersleben, F; Kasik, N; Ilschner, B, " The Thermal Fatigue Behavior of the Combustor Alloys IN 617 and Haynes 230 Before and After Welding", Metallurgical and Materials Transactions A. Vol. 30A, No. 4, pp. 981-989A. Apr. 1999.

Mino, K; Ohtomo, A; Saiga, Y, "Effect of Grain Boundary Migration and Recrystallization on the Creep Strength of Inconel 617”, Tetsu-to-Hagane. Vol. 63, No. 14, pp. 2372-2380. Dec. 1977.

Mino, K; Ohtomo, A, "Effect of Grain Boundary Migration and Recrystallization on the Creep Strength of Inconel 617”, Trans. Iron Steel Inst. Jpn. Vol. 18, No. 12, pp. 731-738. 1978. 
ASSESSMENT OF EXISTING ALLOY 617 DATA

FOR GEN IV NUCLEAR REACTOR APPLICATIONS

Mino, K; Kitagawa, M; Ohtomo, A; Saiga, Y, "Effect of Thermal and Mechanical History on the Creep Rate of Inconel 617', Trans. Iron Steel Inst Jpn. Vol. 20, No. 12, pp. 826-832. 1980.

Mino, K; Kitagawa, M; Ohtomo, A; Fukagawa, M, “Creep Rupture Properties of Inconel 617 in a Simulated HTR [High Temperature Gas-Cooled Reactor]-Helium Atmosphere", Tetsu-to-Hagane (J. Iron Steel Inst. Jpn.). Vol. 68, No. 3, pp. 477-485. Mar. 1982.

Mishin, E. V; Loginov, N Z; Shkanov, I N, "The Effect of Stress Concentrations and Surface Hardening on the Fatigue Strength of the Alloy EI 617", TRUDY KAZANSK AVIATS INST, --102--, 31-36, 1968.

U. S. DOE Nuclear Energy Research Advisory Committee and the Generation IV International Forum, "A Technology Roadmap for Generation IV Nuclear Energy Systems”, GIF-002-00, December, 2002.

Ohnami, M; Imamura, R, "Effect of Vacuum Environment on Creep Rupture Properties of Inconel 617 at $1000^{\circ} \mathrm{C}$ (Especially Based On Crack Initiation And Propagation) ", Bull. Jpn. Soc. Mech. Eng. Vol. 24, No. 95, pp. 1530-1536. Sept. 1981.

Osthoff, W; Schuster, H; Ennis, P J; Nickel, H, "Creep and Relaxation Behavior of Inconel-617”, Nuclear Technology. Vol. 66, No. 2, pp. 296-307. Aug. 1984.

Osthoff, W; Schuster, H; Ennis, P J; Nickel, H, "The Creep and Relaxation Behavior of Inconel 617', Creep and Fracture of Engineering Materials and Structures, Vol. 1; Swansea; U.K ; 1-6 Apr. 1984. pp. 307-318. 1984.

Osthoff, W; Schuster, H and Ennis, P, "Creep and Relaxation Behavior of Inconel 617", Nuclear Technology, 66, 383, 1984.

Penkalla, H J; Over, H H; Schubert, F; Nickel, H, "Design Values of Inconel 617 for High Temperature Reactors Operating at Temperatures Above $800^{\circ} \mathrm{C}$ ", Nuclear Engineering and Design. Vol. 83, No. 3, pp. 397-402. Dec. (II) 1984.

Penkalla, H J; Schubert, F; Nickel, H, "Torsional Creep of Alloy 617 Tubes at High Temperature", Superalloys 1988; Champion, Pennsylvania; USA; 18-22 Sept. 1988. pp. 643-652. 1988.

Penkalla, H J; Nickel, H; Schubert, F, "Multiaxial Creep of Tubes from Incoloy 800H and Inconel 617 Under Static and Cyclic Loading Conditions", Nuclear Engineering and Design. Vol. 112, pp. 279-289. Mar. 1989.

Penkalla, HJ; Schubert, Florian; Nickel, Hubertus, "Description of Creep and Fatigue Exposed Tubes of Alloy 617", ASME Pressure Vessels Piping Division Publication, PVP, ASME, New York, NY, (USA), Vol. 262, pp. 221-224, 1993. 
ASSESSMENT OF EXISTING ALLOY 617 DATA

FOR GEN IV NUCLEAR REACTOR APPLICATIONS

Rao, K B S; Schiffers, H; Schuster, H, "Low Cycle Fatigue Behaviour of Inconel Alloy 617", High Temperature Alloys--Their Exploitable Potential; Petten; The Netherlands; 15-17 Oct. 1985. pp. 411-422. 1987.

Rao, K B S; Meurer, H P; Schuster, H, "Creep--Fatigue Interaction of Inconel 617 at $950^{\circ} \mathrm{C}$ in Simulated Nuclear Reactor Helium", Materials Science and Engineering A. Vol. A104, pp. 37-51. Oct. 1988.

Rao, K B S; Schiffers, H; Schuster, H; Nickel, H, "Influence of Time and Temperature Dependent Processes on Strain Controlled Low Cycle Fatigue Behavior of Alloy 617", Metall. Trans. A. Vol. 19A, No. 2, pp. 359-371. Feb. 1988.

Ren, W. and R. W. Swindeman, "High Temperature Metallic Materials Test Plan for Generation IV Nuclear Reactors", ORNL/TM-2005/507, Revision 1, Oak Ridge National Laboratory, U. S. Department of Energy Generation IV Nuclear Reactor Program, November 30, 2004.

Ren, W. and Swindeman, R. W., "Development of a Controlled Material Specification for Alloy 617 for Nuclear Applications", Oak Ridge National Laboratory, ORNL/TM2005/504, U. S. Department of Energy Generation IV Nuclear Reactor Program, May 30, 2005.

Richter, F, "Thermophysical Properties of the High Temperature Material NiCr22Co12Mo (Inconel 617)", Materialwissenschaft und Werkstofftechnik. Vol. 19, No. 2, pp. 55-61. Feb. 1988.

Rittenhouse, P. and Ren., W., "Gen IV Materials Handbook Implementation Plan”, Oak Ridge National Laboratory, ORNL/TM-2005/77, U. S. Department of Energy Generation IV Nuclear Reactor Program, March 29, 2005.

Rodig, M; Huthmann, H; Hartnagel, W, "Creep and Fatigue Crack Growth of the Materials X10Ni CrAlTi32 20 and NiCr22Co12Mo. (Alloy 800 And Inconel 617)", Modern Materials (Moderne Werkstoffe); Nurnberg; Germany; 20-21 Mar. 1990. pp. 245-256. 1990.

Rodig, M; Huthmann, H; Hartnagel, W, "Fatigue and Creep Crack Growth of Alloy 800 and Alloy 617 at High Temperatures", Materials at High Temperatures. Vol. 10, No. 4, pp. 268-274. Nov. 1992.

Schneider, K; Ilschner, B, "Creep Behavior of Materials for High-Temperature Reactor Application”, Nuclear Technology, Vol. 66, August 1984.

Schubert, F; Penkalla, H J and Ull-Rich, G, "Creep-Rupture Behaviour, A Criterion for the De-sign of Metallic HTR Components with High Application Temperatures," Proc. IAEA Specialists' Mtg. High Temperature Metallic Materials for Application in 
ASSESSMENT OF EXISTING ALLOY 617 DATA

FOR GEN IV NUCLEAR REACTOR APPLICATIONS

Gas-Cooled Reactors, Vienna, May 4-6, 1981, OEFZS Report 4086, International Atomic Energy Agency, 1981.

Schubert, F; Bruch, U; Cook, R; Diehl, H; Ennis, P J; Jakobeit, W H; Penkalla, J; Heesen, E T and Ullrich, G, "Creep Rupture Behavior of Candidate Materials for Nuclear Process Heat Applications”, Nuclear Technology, 66, 227, 1984.

Schubert, F; Breitbach, G; Nickel, H, "German Structural Design Rule KTA 3221 for Metallic HTR-Components", High-Temperature Service and Time-Dependent Failure, PVP Vol. 262, 9-18, Am. Soc. of Mechanical Engineers, 1993.

Schwertel, J; Merckling, G; Hornberger, K; Schinke, B; Munz, D, "Experimental Investigations on the Ni-Base Superalloy IN617 and Their Theoretical Description", MDVol. 26/AMD-Vol. 121, High Temperature Constitutive Modeling - Theory and Application, ASME 1991.

Shimikawa, T, "Sophisticated Creep-Fatigue Life Estimation Scheme For Pressure Vessel Components Based on Stress Redistribution Locus Concept" PVP-Vol. 472, Elevated Temperature Design and Analysis, Nonlinear Analysis, and Plastic Components - 2004 July 25-29, 2004, PVP2004-2258.

SIMMONS, W. F. "Compilation of Chemical Com-positions and Creep Rupture Strength of Superalloys," ASTM Data Series Publication No. DS 9 D, American Society for Testing and Materials, 1968.

Srivastava, S K; Klarstrom, D L, "The LCF Behavior of Several Solid Solution Strengthened Alloys Used in Gas Turine Engines", Gas Turbine and Aeroengin Congress and Exposition, Bressels, Belgium, June 11-14, 1990.

Stentz, R H; Neugebauer, R B; Merkle, R A, "Elevated Temperature, Partia-Constraint and Thermal Ratcheting Simulations Using Closed-Loop Testing Machines", PVP-Vol. 262, High-Temperature Service and Time-Dependent Failure, ASME, 1993.

Strizak, J. P. et al., "High-Temperature Low-Cycle Fatigue and Tensile Properties of Hastelloy X and Alloy 617 in Air and HTGR Helium", 1981, 15 pp. refs. Presented at IAEA Specialists Meeting on High-Temperature Materials for Application in Gas-Cooled Reactors", Vienna, 4 May 1981, (CONF-810530-4) Avail: NTIS.

Strizak, J. P. et al., "Influence of Temperature, Environment and Thermal Aging on the Continuous Cycle Fatigue Behavior of Hastelloy X and Inconel 617", Oak Ridge National Lab., Tenn. Apr. 1982, 50 p., refs. (ORNL/TM-8130). Avail: NTIS.

Tanabe, T; Sakai, Y; Shikama, T; Fujitsuka, M; Yoshida, H and Watanabe, R, "Creep Rupture Properties of Superalloys Developed for Nuclear Steelmaking", Nuclear Technology, 66, 260, 1984. 
ASSESSMENT OF EXISTING ALLOY 617 DATA

FOR GEN IV NUCLEAR REACTOR APPLICATIONS

Whittenberger, J D, "Tensile Properties of Haynes Alloy 230 and Inconel 617 After Long Exposures to LiF-22 $\mathrm{CaF}_{2}$ and Vacuum At 1093K", Journal of Materials Engineering and Performance. Vol. 3, No. 6, pp. 763-774. Dec. 1994.

Yamamoto, S; Fujiwara, A, "Creep Rupture Strength of Inconel 617 Pipe-to-Pipe Interconnections Subjected to Internal Pressure", Pressure Engineering (Japan) (Atsuryoku Gijitsu-Journal of the High Pressure Institute of Japan). Vol. 20, No. 3, pp. 121-128. Mar. 1982.

Yates, D H; Ganesan, P; Smith, G D, "Recent Advances in the Enhancement of Inconel Alloy 617 Properties to Meet the Needs of the Land Based Gas Turbine Industry", Advanced Materials and Coatings for Combustion Turbines; Pittsburgh, Pennsylvania; United States; 17-21 Oct. 1993. pp. 89-97. 1994.

Yukawa, S. "Re-Analysis of Inconel 617 Fatigue Data" Committee Correspondence, ASME SG-HTD, Sept. 15, 1988.

Yukawa, S. "Elevated Temperature Fatigue Design Curves for Ni-Cr-Co-Mo Alloy 617" JSME/ASME Joint International Conf. on Nuclear Eng., Nov. 4-7, 1991.

Yun, H M; Ennis, P J; Nickel, H; Schuster, H, "The Effect of High-Temperature Reactor Primary Circuit Helium on the Formation and Propagation of Surface Cracks in Alloy $800 \mathrm{H}$ and Inconel 617”, Journal of Nuclear Materials. Vol. 125, No. 3, pp. 258-272. Aug. 1984. 